FERMILAB-Pub-87/125-E

7000.740

\title{
Physics at $\sqrt{ }(\mathrm{s})=2 \mathrm{TeV}$ An Experimentalist's Perspective*
}

\author{
R. Raja \\ Fermi National Accelerator Laboratory \\ P.O. Box 500, Batavia, Illinois 60510
}

April 1987

*Based on lectures delivered at the 2nd school of Particle Physics, Cuernevaca, Mexico, December 4-12, 1986. 


\title{
PHYSICS AT $\sqrt{(s)}=2$ TEV An Experimentalist's perspective'

\author{
Rajendran Raja \\ Fermi National Accelerator Laboratory ${ }^{2}$ \\ Batavia, Illinois, 60510, USA
}

April 1987

\begin{abstract}
This article is based on a series of 5 lectures delivered at the Mexican School. It is intended as an introduction to the physics expected at the Fermilab $\bar{p} p$ collider, the Tevatron, and as such relies heavily on its extrapolative power on physics done at lower energies at the CERN ISR and $s \bar{p} p s$. The lectures were delivered to graduate students and, where possible, I have attempted to give derivations of the basic formulae. The lectures deal with minimum bias physics, QCD jets, W/Z production, Heavy Flavor production and exotic phenomena beyond the standard model and each subject is covered in some detail from the experimentalist's point of view.
\end{abstract}

\footnotetext{
${ }^{1}$ Based on lectures delivered at the 2nd school of Particle Physics, Cuernevaca, Mexico, December 4-12 1986

${ }^{2}$ Fermilab is operated by the Universities Research Association Inc. under contract with the U.S Department of Energy
} 


\section{Minimum Bias Physics}

"Minimum bias" physics derives its name from the characteristics of the event trigger used at the collider to obtain the data. If one were to record an event each time an interaction were to take place, the trigger used would have the least bias and the data recorded would represent most of the total cross section. Only the rare events would be missed. Other terms used to represent minimum bias physics are "Low $p_{t}$ physics" and "bubble chamber physics" at the fixed target.

Minimum bias physics broadly consists of the following subtopics.

- Elastic Scattering

- Inclusive Cross sections, Diffraction dissociation, Central Production

- Multiplicity Distributions, KNO scaling Violations, Minijets

- Quark Gluon plasma

An excellent review of minimum bias physics at the CERN collider is by Rushbrooke [1].

\section{$1.1 \quad$ Elastic Scattering}

The fundamental ideas in the theory of elastic scattering[2] may be understood by considering the problem of scattering between a plane wave $\psi=e^{i k z}$ and an infinitely heavy scattering center(See Figure 1 ). $k=1 / \lambda$ and $2 \pi \lambda$ is the de Broglie wavelength. The wave can be decomposed asymptotically into incoming $\left(e^{-i k r}\right)$ and an outgoing $\left(e^{i k r}\right)$ spherical waves. The expansion is

$$
\psi_{i}=\frac{i}{2 k r} \sum_{l}(2 l+1)\left[(-1)^{l} e^{-i k r}-e^{i k r}\right] P_{l}(\cos \theta)
$$

$P_{l}(\cos \theta)$ are the Legendre polynomials. The scattering process alters the phase and amplitude of the outgoing wave. So the total wave now becomes

$$
\psi_{t o t a l}=\frac{i}{2 k r} \sum_{l}(2 l+1)\left[(-1)^{l} e^{-i k r}-\eta_{l} e^{2 i \delta_{t}} e^{i k r}\right] P_{l}(\cos \theta)
$$




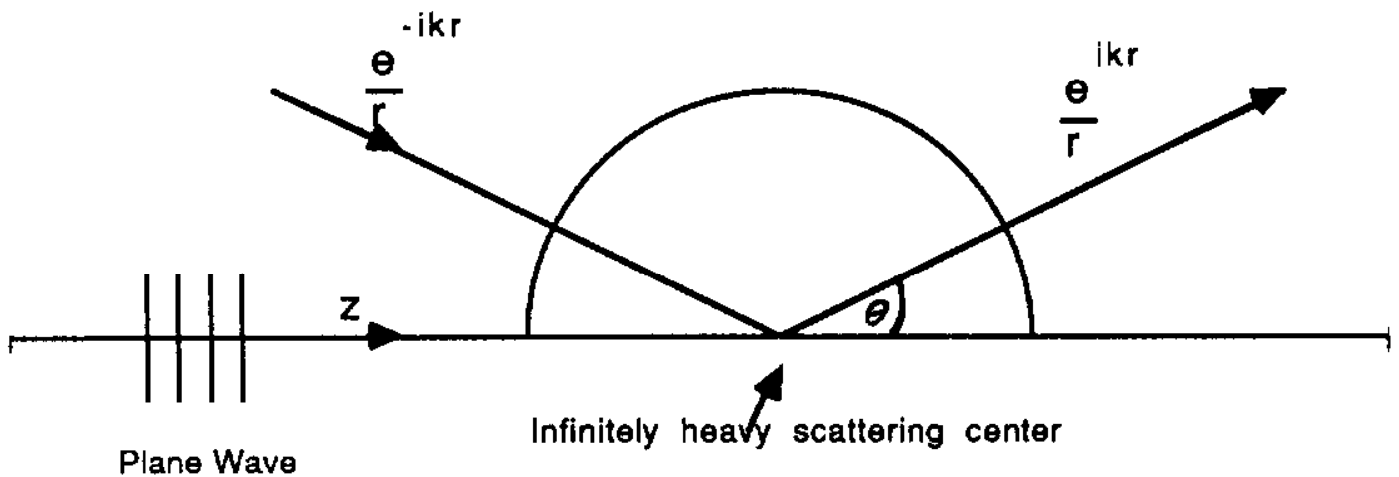

Figure 1: Scattering of a plane wave off an infinitely heavy scattering center

$\eta_{l}$ varies between 0 and 1 and represents the amount the incoming partial wave of angular momentum $l$ is absorbed. The quantity $\delta_{l}$ is known as the phase shift for the $l^{\text {th }}$ partial wave. The scattered wave is the difference between the outgoing and the incoming waves with and without the scattering potential

$$
\psi_{\text {scat. }}=\psi_{\text {total }}-\psi_{i}=\frac{e^{i k r}}{k r} \sum_{l}(2 l+1)\left[\frac{\eta_{l} e^{2 i \delta_{l}}-1}{2 i}\right] P_{l}(\cos \theta)=\frac{e^{i k r}}{r} F(\theta)
$$

The scattered wave clearly corresponds to elastic scattering, since the incoming and outgoing wave numbers are the same. The elastic scattering amplitude $F(\theta)$ is given by

$$
F(\theta)=\frac{1}{k} \sum_{l}(2 l+1)\left[\frac{\eta_{l} e^{2 i \delta_{l}}-1}{2 i}\right] P_{l}(\cos \theta)
$$

It can be easily shown that the elastic differential cross section $\left(\frac{d \sigma}{d \Omega}\right)_{e l}=$ $|F(\theta)|^{2}$. This leads to the elastic cross section

$$
\sigma_{e l}=4 \pi \lambda^{2} \sum_{l}(2 l+1)\left|\frac{\eta_{l} e^{2 i \delta_{l}}-1}{2 i}\right|^{2}
$$


If any $\eta_{l}$ is less than unity, there is absorption of the incoming wave and other processes apart from elastic cross section are present. The reaction cross section $\sigma_{r}$ is then obtained by conserving probability.

$$
\sigma_{r}=\int\left(\left|\psi_{\text {in }}\right|^{2}-\left|\psi_{\text {out }}\right|^{2}\right) r^{2} d \Omega
$$

This leads to

$$
\sigma_{r}=\pi \lambda^{2} \sum_{l}(2 l+1)\left(1-\eta_{l}^{2}\right)
$$

The total cross section is then given by

$$
\sigma_{T}=\sigma_{r}+\sigma_{e l}=\pi \lambda^{2} \sum_{l}(2 l+1) 2\left(1-\eta_{l} \cos 2 \delta_{l}\right)
$$

Using equations (5) and (8) we get,

$$
\operatorname{Im} F(0)=\frac{1}{2 k} \sum_{l}(2 l+1)\left(1-\eta_{l} \cos 2 \delta_{l}\right)
$$

which leads directly to the optical theorem

$$
\operatorname{Im} F(0)=\frac{k}{4 \pi} \sigma_{T}
$$

We see from equation(5) that the maximum elastic cross section for the lth partial wave occurs when $\delta_{l}=\pi / 2$, and $\eta_{l}=1$.

$$
\sigma_{e l}^{\max }=4 \pi \lambda^{2}(2 l+1)
$$

\subsubsection{The black disc model}

Similarly, the reaction cross section attains a maximum value when $\eta_{l}=0$ . i.e there is pure absorption.

$$
\sigma_{r}^{\max }=\pi \lambda^{2} \sum_{l}(2 l+1)
$$

In this limit, known as the black disc limit, the elastic scattering cross section is equal to the reaction cross section and is thus half the total cross 
section, as can be seen from equations (5) and (8). Equation (12) can be understood in a different way. An orbital angular momentum $l$ corresponds to an "impact parameter" b given by $l \hbar=p b$ or $b=l \lambda$. Particles of angular momentum between $l$ and $l+1$ impinge upon and are totally absorbed by an annular ring of cross-sectional area

$$
\sigma=\pi\left(b_{l+1}^{2}-b_{l}^{2}\right)=\pi \lambda^{2}(2 l+1)
$$

Summing up over all $l$ leads to equation (12). This introduces the idea of the impact parameter and the entire partial wave formalism above can be cast into an equivalent impact parameter picture. Also we have assumed a beam of particles being incident on an infinitely massive scattering center. In practice, in the center of mass, the initial state can be described by two plane waves of momenta $k$ and $-k$. The scattering center can be thought of as a potential at the origin. By considering merely the plane wave of one of the incident particles e.g the proton in $\bar{p} p$ scattering, it is possible to convince oneself that the above formalism goes through. When all $\eta_{l}=0$, and there is perfect absorption, $\sum_{l}(2 l+1)=l_{\text {max }}^{2}$. There has to be an $l_{\max }$ due to the finite size of the target. So $\sigma_{e l}$ and $\sigma_{r}$ both become equal to $\pi b_{\text {max }}^{2}$, where $b_{\max }$ is the maximum impact parameter that contributes. This quantity is also sometimes written as the radius $\mathrm{R}$ of the black disc. The differential elastic cross section in the black disc limit can be obtained from equation(4). The sum over the Legendre polynomials can be approximated for small scattering angles, by a Bessel function of the first order. In terms of the momentum transfer $q=2 p \sin (\theta / 2)$ where $\mathrm{p}$ is the center of mass momentum of the colliding particles,

$$
\frac{d \sigma_{e l}}{d q^{2}}=\pi R^{4}\left|\frac{J_{1}(R q)}{R q}\right|^{2} \approx \frac{\pi R^{4}}{4} \exp \left(-\frac{R^{2} q^{2}}{4}\right)
$$

For a typical $\mathrm{R}=1 \mathrm{fm}$, the second expression is valid for $q^{2}<0.2(\mathrm{GeV} / \mathrm{c})^{2}$. For higher values of $\mathrm{Rq}$, the Bessel function undergoes maxima and minima characteristic of diffraction phenomena. The elastic scattering amplitude is purely imaginary in the black disc limit as can be seen from equation (4). The slope of the exponential falloff in $q^{2}$ in the elastic differential cross section is proportional to $R^{2}$. We will denote this quantity B. Figure (2) shows the pp elastic differential cross section at ISR energies([6]). A clear 


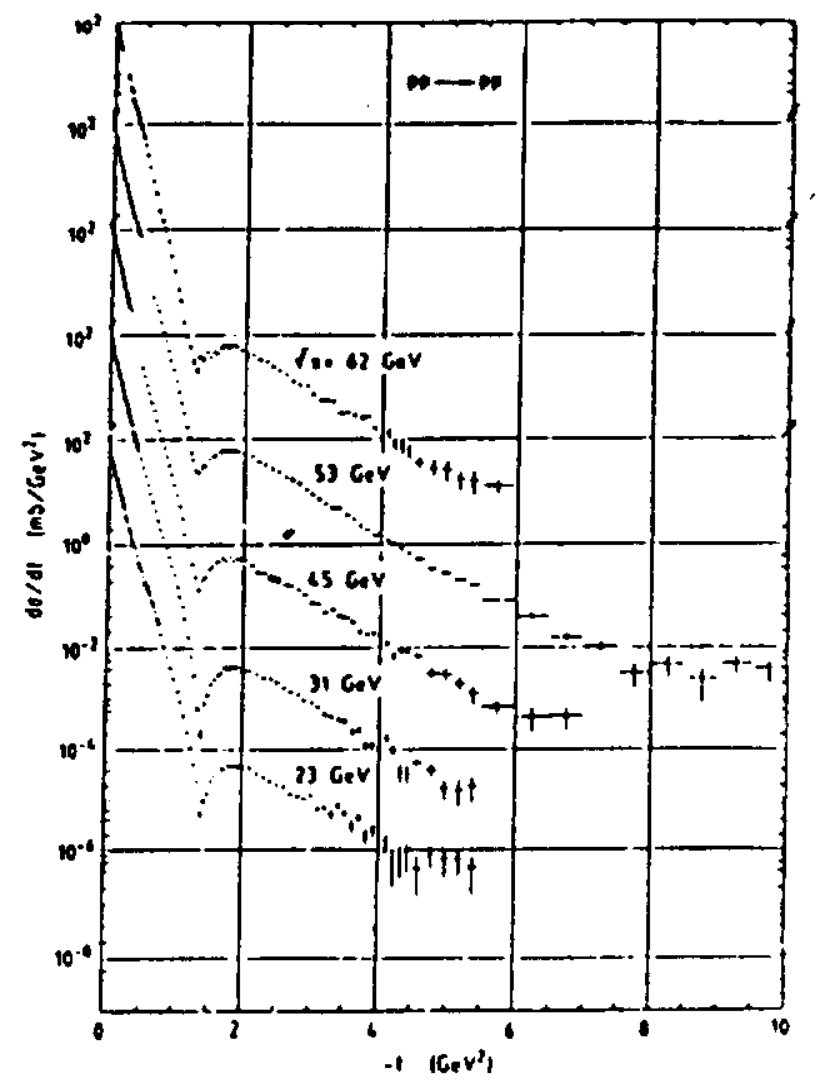

Figure 2: Elastic differential cross section for pp scattering as a function of $-t=q^{2}$ for various ISR energies.

The exponential slope $B$ increases slowly with $\sqrt{(s)}$. This can be seen more explicitly in Figure (3) where a steady increase in $B$ is seen as a function of $\sqrt{(s)}$. This implies that the radius of the proton (antiproton) is increasing with the center of mass energy. This interpretation is further confirmed by the trend of the total cross sections which in this model is also proportional to $R^{2}$. Figure (4) shows that both the $p p$ and the $\bar{p} p$ total cross sections increase with energy roughly as $\ln ^{2}(s)$. Both these trends lend qualitative support to the black disc model, although quantitatively the model fails in the following respects.

- The ratio of $\frac{\sigma_{\text {al }}}{\sigma_{\mathrm{r}}}$ is not 0.5 experimentally.

- The ratio $\rho=\frac{R e(F(0))}{I m(F(0))}$ is not zero as would be expected for perfect absorption.See Figure (5). The curves on this figure are dispersion relation fits to the data $[8]$

- The total cross section is not constant with energy. 


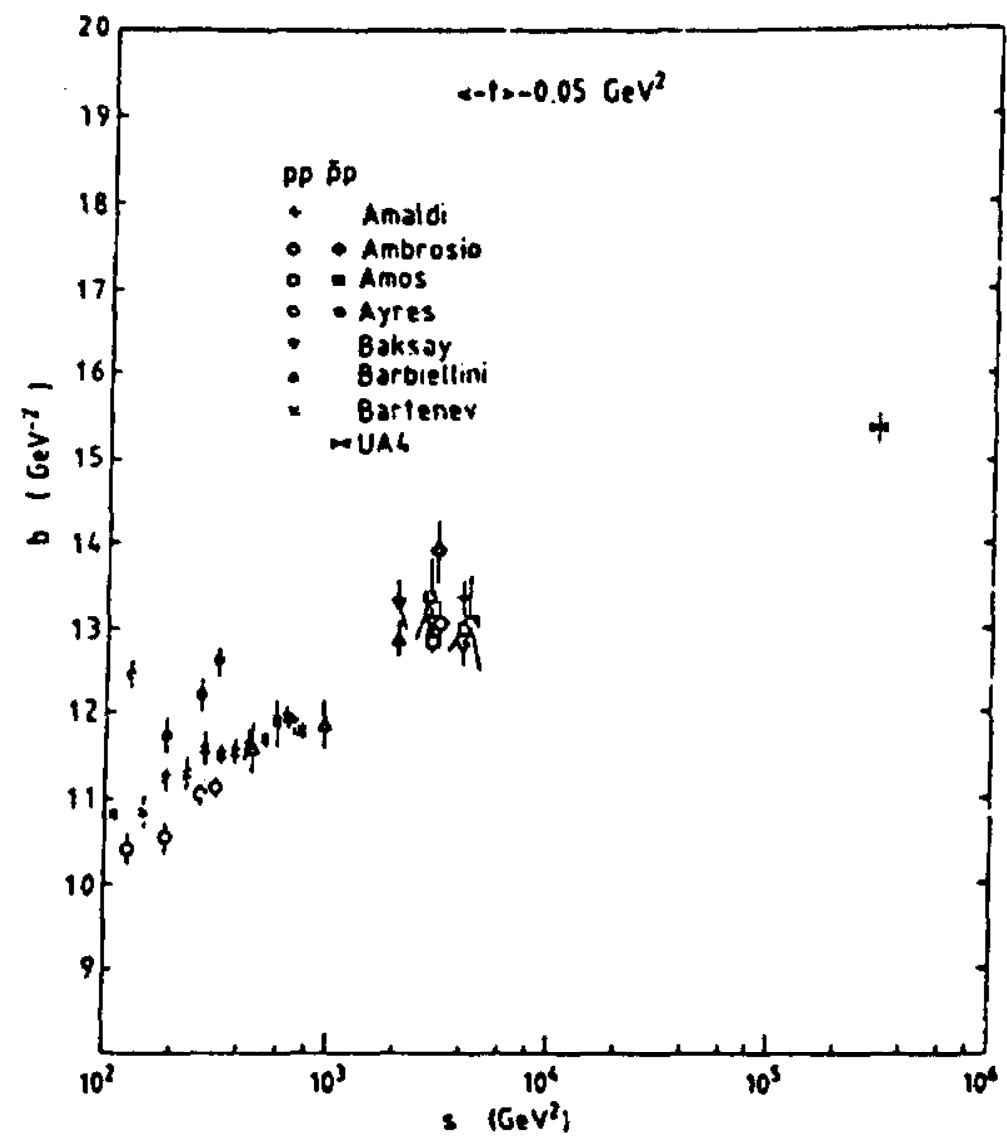

Figure 3: Slope of the elastic differential cross section B as a function of $s$ 


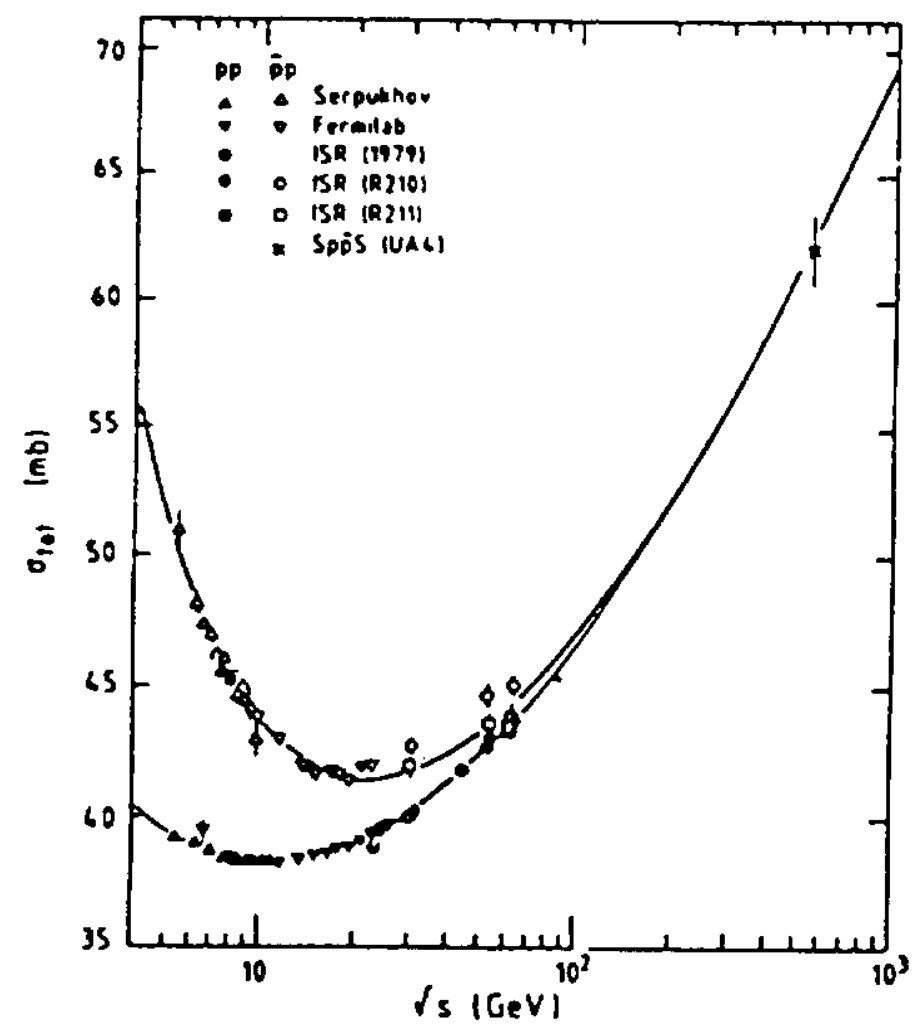

Figure 4: $\bar{p} p$ and $p p$ total cross sections as a function of $s$ 


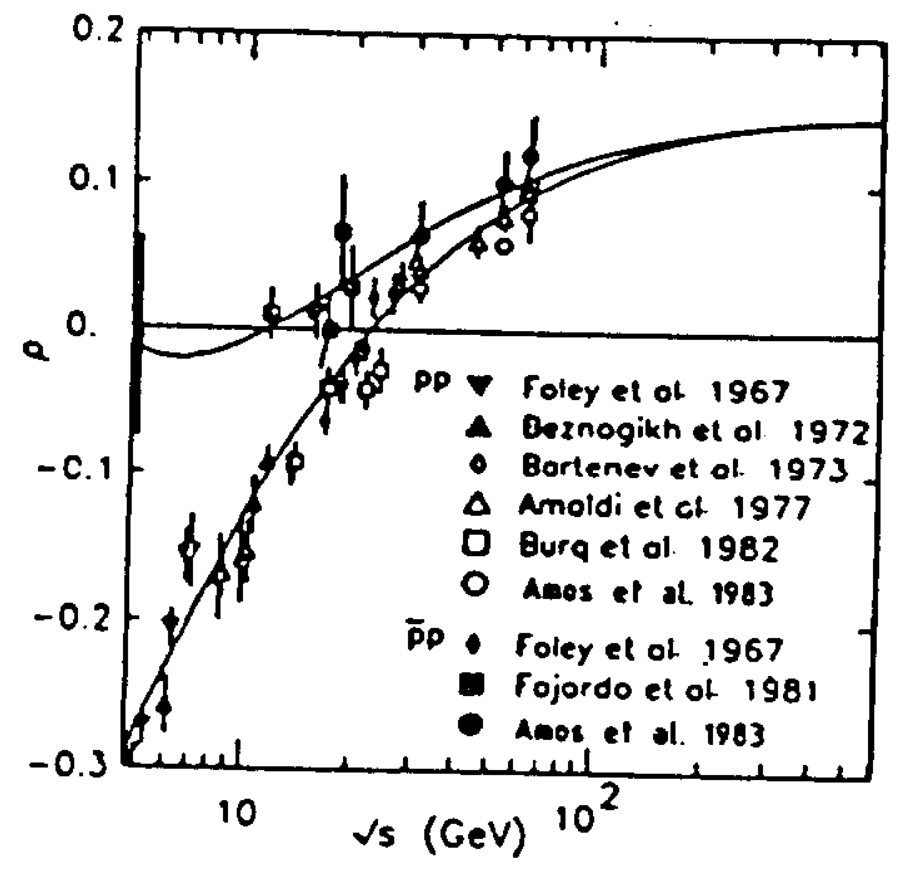

Figure 5: Ratio of the Real part to the imaginary part of the forward elastic scattering amplitude as a function of $s$.

\subsubsection{Geometric Scaling}

These flaws in the simple black disc model can be cured somewhat by the hypothesis of geometric scaling [7] which postulates that the opacity of the black disc, though not perfect, is constant with energy and that the radius $R$ varies as $\ln (s)$. This hypothesis immediately predicts that the total cross section $\sigma_{T}$ goes as $\ln ^{2}(s)$ and that the elastic slope $B$ behaves the same way. Geometric scaling predicts that the quantity $\frac{1}{\sigma_{T}} \frac{d_{0} t}{d \tau}$ is only a function of the scaling variable $\tau=\sigma_{T} . t$. The rationale for this scaling variable is evident from equation (14) by changing variables from $R^{2}$ to $\sigma_{T}$. This scaling is observed at the ISR as can be seen from Figure (6). However, the geometric scaling hypothesis cannot explain the increase in the ratio $\frac{\sigma_{\text {al }}}{\sigma_{\mathrm{T}}}$ with energy as seen by the UA4 experiment at CERN. See Figure (7).

\subsubsection{Eikonal Models}

The fourier transform of the elastic Bcattering amplitude $F(s, t)$ into impact parameter space is written $f(s, b)$, where $8, t$ are the usual Mandelstam vari- 


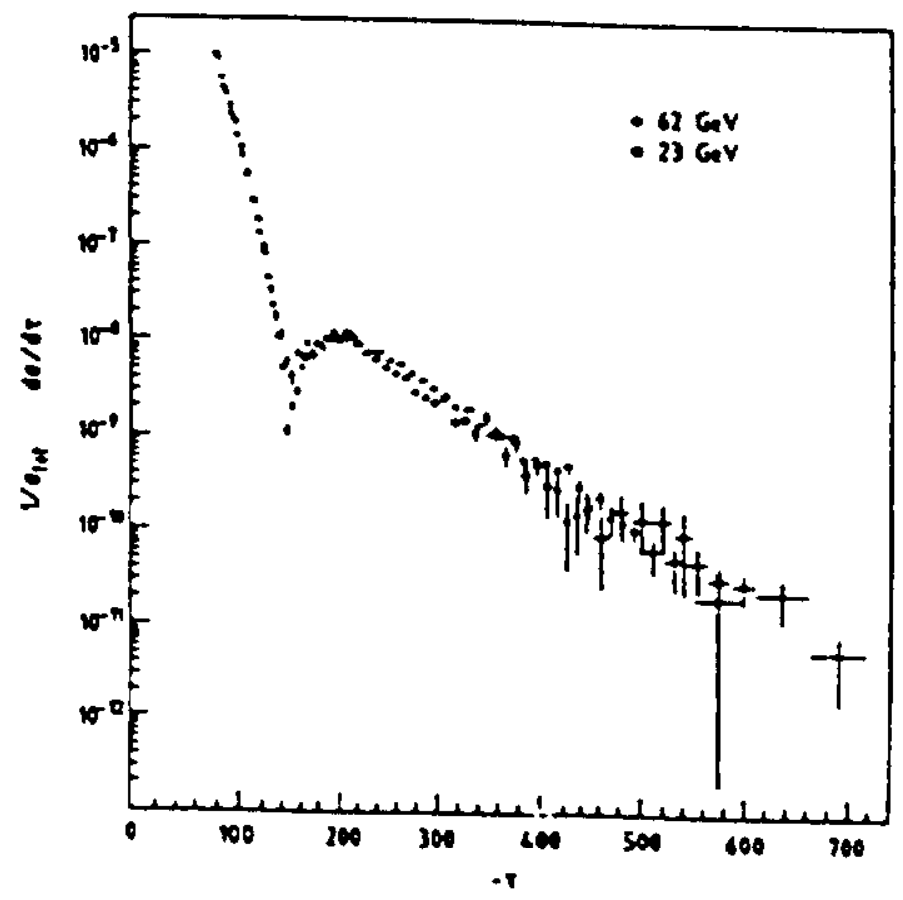

Figure 6: Evidence of geometric scaling at the ISR

ables and $b$ is the impact parameter. $\operatorname{Im}(f(s, b))$ is referred to as the profile function, and represents the hadron opacity as a function of $b$. In the high energy domain, the amplitude tends to be purely imaginary, and unitarity demands $\operatorname{Im}(f) \leq 1 / 2$. This situation is sometimes explicitly expressed in terms of the complex eikonal function $\chi(s, b)[9]$

$$
f(s, b)=\frac{1}{2}\{1-\exp (i \chi(s, b)]
$$

where $\operatorname{Im} \chi \geq 0$. If $R e \chi=0, f$ is purely imaginary and is determined by the opaqueness $\Omega(s, b)=\operatorname{Im} \chi$. In the limit $\Omega \rightarrow \infty$, we obtain the black disc . Eikonal models have been developed further as Factorizing eikonals [10] and seem to have the ability to fit data the best [11] at present. See Figure (8).

At the Tevatron, E710 hopes (Figure 9) to obtain data at the highest energies to discriminate further between the models outlined above. Elastic scattering, being connected via the optical theorem to the total cross section, is a fertile area for new theoretical ideas and will continue to play an important part in our attempts to understand the strong interaction. The 


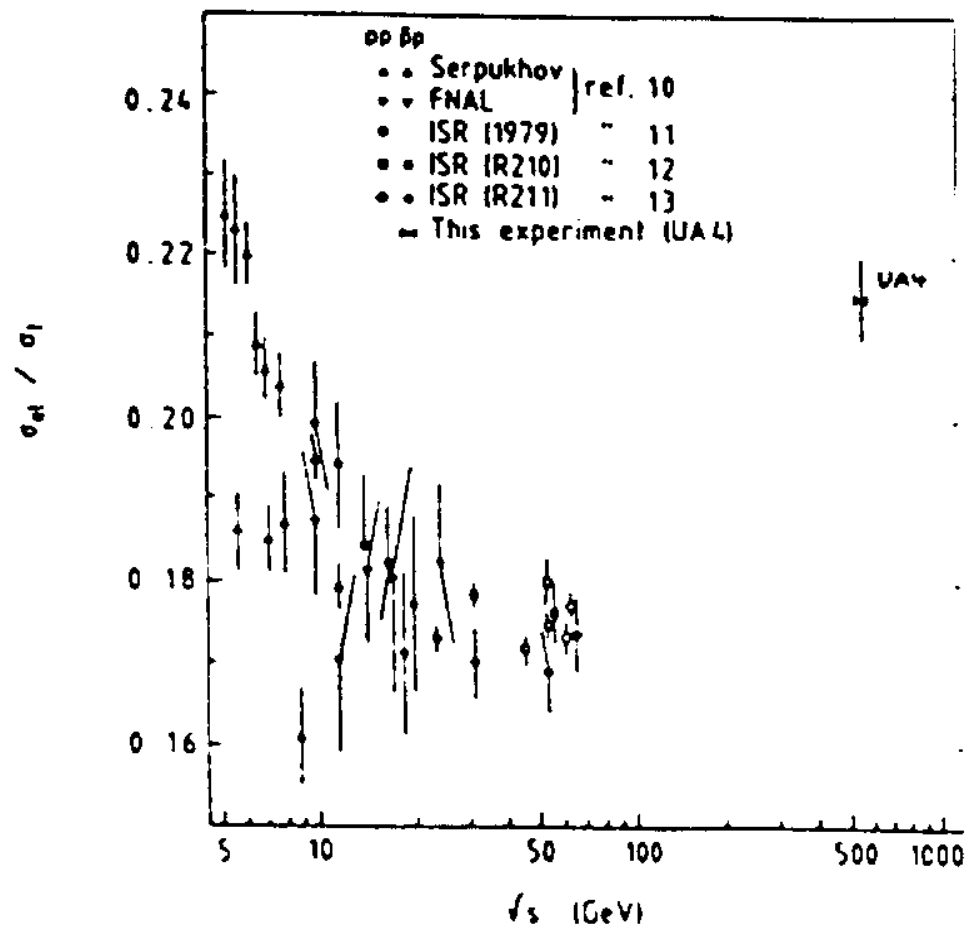

Figure 7: Ratio of elastic to total cross section as a function of $s$ 
models outlined above are essentially non-perturbative in character. QCD so far has yet to make a dent in predicting the basic features of elastic scattering.

\subsection{Inclusive Cross sections}

As one went from CERN PS energies to Fermilab fixed target energies in the early 70's, particle multiplicities became too large in interactions for the processes to be studied exclusively. Instead both experiment and theory concentrated on studying the phenomenology of reactions where one or two particles were measured at any point in phase space and the rest of the reaction products were lumped into an unknown quantity (usually denoted $\mathrm{X}$ ). Such reactions were termed inclusive reactions. Examples are $p p \rightarrow p+X$ and $\bar{p} p \rightarrow \pi+X$. The single particle phase space of a particle of rest mass $m_{0}$ can be written

$$
d L i p s=d^{3} p d E \delta\left(E^{2}-p^{2}-m_{0}^{2}\right)
$$

where $p$ is the momentum and $\mathrm{E}$ the energy of the particle. $\delta$ is the Dirac delta function expressing the condition that the particle is on mass shell. For a fixed momentum $p$, one can integrate out the Dirac delta function by integrating over $\mathrm{E}$.

$$
\int \delta\left(E^{2}-p^{2}-m_{0}^{2}\right) d E=\int \delta(z) \frac{d E}{d z} d z ; z=E^{2}-p^{2}-m_{0}^{2}
$$

This yields $d L i p s=d^{3} p / 2 E$ The Lorentz invariant inclusive cross section is just $d \sigma / d L i p s$ and is written as $\frac{E d^{3} \sigma}{d p^{3}}$. Very often, the invariant inclusive cross section is expressed in terms of variable pairs $\left(y, p_{t}^{2}\right),\left(M^{2}, t\right)$, or $\left(x, P_{t}^{2}\right)$, where $y=0.5 \ln \left(\left(E+p_{l}\right) /\left(E-p_{l}\right)\right)$ is the center of mass rapidity, $p_{l}$ is the center of mass longitudinal momentum and $p_{t}$ is the transverse momentum of the particle under study, $t$ is the momentum transfer squared from the beam (at times the target) to the particle in question and $M^{2}$ is the missing mass squared of the system $\mathrm{X}$ and $x=p_{l} / p_{l} \max \approx 2 p_{l} / \sqrt{(s)}$ is the Feynman $x$ variable of the particle. The third variable $\phi$, which is the azimuthal angle with respect to the beam direction of the particle is usually integrated out since most experiments have no beam (target) polarization. 


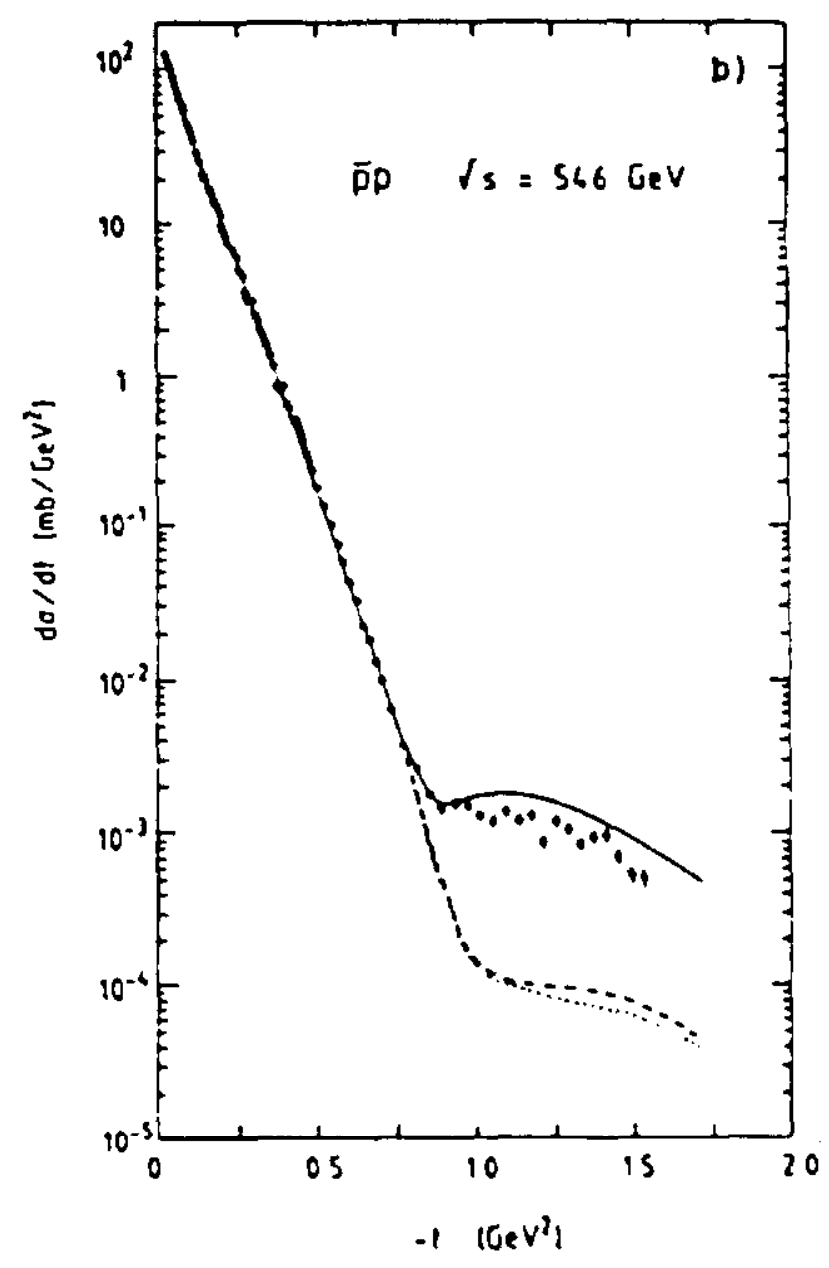

Figure 8: Elastic differential cross section as a function of $-t$ and fits to data 


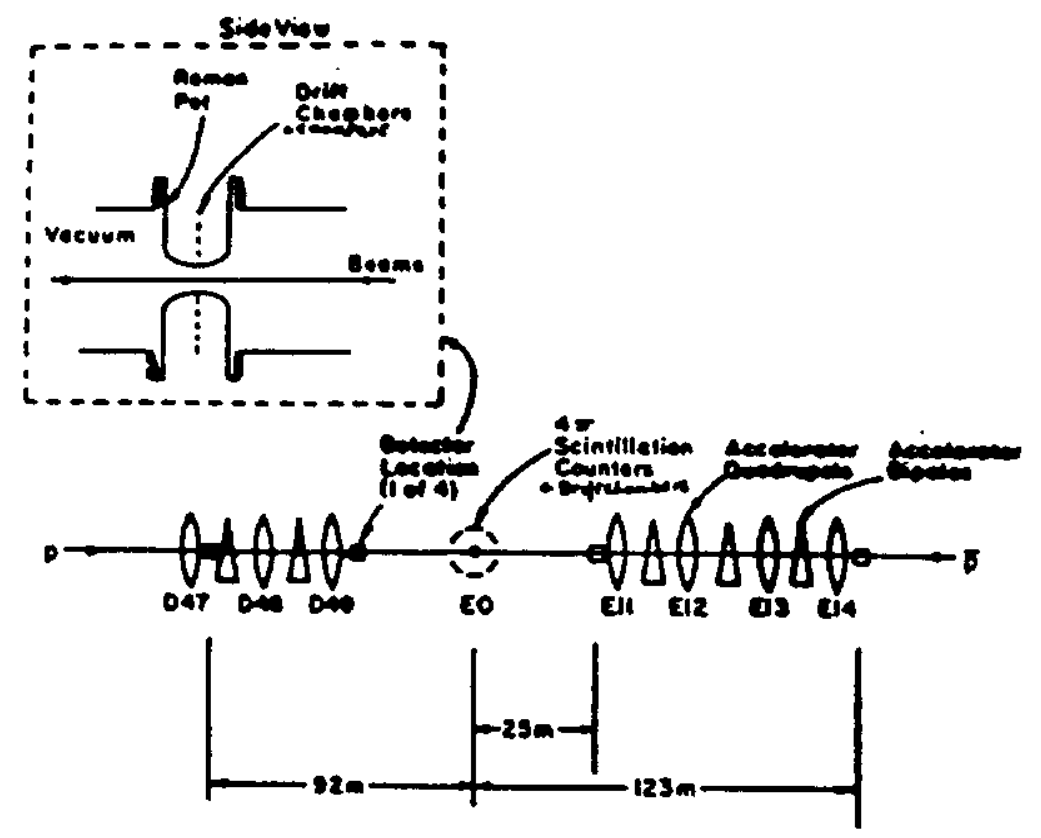

Figure 9: Experimental setup of E710 
The inclusive cross section can be transformed from one set of variables to another by using the Jacobian method. e.g

$$
\frac{E d^{3} \sigma}{d p^{3}}=f(p, \cos \theta)=g\left(x, p_{t}^{2}\right)\left|\frac{\delta\left(x, p_{t}^{2}\right)}{\delta(p, \cos \theta)}\right|
$$

where $\left|\frac{\delta\left(x, p_{t}^{2}\right)}{\delta(p, c o s \theta)}\right|$ is the Jacobian of the transformation. This process leads to the following equivalent expressions for the invariant inclusive cross section.

$$
\frac{E d^{3} \sigma}{d p^{3}} \approx \frac{2 E d^{2} \sigma}{\pi \sqrt{(} s) d x d p_{t}^{2}}=\frac{1 d^{2} \sigma}{\pi d y d p_{t}^{2}} \approx \frac{s d^{2} \sigma}{\pi d M^{2} d t}
$$

At times the pseudo-rapidity $\eta=-\log \tan (\theta / 2)$ is used instead of the rapidity $y$ in experiments which do not measure the energy of the produced particle.

\subsubsection{Diffractive dissociation}

The next simplest process to elastic scattering is diffractive dissociation where either the beam or the target particle remains intact after the collision and the other fragments. The appropriate variables to analyze this process are $M^{2}$ and t, where $M^{2}$ is the mass squared of the fragmenting system and $t$ is the momentum transfer squared of the other particle with respect to its parent. Diffraction dissociation has been treated by perturbative Reggeon calculus [12], QCD approximations [13] , the pomeron-photon analogy of Donnachie and Landshoff[14] and the triple-Regge approach[15].

Figure (10) shows a comparison of UA4 data with ISR data demonstrating the existing of scaling in the invariant cross section $\frac{1}{\pi} d^{2} \sigma / d t d\left(M^{2} / s\right)$ plotted as a function of $M^{2} / \mathrm{s}$. Scaling seems to be good to within 10$20 \%$ at two fixed values of $|t|$. Figure (11) shows the $1 / M^{2}$ falloff in the diffractive peak as would be expected in the triple-Regge picture. It is seen that the $1 / M^{2}$ behavior persists between the ISR and the $s \bar{p} p s$. One of the outstanding questions about diffractive fragmentation is whether the fragmenting system emits particles isotropically, or whether the system decays according to longitudinal phase space. The pseudo rapidity spectra obtained by UA4 show conclusively that longitudinal phase space prevails over isotropic decay[1]. This is predicted by multiperipheral models[16] and seems to rule out fireball models that predict isotropic decay[17]. 


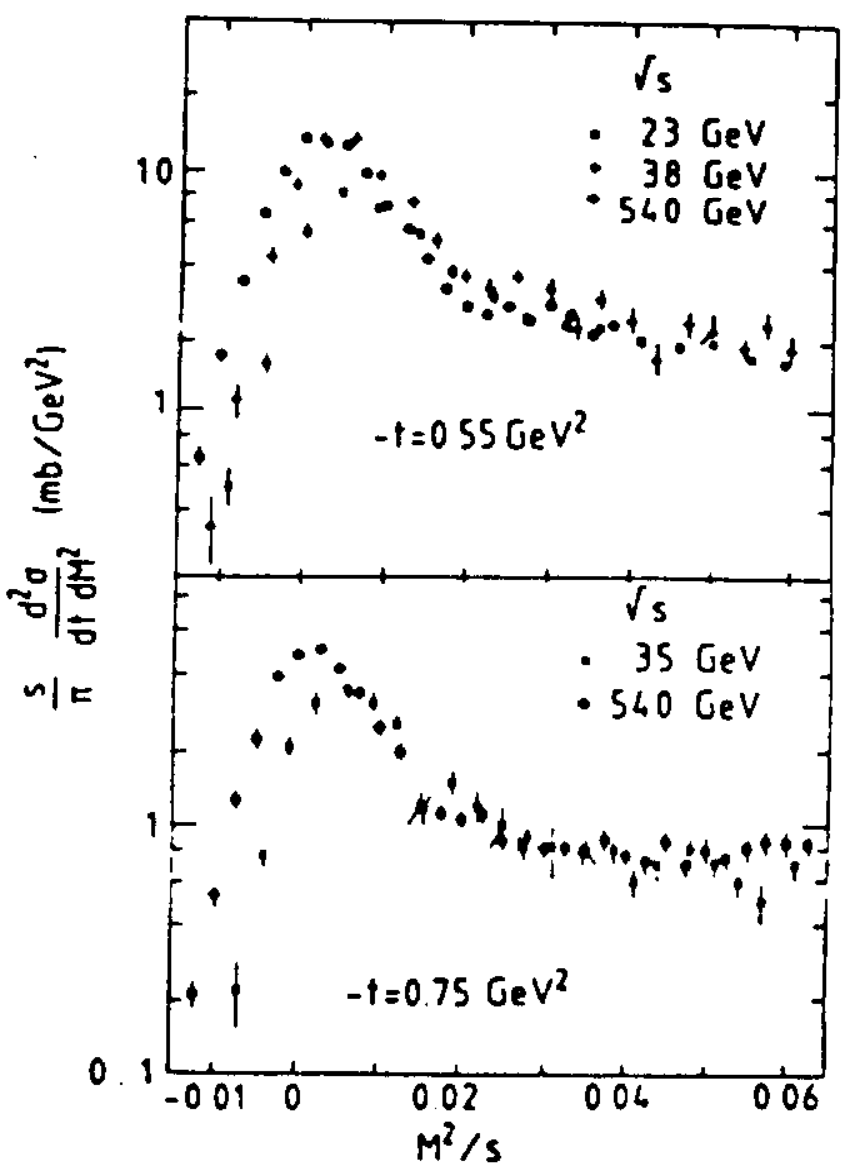

Figure 10: Scaling of the invariant diffractive cross section at two values of $t$ between ISR and SPS energies 


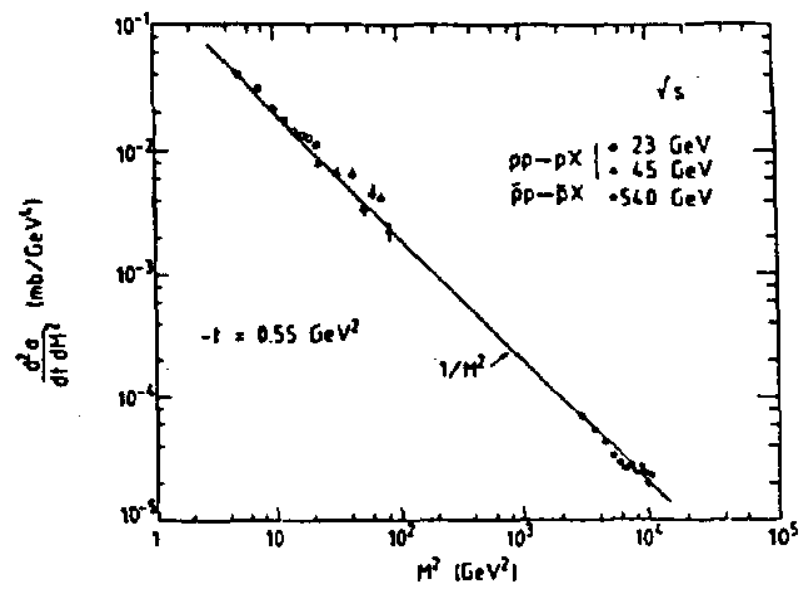

Figure 11: $1 / M^{2}$ scaling behavior in the energy range ISR to SPS

\subsubsection{Central production}

The most copiously produced particles in high energy collisions are pions. The inclusive cross section $E d^{3} \sigma / d p^{3}$ can be analyzed in terms of the variables $\left(y, p_{t}^{2}\right)$. The most striking feature of these collisions is that the $p_{t}$ of the pions produced is limited to $\approx 400 \mathrm{MeV}$, even though much larger values are allowed by phase space considerations. This gives rise to the notion of longitudinal phase space, where one imagines that there is a tube in $p_{t}$ space that has radius $400 \mathrm{MeV}$ which extends in rapidity space. As the energy increases, the length of the tube in rapidity space grows logarithmically as $\ln \left(s / m_{\pi}^{2}\right)$. The inclusive cross section, one assumes, will scale with energy giving rise to the "rapidity plateau". This implies that if $\sigma_{\text {inel }}$ is constant with energy, the mean multiplicity of pions will grow logarithmically with energy, since

$$
\sigma_{\text {inclusive }}=<n_{*}>\sigma_{\text {inel }}
$$

However, we know from experiment that $\sigma_{\text {ined }}$ grows as $\ln ^{2}(s)$, and that $\left\langle n_{\pi}\right\rangle$ grows as $\ln (s)$. This must imply that the invariant inclusive cross 


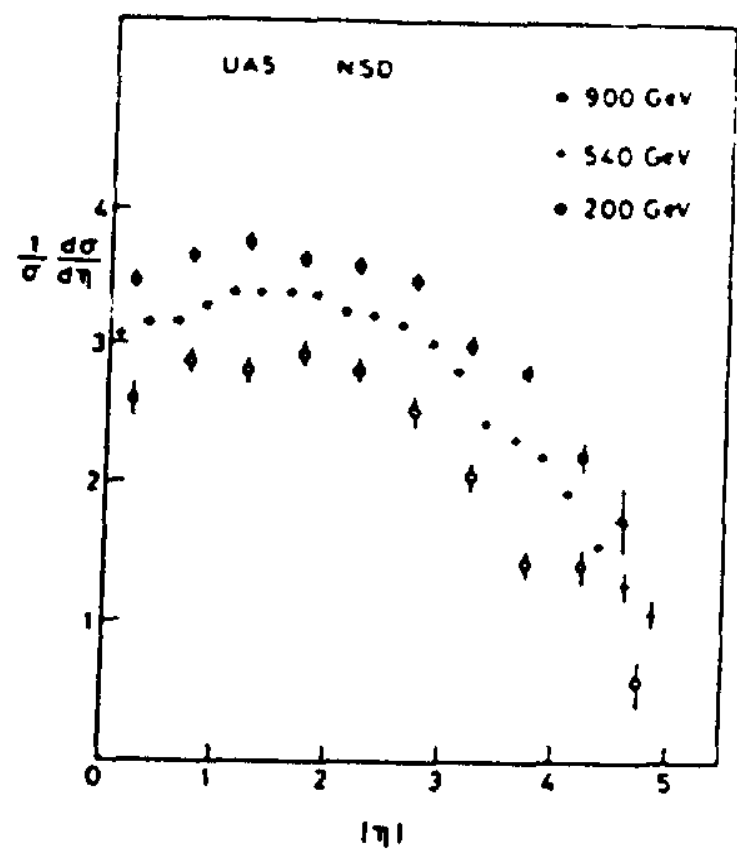

Figure 12: Pseudo-rapidity distributions for non-diffractive events at 200 , 540 and $900 \mathrm{GeV}$

section cannot scale with 8 for a constant $y$. i.e. the rapidity plateau must grow with energy. This is indeed found to be 8o. The UA5 experiment[18] has reported pseudo-rapidity distributions as a function of $\sqrt{(s)}$. Pseudorapidity is used since theirs is a non-magnetic detector. Figure(12) shows a clear rise in the rapidity plateau as a function of center of mass energy in the range $200 \mathrm{GeV}$ to $900 \mathrm{GeV}$. The rise in the center point of the pseudorapidity distribution $\left(\frac{1 d \sigma}{\sigma d n}\right)_{0}$ is plotted as a function of $\sqrt{(s)}$ in Figure (13). It is clear that scaling in pseudo-rapidity, which is equivalent to Feynman $x$ scaling, breaks down. Both the diffractive dissociation and the central production cross sections will be extended to Fermilab energies by the CDF detector shortly.

\subsection{Multiplicity distributions}

Multiplicity distributions are perhaps the simplest things that can be measured and studied in hadronic interactions. They have long been the study of people looking for scaling laws and other regularities. One of the first 


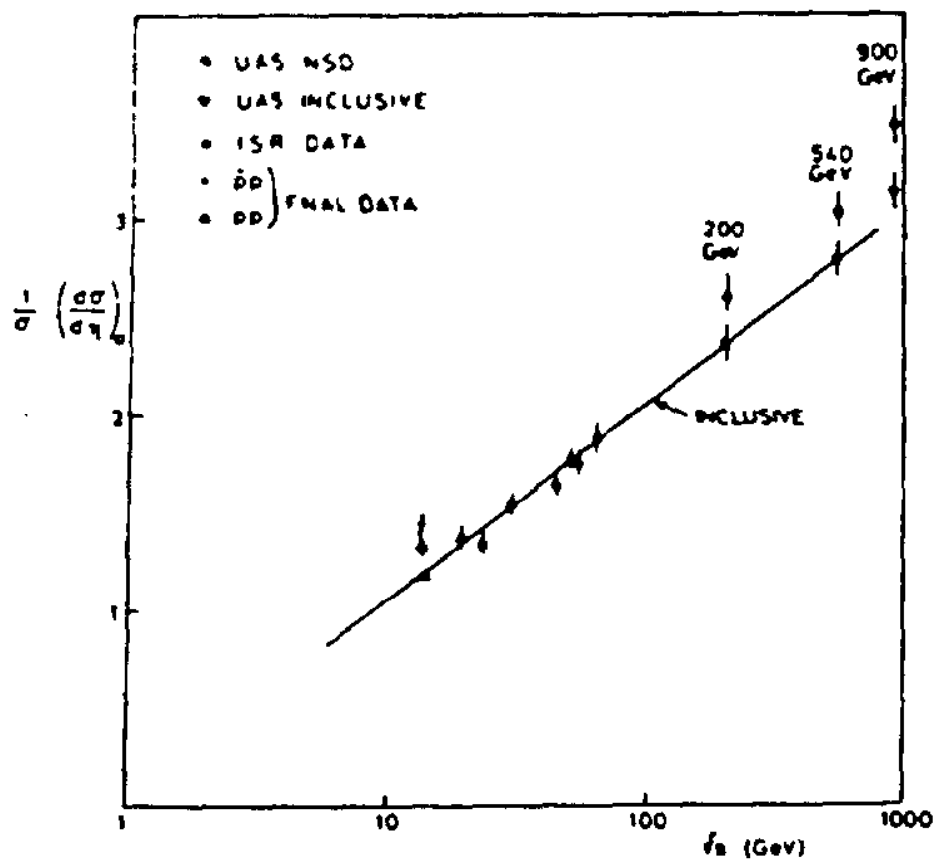

Figure 13: Dependence of the Central rapidity density for inclusive data from Fermilab energies up to $900 \mathrm{GeV}$

regularities to be discovered was the Wroblewski regularity[19] which stated that for $p p$ collisions

$$
D=0.576(<n>-1)
$$

where $\left.D\left(D=\sqrt{(}<n^{2}>-<n>^{2}\right)\right)$ is the dispersion of the inelastic multiplicity distributions, and $\langle n\rangle$ is the average multiplicity. Also during this time, another regularity was derived[20] assuming Feynman scaling for all semi-inclusive cross sections. Following the initials of the authors, this scaling, commonly called KNO scaling, states

$$
\frac{\sigma_{n}(s)}{\sigma_{\text {inal }}(s)} \rightarrow \frac{1}{<n\rangle} \psi\left(\frac{n}{<n\rangle}\right)
$$

the limit being reached for infinite energy. It was shown by Slattery[21] that KNO scaling was valid for $p p$ interactions in the energy range $50 \mathrm{GeV}$ $303 \mathrm{GeV}$ incoming beam momentum. See Figure (14). Subsequently, this was extended to other particle types[22] and up to ISR energies[23]. It was therefore somewhat of a surprise when the UA5 collaboration [24] showed clear evidence of KNO scaling violations (Figure(15) at spps energies. UA5 


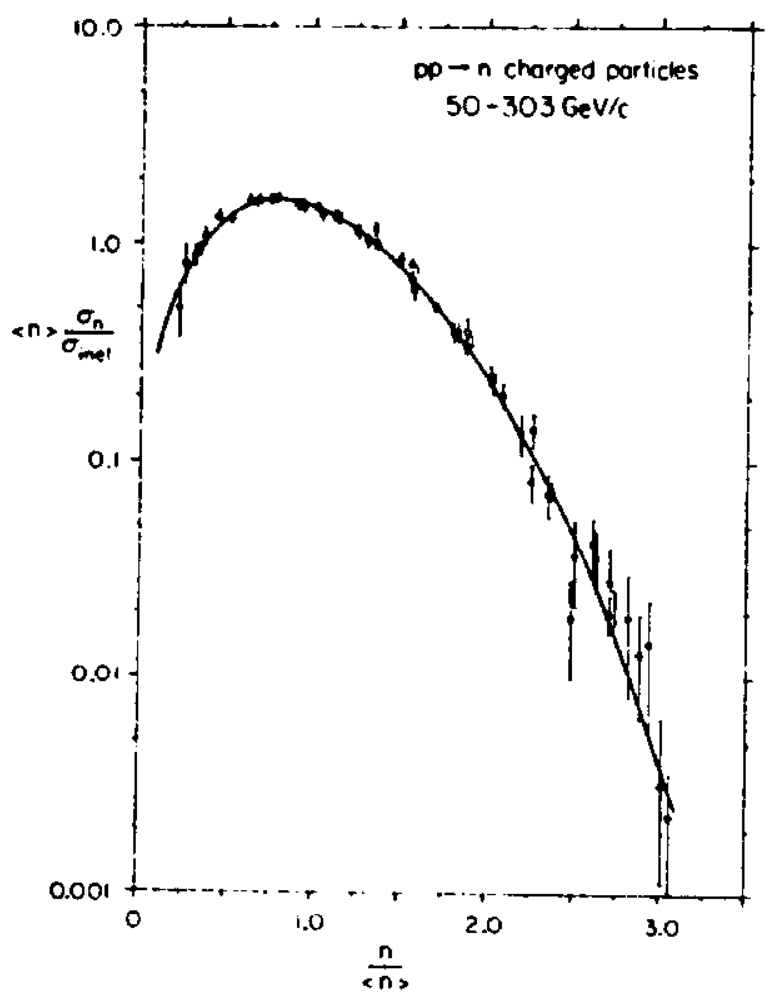

Figure 14: KNO scaling at Fermilab fixed target energies

subsequently[25] showed that all charged particle multiplicity distributions for the non-single diffractive component of pp reactions at $540 \mathrm{GeV}$ and $p p$ reactions above $\approx 10 \mathrm{GeV}$ are remarkably well described by the negative binomial distribution.

$$
P_{n}=C_{k}^{n+k-1}\left(\frac{\bar{n} / k}{1+\bar{n} / k}\right)^{n} \frac{1}{(1+\bar{n} / k)^{k}}
$$

where the two parameters $(\pi, k)$ are functions of energy.

Figure (16) shows an example of the goodness of fit of this distribution to data at the ISR energies. Figure(17) shows the energy dependence of the parameter $k$. It can be seen that $\mathbf{k}$ decreases with energy and does not seem to reach any asymptotic value.

The first two moments of the negative binomial distribution can be written $\left(c_{q}=<n^{2}>/<n>9\right)$

$$
c_{2}=1+\left(\frac{1}{\bar{n}}+\frac{1}{k}\right) ; c_{3}=1+3\left(\frac{1}{\bar{n}}+\frac{1}{k}\right)+\left(\frac{1}{\bar{n}}+\frac{1}{k}\right)^{2}+\frac{1}{k}\left(\frac{1}{\bar{n}}+\frac{1}{k}\right)
$$




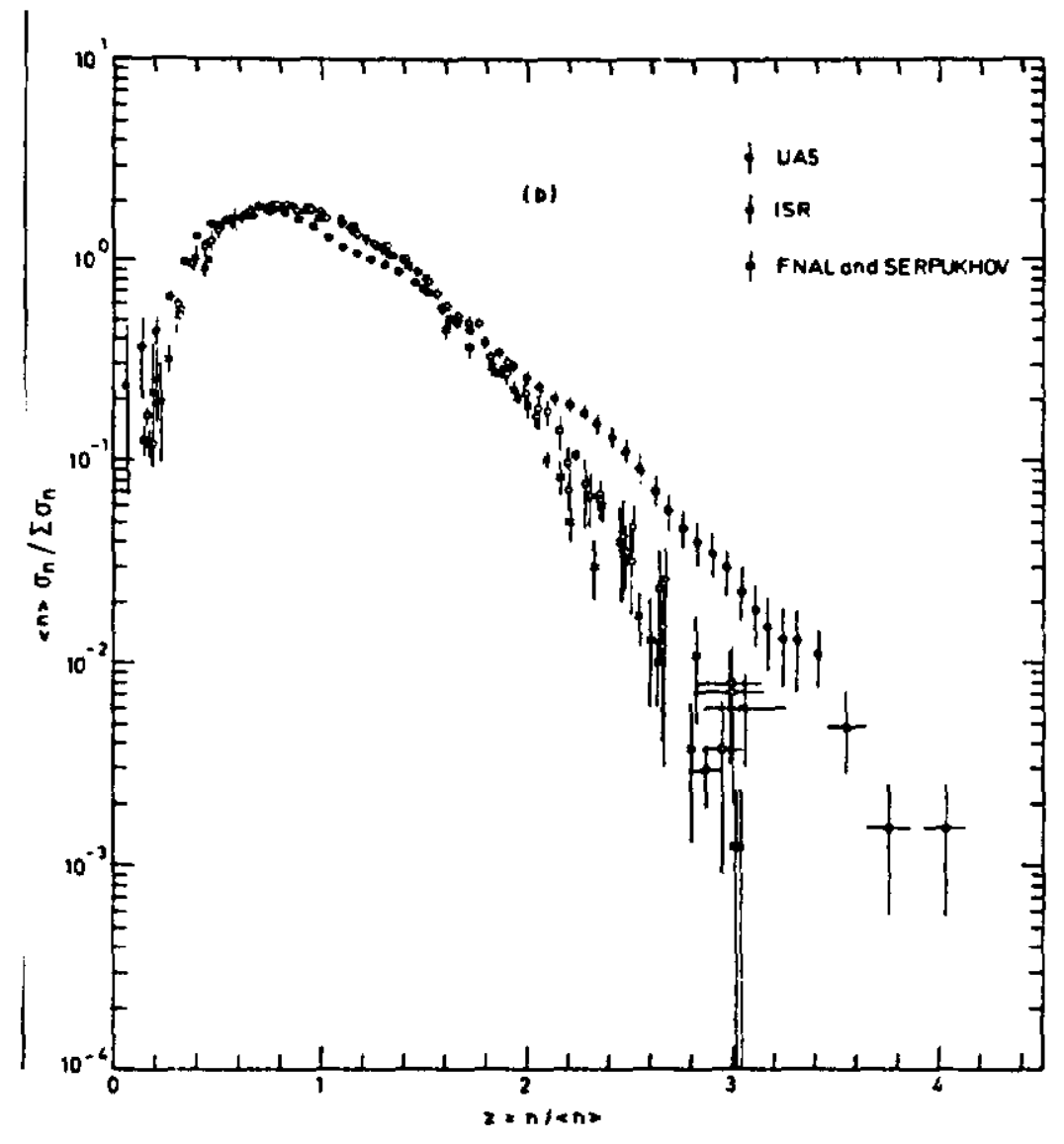

Figure 15: KNO scaling violation observed by UA5 


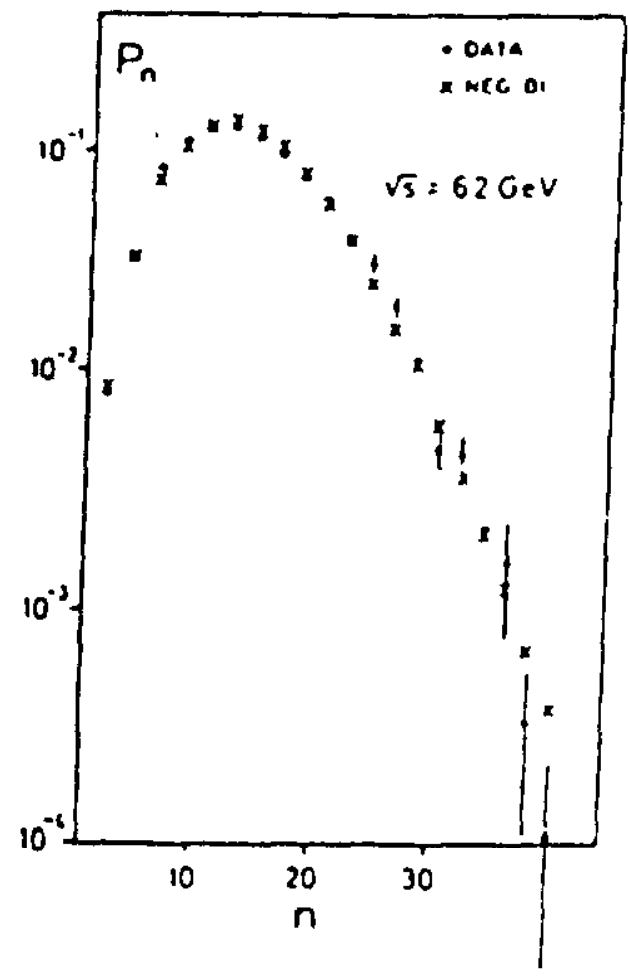

Figure 16: Fit of negative binomial distribution to ISR data at $62 \mathrm{GeV}$

For KNO scaling to hold all $c_{n}$ should be constant with energy. Figure(18) shows a plot of $c_{2}-1$ versus $\sqrt{(s)}$. Approximate KNO scaling results when $\left(\frac{1}{\hbar}+\frac{1}{k}\right)$ is near the broad minimum in the energy range $10<$ $\sqrt{(8)}<62 \mathrm{GeV}$. Figure (19) shows the predicted probabilities assuming negative binomial distribution for SSC energies at $\sqrt{(s)}=40 \mathrm{TeV}$. The probability of getting high multiplicity events is much larger in the negative binomial scenario than if KNO scaling were to hold.

There have been many attempts to explain KNO scaling violations. The QCD based approsch splits the cross section into two terms. One due to a non-jet background and the other due to QCD mini-jets.[26]. The negative binomial only arises approximately in these models. There are other models where it arises naturally[27]. The parameter $k$ finds a physical interpretation in these models as the number of independent emitters. These should intuitively increase with energy. Data shows, however, that $k$ decreases with energy.

The CDF detector at Fermilab is currently taking minimum bias data and should be able to extend these ideas to the $2 \mathrm{TeV}$ regime. 


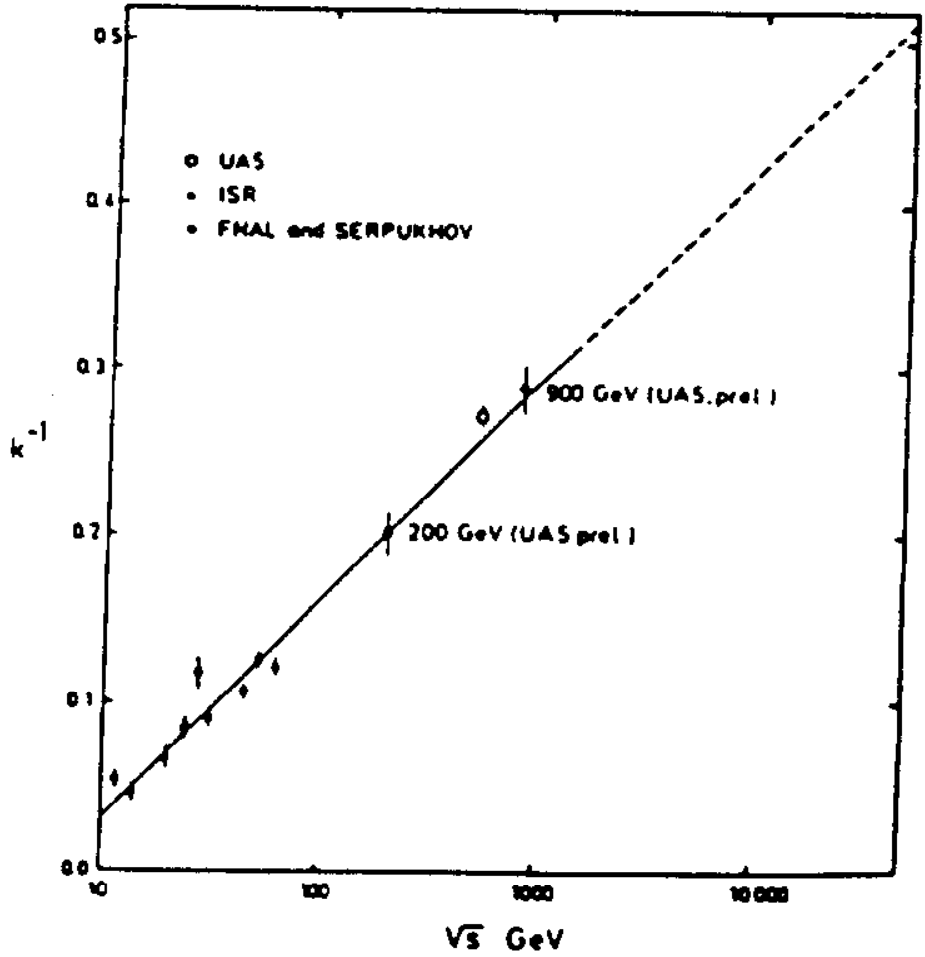

Figure 17: Energy dependence of the parameter $k$ 


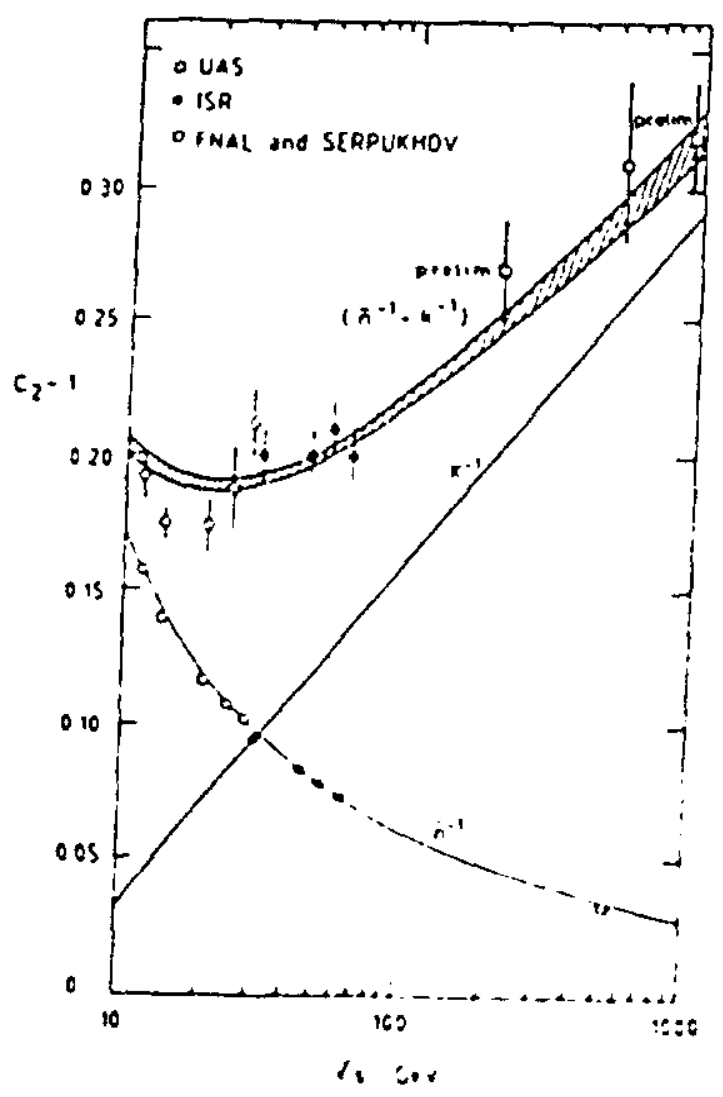

Figure 18: $c_{2}-1$ versus sqrt(s) 


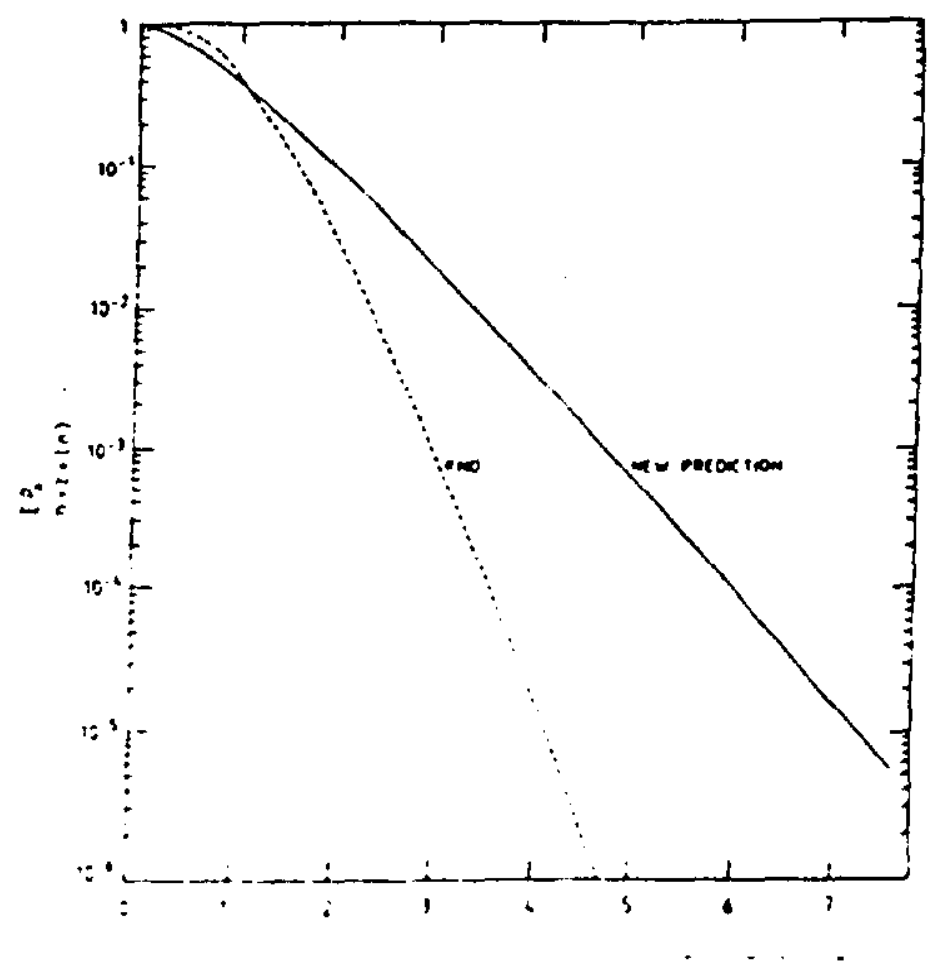

Figure 19: Multiplicity predictions for SSC energies

\subsection{Quark Gluon Plasma}

In lattice $Q C D,[28]$, it can be shown that there exists a phase transition at "high temperature" (energy) in the quark gluon system which liberates quarks in a nucleus from being confined to individual nucleons and permits them to traverse the whole of the nucleus freely. Such a state of matter has been termed quark-gluon plasma. E735 is currently setting up in the C0 area (see Figure 20) to look for quark gluon plasma effects at Fermilab. They plan to do this by measuring $\left\langle p_{t}\right\rangle$ of the particles emitted as a function of $d n / d y$. At present, $<p_{t}>$ rises with $d n / d y$ and plateaus. If it rises again after the plateau, then this may be interpreted broadly as evidence for the quark gluon plasma phase transition. Results are eagerly awaited.

To conclude, minimum bias physics is interesting and not terribly well understood. Most regularities observed so far seem to be violated at higher energies. Considering the fact that most of the strong interaction cross section lies in these events, our present state of understanding of the strong force cannot be said to be anything other than rudimentary. 


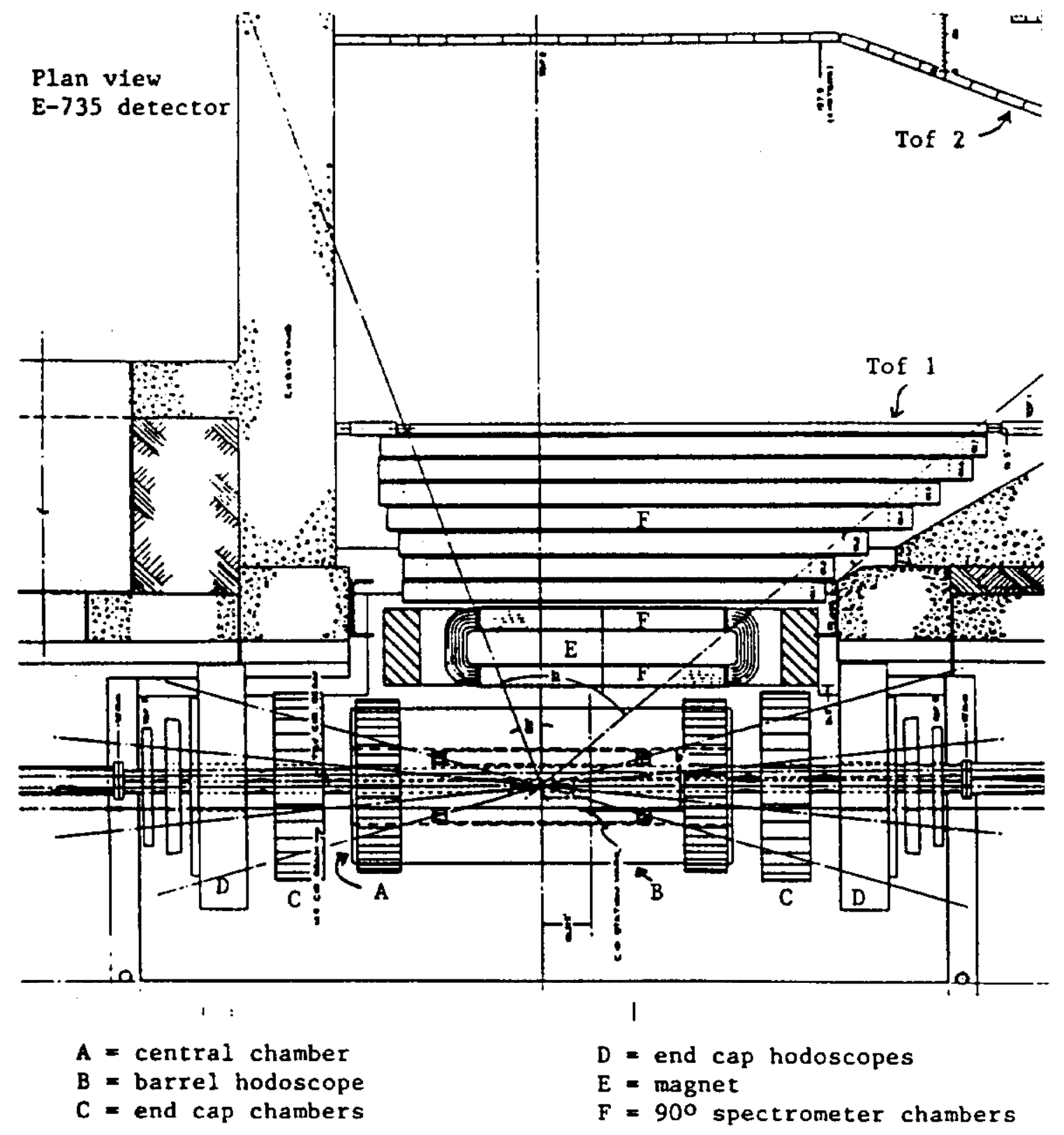

Figure 20: The E735 detector at Fermilab 


\section{QCD Jet Physics}

With the success of gauge theories to describe electrodynamics and the weak interaction, it was only natural that a search should be made for gauge theory candidates to describe the strong interaction. The spin-statistics problem in the quark model for baryons as well as the magnitude of the cross section for $e^{+} e^{-}$annihilations into hadrons pointed to the existence of the color degree of freedom for quarks [29]. It then became natural to attempt to build a gauge theory of strong interactions based on the color gauge group $S U(3)_{c}[30]$. Since the strong coupling constant is large, perturbative methods do not converge in general. Further progress came with the realization that the strong coupling constant varied with the energy scale with which the hadron was probed and that the coupling became weaker, the shorter the distance scales that were probed. This central result [31] is known as asymptotic freedom, and is directly responsible for making collider experiments the natural testing ground for perturbative QCD predictions.

Independently of the gauge theory approach, analysis of deeply inelastic lepton hadron scattering experiments led to ideas that hadrons were composed of quasi-free point like particles (termed partons by Feynman). This approach led to predictions of Bjorken scaling [32] which were demonstrated experimentally to be true in ep scattering experiments at SLAC [33]. Interpretations of scale invariance in terms of the parton model was first given by Feynman [34]. Analysis of production of oppositely charged lepton pairs in hadron collisions led Drell and Yan [35] to suggest that such pairs were the result of quark -antiquark fusion into a virtual photon that subsequently decayed into the lepton pair. These developments in turn led to a general description of hadronic high $p_{t}$ processes in terms of parton constituents by Field and Feynman [36]. An excellent review of early QCD is by Quigg [37].

The partons, though quasi-free at high energy (short times and distances) are nevertheless forever confined within the nucleon. The only allowed states are color singlets. Color is mediated by the carriers of the color force, the vector gluons of which there are eight in number. One of the predictions of the quark parton model is that, although hadron-hadron 
collisons result in low $p_{t}$ minimum bias physics most of the time, once in a while partons from colliding hadrons will participate in a hard collision. The force of the collision will be sufficient to cause the partons to fly apart at high transverse momentum. By the time they separate in distance by roughly $1 \mathrm{fermi}$, the forces causing color conservation take over, causing a chain of hadronic fragmentation (see figure (21). However, the particles come grouped in clusters along the initial directions of the final state partons i.e. back to back in the transverse plane. This type of topology is termed "jets" in the jargon.

In this chapter we will discuss in order

- the quark parton model equations for jet production

- Structure functions and scale breaking

- Observation of jets. 2 Jets, 3 Jet and multi jet final states. Inital and final state bremsstrahlung, fragmentation

- QCD jets as a source of background at hadron colliders

\subsection{Quark parton model}

\subsubsection{Basic assumption}

The basic assumption of the quark parton model is that the inclusive cross section $a b \rightarrow c+X$ where $\mathrm{c}$ is a final state with sufficiently large $Q^{2}$ (e.g. 2 Jets, $W / Z$ production) is given by (see Figure (22))

$$
d \sigma(a+b \rightarrow c+X)=\sum_{i, j} f_{i}^{(a)} f_{j}^{(b)} \hat{\sigma}\left(i j \rightarrow c+X^{\prime}\right)
$$

where $f_{i}^{(a)}$ is the probability of finding the parton $i$ in the hadron $a$ and $\hat{\sigma}$ is the elementary parton-parton cross section to form $c$. The essence of the parton model is to treat hadrons as a collection of essentially free partons, and a hadron collider essentially becomes a factory for producing parton-parton collisions. The equation (25) should be modified to take into 


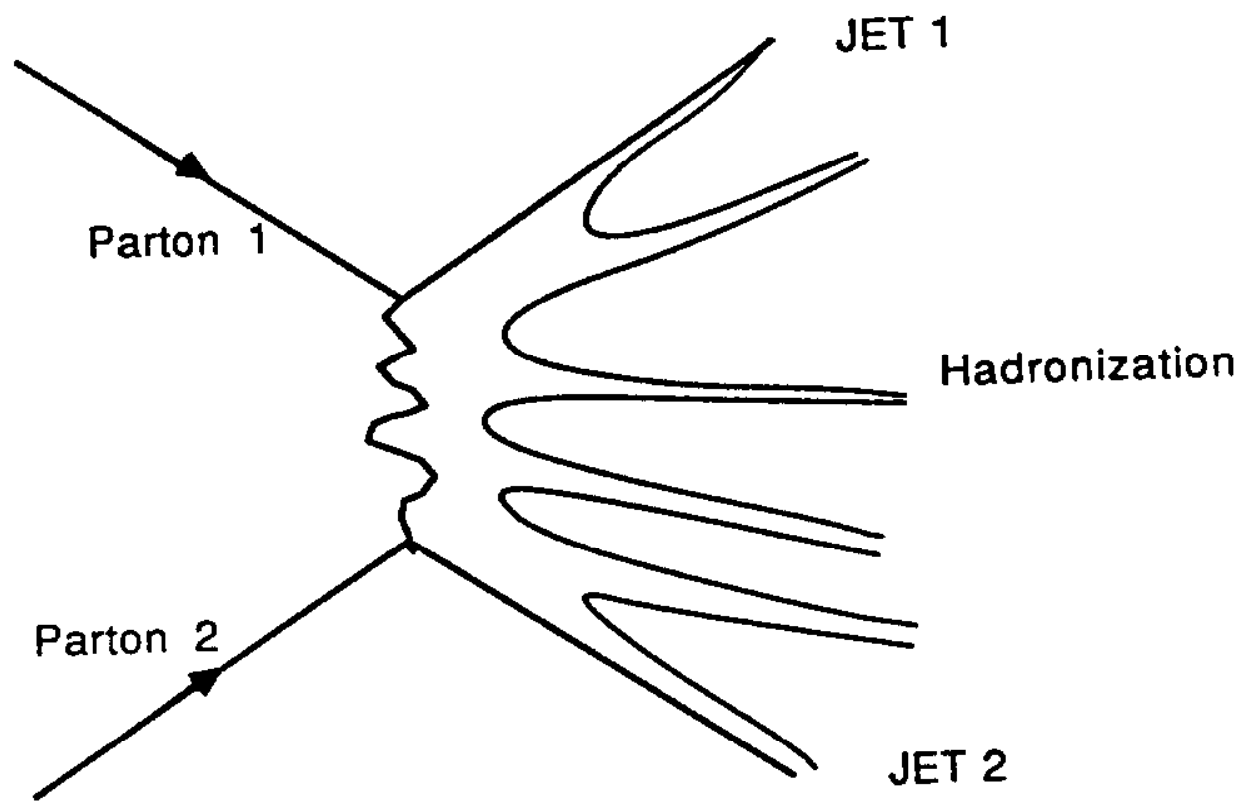

Figure 21: Jet fragmentation into hadrons

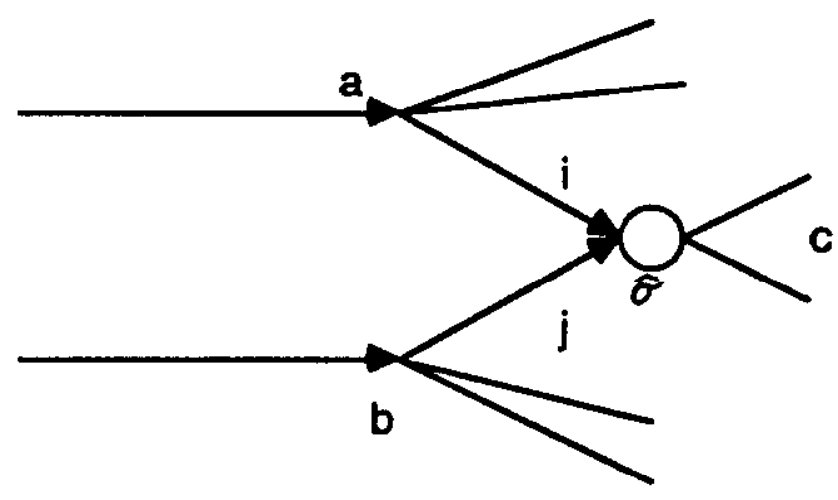

Figure 22: Parton model process 


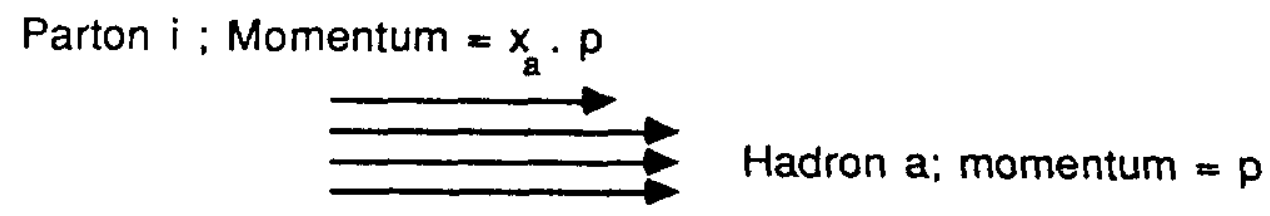

Figure 23: Momentum fraction $x$ carried by parton $i$

consideration the possibility that parton $i$ may come from particle $b$. The equation then becomes

$$
d \sigma(a+b \rightarrow c+X)=\sum_{i, j} \frac{1}{1+\delta_{i j}}\left[f_{i}^{(a)} f_{j}^{(b)}+i \longleftrightarrow j\right\} \hat{\sigma}\left(i j \rightarrow c+X^{\prime}\right)
$$

where the Kronecker delta function $\delta_{i j}$ is introduced to avoid double counting when the parton $i$ is the same as the parton $j$. Parton $i$ carries momentum fraction $x_{a} P$ of the hadron with momentum $P$. See Figure (23). Tranoverse momentum of the parton within the hadron is ignored at this stage. It is clear that $0 \leq x_{a} \leq 1$ and that $\sum x_{a}=1$ by momentum conservation. One can readily show that the energy of the parton (with momentum fraction $x_{a}$ ) in the rest frame of the hadron is $m_{0} x_{a}$ where $m_{0}$ is the rest mass of the hadron. So $x_{a}$ may be thought of as the fraction of the mass of the hadron carried by the parton. The probabilities $f_{i}^{(a)}$ may be written as the distributions $f_{i}^{a}\left(x_{a}\right) d x_{a}$ and the equation (26) then can 
be re-written

$\frac{d^{2} \sigma}{d x_{a} d x_{b}}(a+b \rightarrow c+X)=\sum_{i, j} \frac{1}{1+\delta_{i j}}\left[f_{i}^{(a)}\left(x_{a}\right) f_{j}^{(b)}\left(x_{b}\right)+i \longleftrightarrow j\right] \hat{\sigma}\left(i j \rightarrow c+X^{\prime}\right)$

The distributions $f\left(x_{a}\right)$ are commonly known as "structure functions".

In the center mass of the $a b$ system, (see figure (24)), $\hat{s}$ is the $i j$ center of mass energy and $s$ is the $a b$ center of mass energy. Neglecting all masses,

$$
p^{*}=\sqrt{(s) / 2}
$$

Define the Feynman $\mathbf{x}$ of the $i j$ system as

$$
x=\frac{2 p_{\text {long }}(i, j)}{\sqrt{(s)}}=\frac{2\left(x_{a}-x_{b}\right) p^{*}}{\sqrt{(s)}}=x_{a}-x_{b}
$$

also,

$$
\hat{s}=E_{i j}^{2}-P_{i j}^{2}=p^{* 2}\left[\left(x_{a}+x_{b}\right)^{2}-\left(x_{a}-x_{b}\right)^{2}\right]=s x_{a} x_{b}
$$

leading to

$$
x_{a} x_{b}=\frac{\hat{s}}{s} \equiv \tau
$$

So given $x$ and $\tau$, which are measurable in the final state, the momentum fractions $x_{a}$ and $x_{b}$ can be deduced.

$$
\begin{aligned}
& x_{a}=\frac{1}{2}\left[\sqrt{\left(x^{2}+4 \tau\right)}+x\right] \\
& x_{b}=\frac{1}{2}\left[\sqrt{\left(x^{2}+4 \tau\right)}-x\right]
\end{aligned}
$$

One may note the following general features of this model. If $M$ is the mass of the final state system $c$ (i.e $M^{2}=\hat{s}$ ), $\tau=M^{2} / s$; so as $s \rightarrow \infty, \tau \rightarrow 0$. Since $x_{a} x_{b}=\tau$, and since since both $x_{i, j}$ are positive, on average both $x_{a}$ and $x_{b}$ will tend towards zero for large $s$. So as $s$ rises, partons $w_{\text {tith smaller }}$ momentum fraction of the hadron (so called "wee" partons) will contribute to processes for a given $M^{2}$ scale. By demanding the Feynman $x$ of the system $c$ to be fixed, one can determine the momentum fractions $x_{i, j}$ of the contributing partons for any give $s$. Since the maximum value of $x_{i, j}$ is unity, the minimum value must be $\tau$. So for a given value of $\tau, x_{a}, x_{b}$ vary between $\tau$ and 1 . 


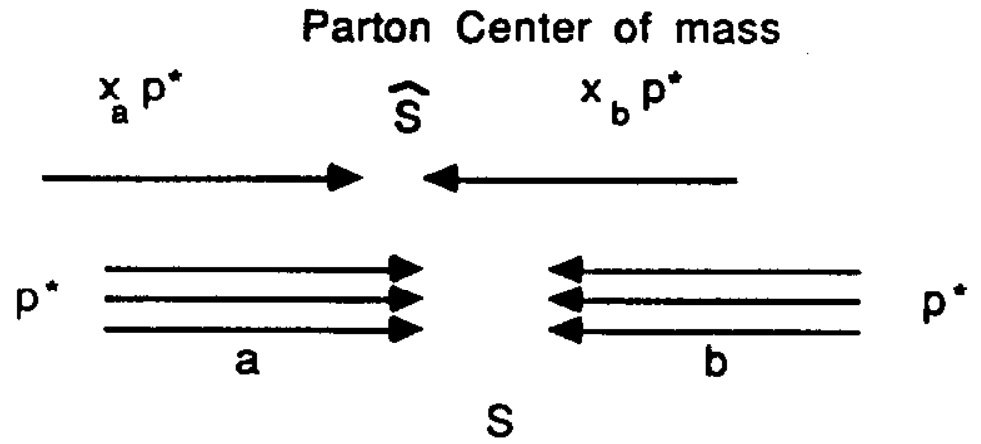

Hadron Center of mass

Figure 24: Center of mass of the ab system

\subsubsection{Valence and sea quark distributions}

The proton is made up of 2 up quarks and 1 down quark. These are the "valence" quarks that make up the proton. Defining $u(x) d x$ to be the probability of finding an up quark of momentum fraction between $x$ and $x+d x$ and $d(x) d x$ to be the corresponding function for the down quarks, the normalization conditions would read

$$
\int_{0}^{1} u(x) d x=2 ; \int_{0}^{1} d(x) d x=1
$$

Also, from momentum conservation, we would write

$$
\int_{0}^{1} x(u(x)+d(x)) d x=1
$$

The above equation would be true if the only partons in the system were valence quarks. From the existence of Drell-Yan processes in pp interactions, one can deduce the existence of an anti-quark sea in the proton. This leads to anti-quark structure functions for $\bar{d}(x), \bar{u}(x), \bar{s}(x)$ for the down, up and strange antiquark sea distributions of the proton. At high $Q^{2}$, the 
charm, bottom and top seas can become significant. The sum rules now become,

$$
\int_{0}^{1}[u(x)-\bar{u}(x)] d x=2 ; \int_{0}^{1}[d(x)-\bar{d}(x)] d x=1 ; \int_{0}^{1}[s(x)-\bar{s}(x)] d x=0
$$

One can measure these structure functions from deeply inelastic scattering reactions of the type $(\nu p \rightarrow \mu+X ; \mu p \rightarrow \mu+X)$ etc. When the momentum sum rule is evaluated for these structure functions, one finds

$$
\int_{0}^{1} x[u(x)+d(x)+s(x)+\bar{u}(x)+\bar{d}(x)+\bar{s}(x)] \approx 0.5
$$

At this point, one is forced to introduce the gluon structure function $G(x)$, in order to conserve momentum, such that

$$
\int_{0}^{1} x G(x) \approx 0.5
$$

Since we use either virtual W's or virtual $\gamma$ 's in deeply inelastic scattering to probe the proton, we cannot measure the gluon structure function directly by these methods. The evolution of the gluon structure function is predicted by the Altarelli-Parisi equations which will be dealt with later in this chapter, under the subsection entitled 'scale breaking'.

\subsubsection{2 jet production}

The primary process for two jet production is the hard scattering of partons $i$ and $j$ with center of mass energy $\hat{s}$ to produce final state partons 2 and 4 (Figure (25) that fragment into final state jets. The Mandelstam variables

$$
\begin{gathered}
\hat{s}=\left(p_{1}+p_{3}\right)^{2} \\
\hat{t}=\left(p_{1}-p_{2}\right)^{2}=-\frac{3}{2}(1-\cos \theta) \\
\hat{u}=\left(p_{1}-p_{4}\right)^{2}=-\frac{3}{2}(1+\cos \theta)
\end{gathered}
$$

(where the squaring indicates 4 vector dot products) define the kinematics of the scattering. Then the two jet differential cross section can be written

$$
\frac{d^{3} \sigma}{d x_{a} d x_{b} d \hat{t}}=\sum_{i j} \frac{1}{1+\delta_{i j}}\left[f_{i}^{a}\left(x_{a}\right) f_{j}^{b}\left(x_{b}\right)+i \leftrightarrow j\right\} \frac{d \sigma_{i j}}{d \hat{t}}
$$




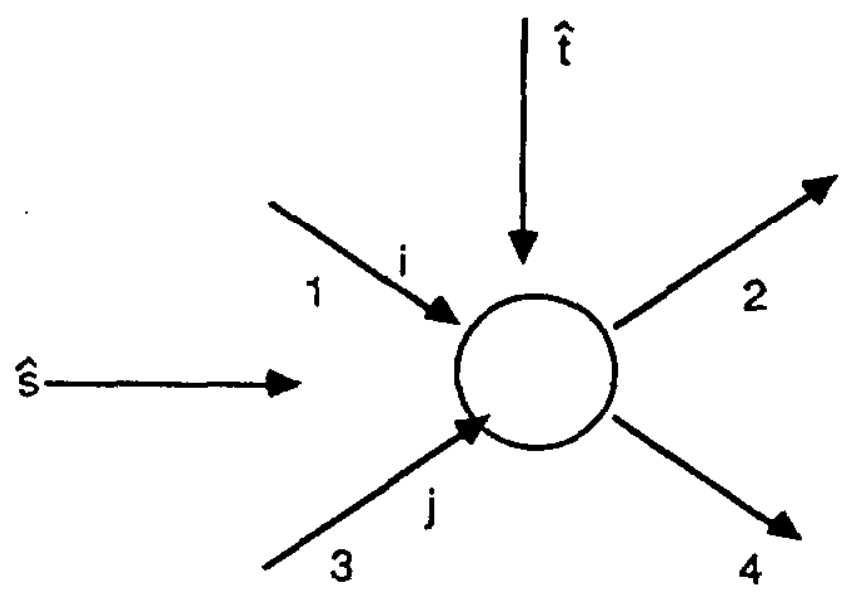

Figure 25: Mandelstam Variables of 2 Jet production

The above equation is expressed in terms of the variable set $\left(x_{a}, x_{b}, \hat{t}\right)$. It can be transformed into any other variable set using the appropriate Jacobian. A commonly used variable set is $y_{1}, y_{2}, p_{t}$ where $y_{1}, y_{2}$ are the rapidities of the two jets and $p_{t}$ is the transverse momentum of one of the jets. The Born diagrams giving rise to the 2 Jet cross section are shown in Figure (26). The numbers at the vertices give the relative couplings ([2]). In general

$$
\frac{d \sigma}{d \hat{t}}=\frac{\pi \alpha^{2}}{\hat{s}^{2}}|\Lambda|^{2}
$$

For $\hat{t}$ channel exchange, $|A|^{2} \approx 1 / \hat{t}^{2}$ and for $\hat{s}$ channel exchange $|A|^{2} \approx$ $1 /(\vec{s})^{2}$. Since for collider experiments, $\hat{t}<\hat{s}$, it is easy to see that $\hat{t}$ channel exchanges dominate. In this case, it can be seen that the structure function measured by two jets is a weighted average

$$
F(x)=G(x)+\frac{4}{9}(Q(x)+\bar{Q}(x))
$$

where $G(x)$ is the gluon structure function and $Q(x)$ is the quark structure function averaged over all the quark species that contribute and $\bar{Q}(x)$ is the 


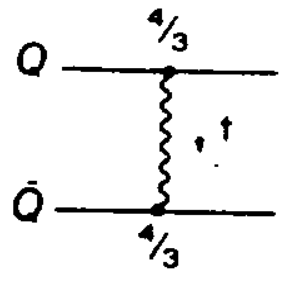

(a)

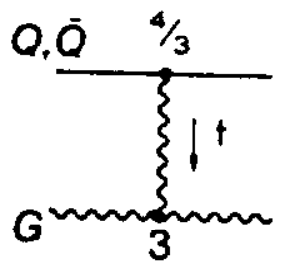

(b)

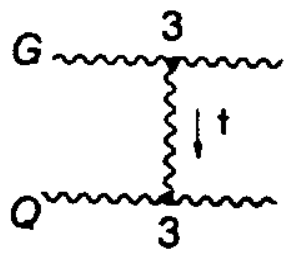

(c)

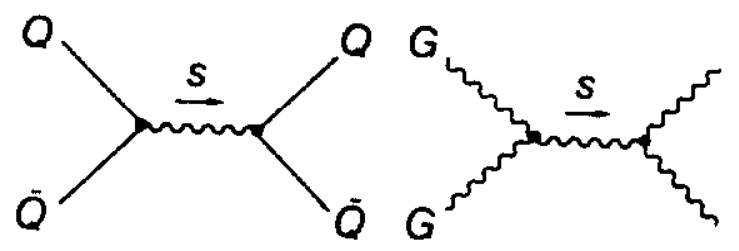

(d)

(e)

Figure 26: Born diagrams for 2 jet production

anti-quark structure function averaged over all the sea anti-quarks. The factor $4 / 9$ stems from the relative coupling strengths of the gluon and the quark. The averaging is valid if the angular distributions of the three $\hat{t}$ channel processes are similar. The angular distributions can be evaluated to leading log approximation in QCD [40]

$$
\frac{d \sigma}{d \cos \theta}=\frac{\pi \alpha_{0}^{2}}{8} \frac{\theta}{16} \frac{\left(3+\cos ^{2} \theta\right)^{3}}{\left(1-\cos ^{2} \theta\right)^{2}}
$$

for $\cos \theta \approx 1$ this expression reduces to

$$
\frac{d \sigma}{d \Omega}=\frac{9}{8} \frac{\alpha_{s}^{2}}{\sin ^{4}(\theta / 2)}
$$

which is just the Rutherford formula for Coulomb scattering for a $1 / r$ potential modified by some color factors. The angular distribution of two jets gives you information about the parton-parton potential at short distances. The exact expressions for $|A|^{2}$ for all the processes giving rise to two jets [41] are given in table (1). 


\begin{tabular}{|c|c|c|}
\hline Process & $|A|^{2}$ & $\theta=\pi / 2$ \\
\hline$q q^{\prime} \rightarrow q q^{\prime}$ & $\frac{4}{9} \frac{\hat{s}^{2}+\hat{u}^{2}}{\hat{t}^{2}}$ & 2.22 \\
\hline $99 \rightarrow 99$ & $\frac{4}{9}\left(\frac{\dot{s}^{2}+\dot{u}^{2}}{\hat{t}^{2}}+\frac{\dot{s}^{2}+\hat{t}^{2}}{\hat{u}^{2}}\right)-\frac{8}{27} \frac{\hat{s}^{2}}{\hat{u} \hat{t}}$ & 3.26 \\
\hline$q \bar{q} \rightarrow q^{\prime} \overline{q^{\prime}}$ & $\frac{4}{9} \frac{\hat{i}^{2}+\hat{u}^{2}}{\hat{s}^{2}}$ & 0.22 \\
\hline$q \bar{q} \rightarrow q \bar{q}$ & $\frac{4}{9}\left(\frac{\hat{s}^{2}+\hat{u}^{2}}{\hat{t}^{2}}+\frac{\hat{t}^{2}+\hat{u}^{2}}{\hat{j}^{2}}\right)-\frac{8}{27} \frac{\hat{u}^{2}}{\hat{s} \hat{t}}$ & 2.59 \\
\hline$q \bar{q} \rightarrow 9 g$ & $\frac{32}{27} \frac{\hat{t}^{2}+\hat{u}^{2}}{\hat{t} \hat{u}}-\frac{8}{3} \frac{\hat{t}^{2}+\hat{u}^{2}}{\hat{j}^{2}}$ & 1.04 \\
\hline $99 \rightarrow 9 q$ & $\frac{1}{6} \frac{\hat{t}^{2}+\hat{u}^{2}}{\hat{t} \hat{u}}-\frac{3}{8} \frac{\hat{t}^{2}+\hat{u}^{2}}{\hat{j}^{2}}$ & 0.15 \\
\hline$g q \rightarrow 9 q$ & $-\frac{1}{9} \frac{\dot{j}^{2}+\hat{u}^{2}}{j \hat{u}}+\frac{\dot{i}^{2}+\dot{s}^{2}}{\hat{t}^{2}}$ & 6.11 \\
\hline $9 g \rightarrow g g$ & $\frac{9}{2}\left(3-\frac{\hat{t} \hat{u}}{\hat{s}^{2}}-\frac{\hat{s} \hat{u}}{\hat{t}^{2}}-\frac{\hat{s} \hat{t}}{\hat{u}^{2}}\right)$ & 30.4 \\
\hline
\end{tabular}

Table 1: Two to Two parton subprocesses. The third column gives the value of $|A|^{2}$ at 80 degrees in the CM frame. $g g$ process is by far the most important. 


\subsection{Scale breaking}

\subsubsection{Evolution of structure functions}

At low $Q^{2}$, the nucleon behaves like a point particle. As the $Q^{2}$ of the probe increases, the point-like parton constituents manifest themselves. In the naive parton model, this results in perfect Bjorken scaling. A schematic of this behavior [37] is shown in figure (27). This picture is at odds with any interacting field theory of the nucleon (not necessarily QCD). Probing the nucleon at high $Q^{2}$ will reveal effects where quarks emit the field carriers (gluons in QCD) which in turn couple to quark-antiquark loops. The momentum fraction carried by valence quarks should decrease with $Q^{2}$ and the valence quark structure functions will change (evolve) with $Q^{2}$ such that functions of the type $x u(x)$ will increase in value at low $x$ as $Q^{2}$ increases. This is a direct result of the fact that as $Q^{2}$ increases, the probability that a valence quark emits a gluon, thereby reducing its $x$, increases. So a field theoretical description of the nucleon must lead to scale breaking. (See Figure (28))[37].

Neglecting mass effects, using renormalization group arguments, (similar in spirit to those first used by Weizsacker and Williams [38] in 1934 in connection with QED), Altarelli and Parisi [39] were able to write down equations governing the $Q^{2}$ evolution for the structure functions.

$$
\frac{d u\left(x, Q^{2}\right)}{d \ln Q^{2}}=\frac{\alpha_{s}\left(Q^{2}\right)}{2 \pi} \int_{\nu=x}^{y=1} u\left(y, Q^{2}\right) P_{Q Q}\left(\frac{x}{y}\right) \frac{d y}{y}
$$

where $u\left(x, Q^{2}\right)$ is the up quark structure function. The meaning of the above equation is fairly straightforward to appreciate.

$\left.\alpha_{s}\left(Q^{2}\right) P_{Q Q}(z) / 2 \pi\right) d \ln \left(Q^{2}\right) d z$ is the probability that a quark with momentum fraction $y$ will be observed in a state with momentum fraction $x$ when the $Q^{2}$ of the probe is increased fractionally by $d \ln Q^{2}$. The variable $z \equiv x / y$. This leads to a change in structure function at a momentum fraction $\mathrm{x}$ such that

$$
d u\left(x, Q^{2}\right) d x=\frac{\alpha_{s}\left(Q^{2}\right)}{2 \pi} P_{Q Q}(z) d \ln \left(Q^{2}\right) d z u\left(y, Q^{2}\right) d y
$$



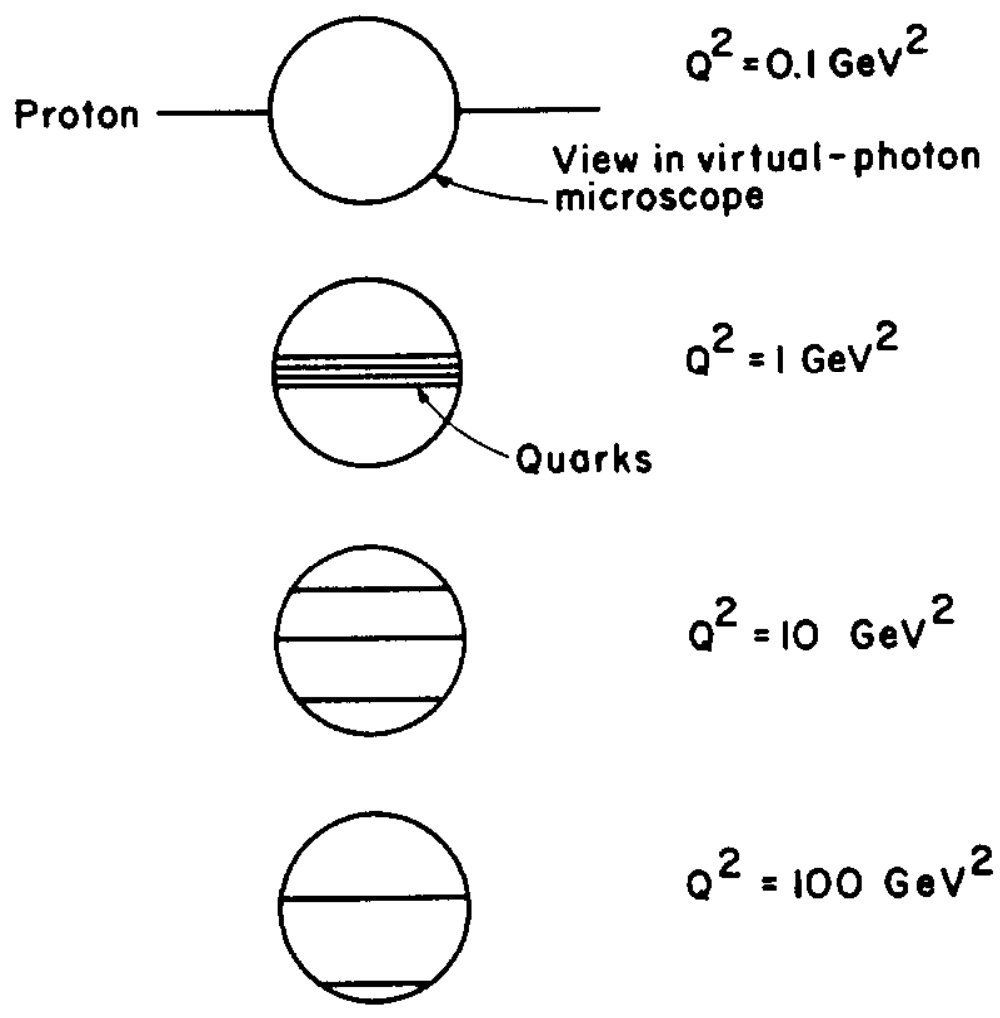

Figure 27: Picture of the nucleon in the naive parton model 


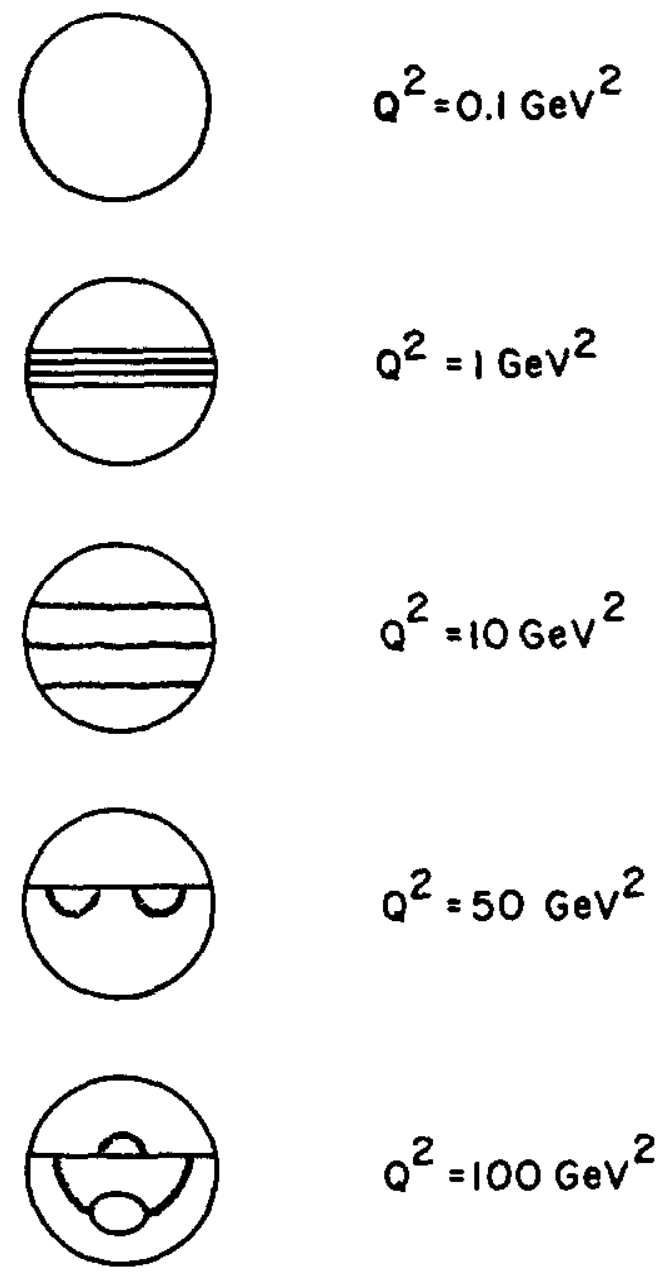

Figure 28: Picture of the nucleon in an interacting field theory 
Rearranging the differentials and integrating over $y$ leads to equation (47).

$$
P_{Q Q}(z)=\frac{4}{3} \frac{\left(1+z^{2}\right)}{(1-z)}
$$

The expression (49) for the 'splitting function' is very similar to one derived by Weizsacker and Williams for an electron radiating a photon. The factor $4 / 3$ is due to color. $\alpha_{\theta}\left(Q^{2}\right)$ is the QCD coupling constant which varies as a function of $Q^{2}$

$$
\alpha_{s}\left(Q^{2}\right)=\frac{\alpha_{s}\left(Q_{0}^{2}\right)}{1+B \alpha_{s}\left(Q_{0}^{2}\right) \ln \left(Q^{2} / Q_{0}^{2}\right)}=\frac{1}{B \ln \left(Q^{2} / \Lambda^{2}\right)}
$$

where $B=(33-2 f) / 12 \pi$ and $f$ is the number of flavors of quarks and $\Lambda^{2} \equiv Q_{0}^{2} \exp \left[-1 / B \alpha_{s}\left(Q_{0}^{2}\right)\right]$. So provided $f \leq 16, \alpha_{s}\left(Q^{2}\right)$ decreases as $Q^{2}$ increases and asymptotic freedom results. $Q_{0}^{2}$ is a reference value of $Q^{2}$ at which the structure functions are measured.

We are now in a position to take the structure functions obtained from deeply inelastic scattering [42] and evolve it to higher $Q^{2}$. This has been done by Eichten, Hinchliffe, Lane and Quigg [43] among others. Figures $(29,30,31)$ depict [41] the evolution of the various structure functions with $Q^{2}$. Figure (32) shows[41] the evolution of the bottom quark structure function of the proton. The heavy quark structure functions also evolve according to the Altarelli Parisi equations but one must take into account the effect of the masses of the heavy quarks and the associated production thresholds[44]. Figure (33) shows[41] the evolution of the fractional momentum of the proton carried by each parton as a function of $Q^{2}$. It can be shown using the Altarelli-Parisi equations that for 3 generations of quarks, the momentum fraction in gluons as $Q^{2} \rightarrow \infty$ tends to $8 / 17$ and each species of quark and anti-quark carries $3 / 68$ of the momentum of the proton. For a clear derivation of this result see the book by Quigg [37] page 241.

\subsection{Observation of jets}

Although there were hints of jet production at the ISR, jets in hadron collisions were unambiguously established as experimental fact by the UA2 [45] 


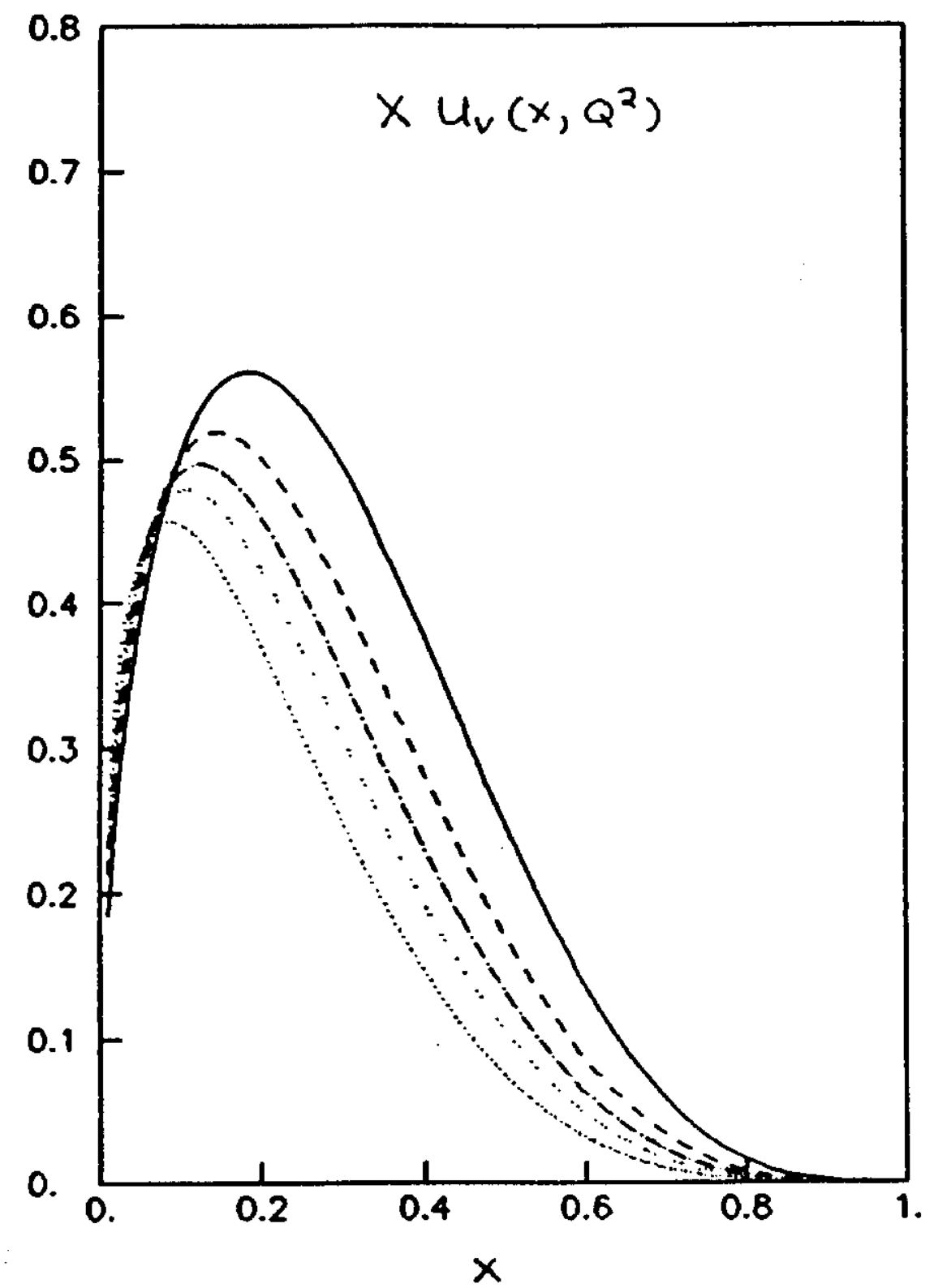

Figure 29: Valence up quark distribution of the proton $x u\left(x, Q^{2}\right)$ as a function of $x$ for various $Q^{2}$. The solid, dashed, dot-dashed, sparse dot, and dense dot lines correspond to $Q^{2}=10,10^{2}, 10^{3}, 10^{4}$ and $10^{6}(\mathrm{GeV})^{2}$ respectively. 


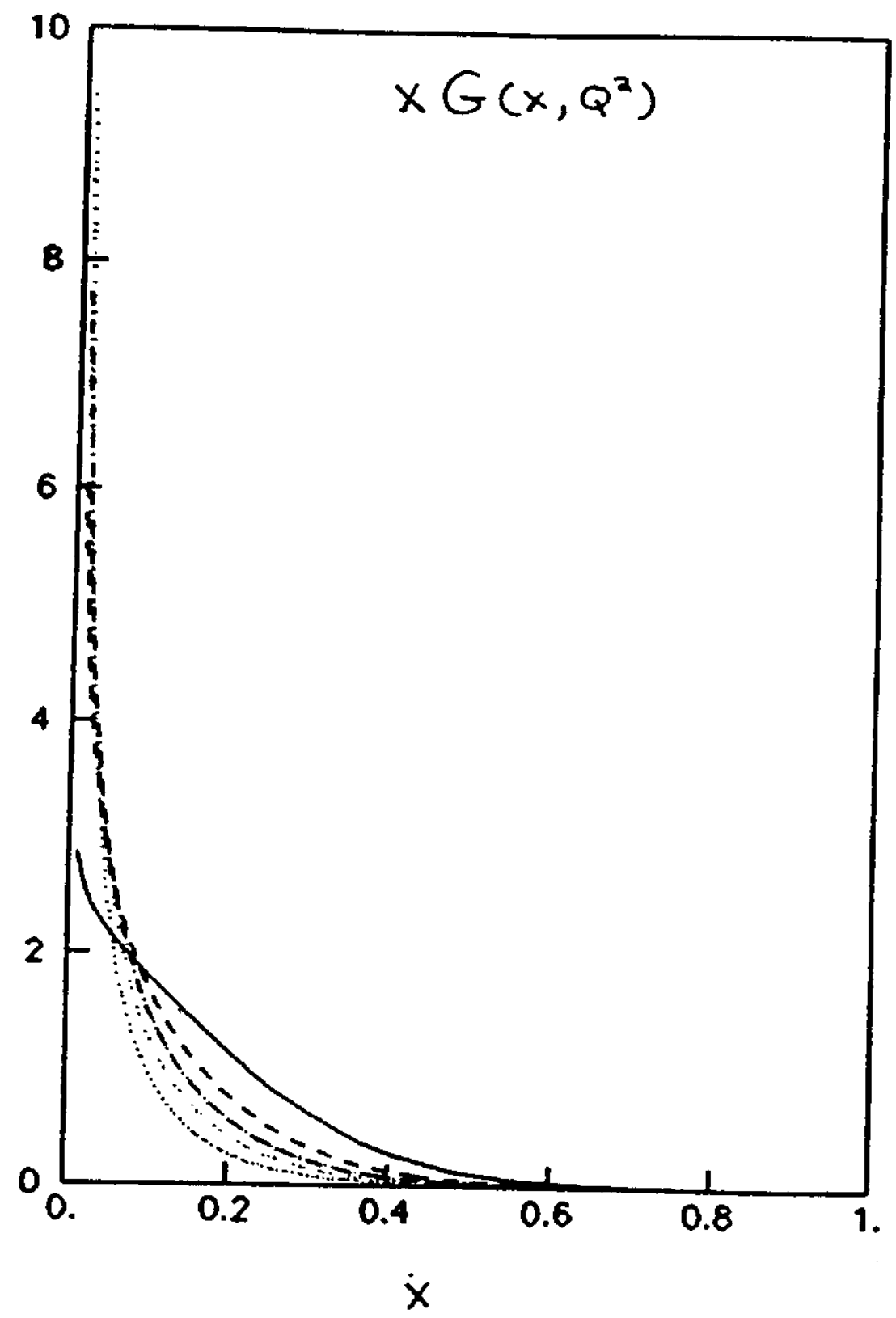

Figure 30: The gluon distribution of the proton, $x G\left(x, Q^{2}\right)$, as a function of $x$ for various $Q^{2}$. The solid, dashed, dot-dashed, sparse dot and dense dot lines correspond to $Q^{2}=10,10^{2}, 10^{3}, 10^{4}$ and $10^{6}(\mathrm{GeV})^{2}$ respectively. 


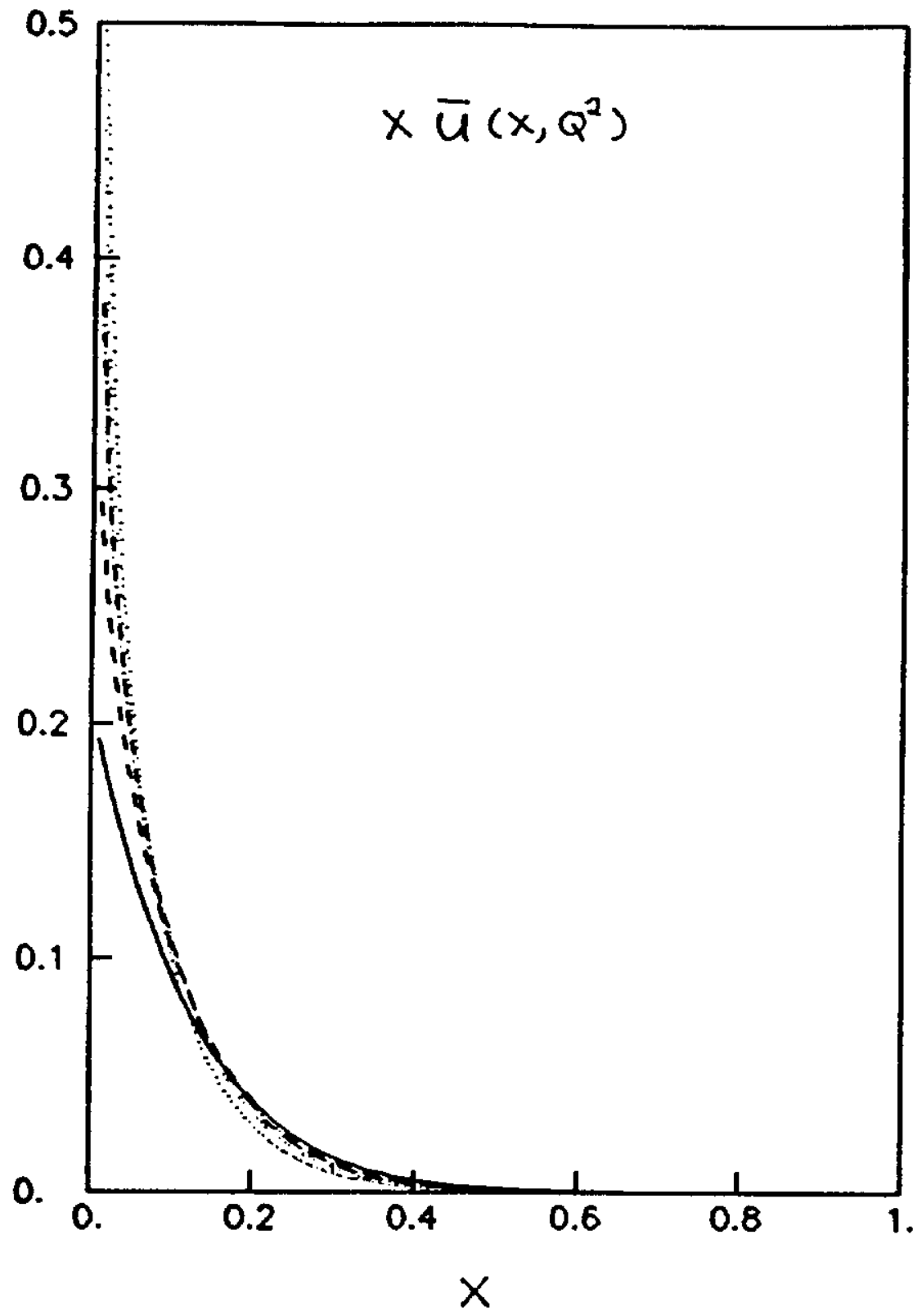

Figure 31: The up antiquark distribution of the proton, $x \bar{u}\left(x, Q^{2}\right)$, as a function of $x$ for various $Q^{2}$. The solid, dashed, dot-dashed, sparse dot and dense dot lines correspond to $Q^{2}=10,10^{2}, 10^{3}, 10^{4}$ and $10^{6}(\mathrm{GeV})^{2}$ respectively. 


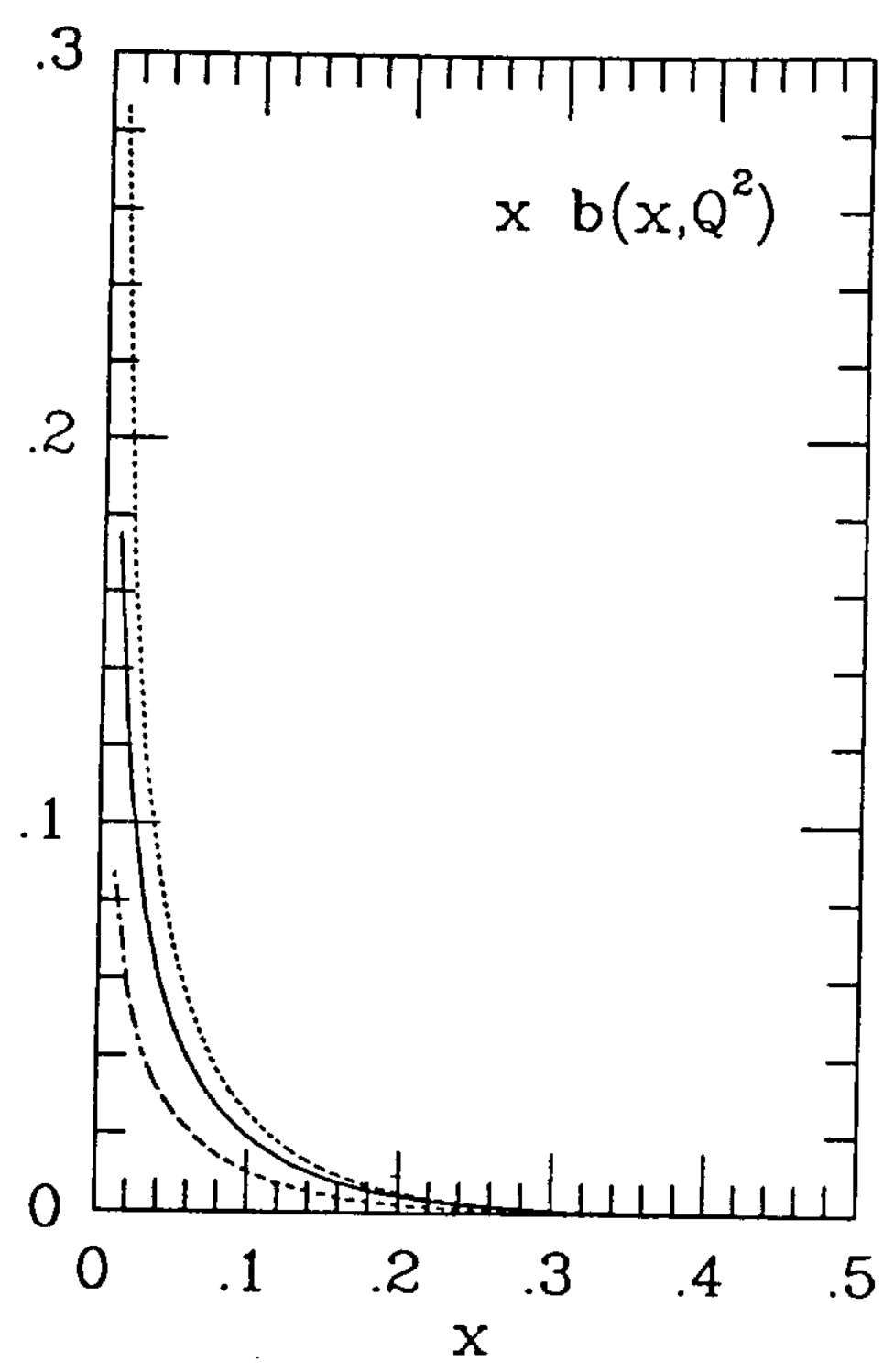

Figure 32: The bottom quark distribution of the proton, $x b\left(x, Q^{2}\right)$, as a function of $x$ for various $Q^{2}$. The dot-dashed,solid, and dotted lines correspond to $Q^{2}=10^{3}, 10^{4}$, and $10^{6}(\mathrm{GeV})^{2}$ respectively. 


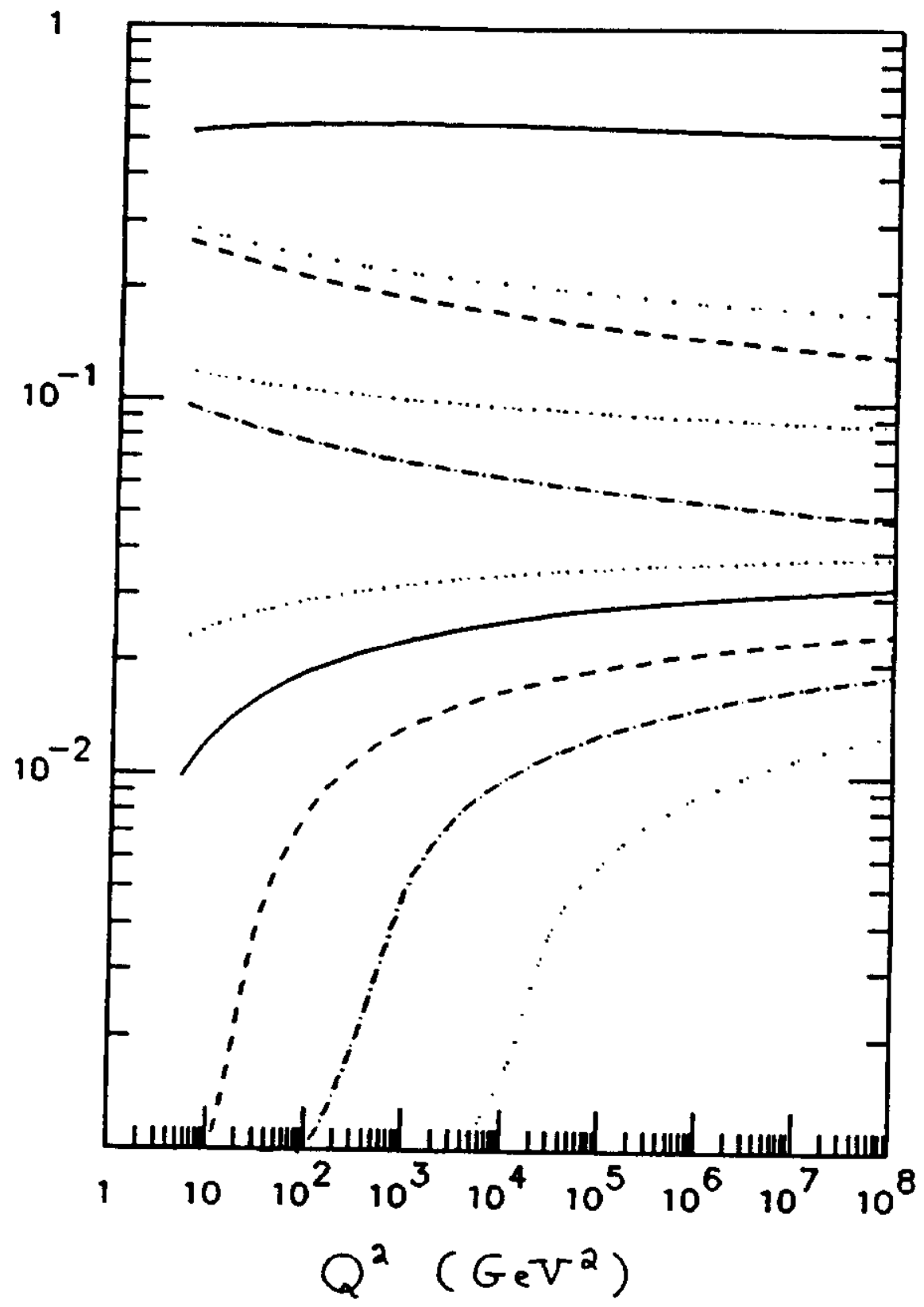

Figure 33: The fraction of the total momentum carried by each of the partons in the proton as a function of $Q^{2}$. from largest to smallest momentum fraction these partons are: gluon, up quark, up (valence only), down quark, down(valence only), antiup(or antidown) quark, strange quark, charm quark, bottom quark and top quark. 
and the UA1 [46] collaborations at the CERN $s \bar{p} p s$ collider. See Figure(34) for a typical jet event displayed as a LEGO plot. Figure (35) shows[51] the UA1 measurement of the inclusive jet cross section at $\eta=0$ as a function of $p_{t}$ for the two different values of $\sqrt{(s)}$. Because of the uncertainties in the measured jet energy scale, the measured cross sections at both values of $\sqrt{(s)}$ have a systematice error of $\pm 70 \%$. The QCD curves are renormalized upward by $50 \%$ to fit the data. It can be seen that the $p_{t}$ shape is well described by the data. The increase in jet cross section from $\sqrt{(s)}=546 \mathrm{GeV}$ to $\sqrt{(s)}=630 \mathrm{GeV}$ is well described by the QCD curves.

Figure (36) shows[51] the dimensionless cross section $p_{t}^{4} E d \sigma^{3} / d p^{3}$ as a function of the scaling variable $x_{T} \equiv p_{t} / \sqrt{(s)}$ The data are consistent with perfect $x_{T}$ scaling between the two values of total center of mass energy at CERN. To discern the effects due to QCD scale breaking, one needs to go to the Tevatron. Figure (37) shows the measurement of the effective structure function[2] measured by UA1 and UA2 versus equation (44). It can be seen that the effective structure function given by equation (44) describes the data well, and that the gluon contribution to two jet production is quite considerable at $s \bar{p} p s$ energies. References ([46]-[53]) and ([45], [54]-[61]) form a fairly exhaustive list of publications by UA1 and $\mathrm{UA2}$ respectively on jet production.

\subsubsection{Two jet angular distributions}

We saw that the equation (45) describing the 2 jet angular distribution is very similar to the Rutherford formula and behaved as

$$
\frac{d \sigma}{d \cos \theta} \approx \frac{A}{4 \sin ^{4}(\theta / 2)}=\frac{A}{(1-\cos \theta)^{2}}
$$

Define a variable $\chi \equiv(1+\cos \theta) /(1-\cos \theta)$. For exact Rutherford angular distribution, it is easy to show that $d \sigma / d \chi=$ constant). Figure (38) shows the UA1 data for the $\chi$ distribution of 2 jet events[62][50]. The data are in agreement with the prediction of leading order perturbative $Q C D$, that includes scale breaking effects. 


\section{Transverse energy deposition}

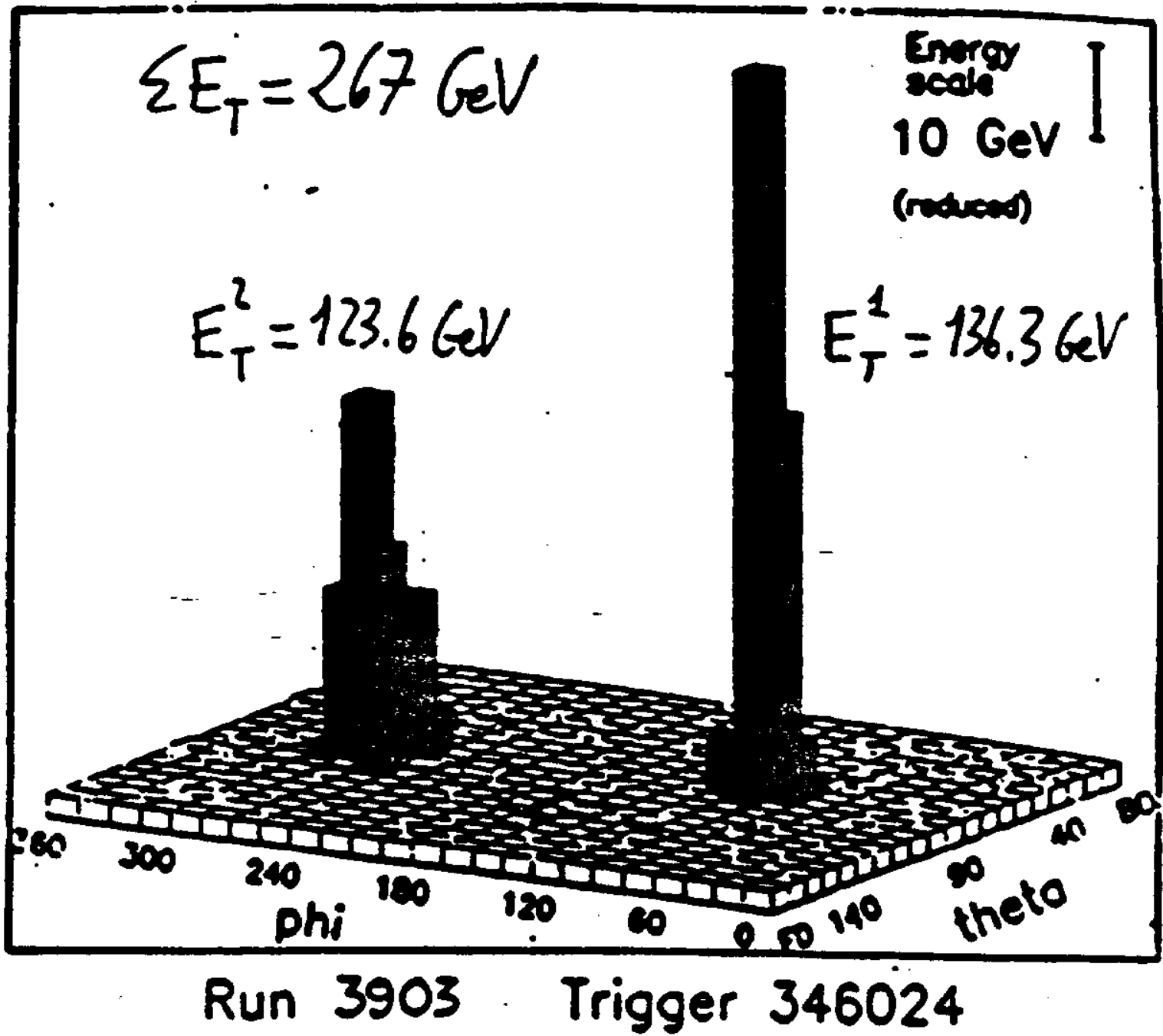

Figure 34: The highest energy 2jet event observed by the UA2 collaboration in the 1984 data. The height of each cell is proportional to the total energy deposition 


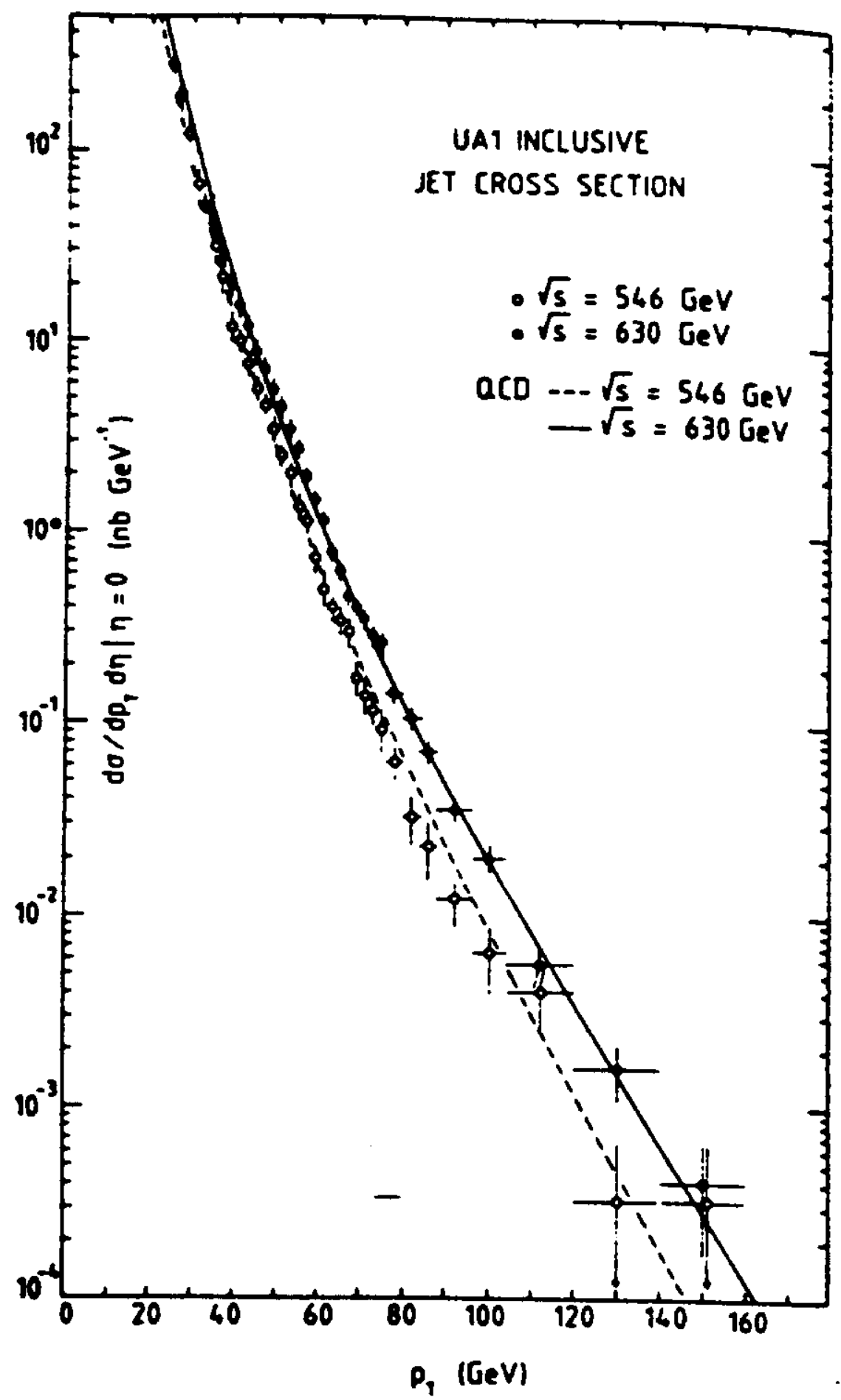

Figure 35: The inclusive jet cross section for the pseudorapidity interval $|\eta|<0.7$, as a function of the jet transverse momentum. The systematic error on both cross sections is $\pm 70 \%$. The curves are $Q C D$ calculations and are normalized upward by $\mathbf{5 0 \%}$ 


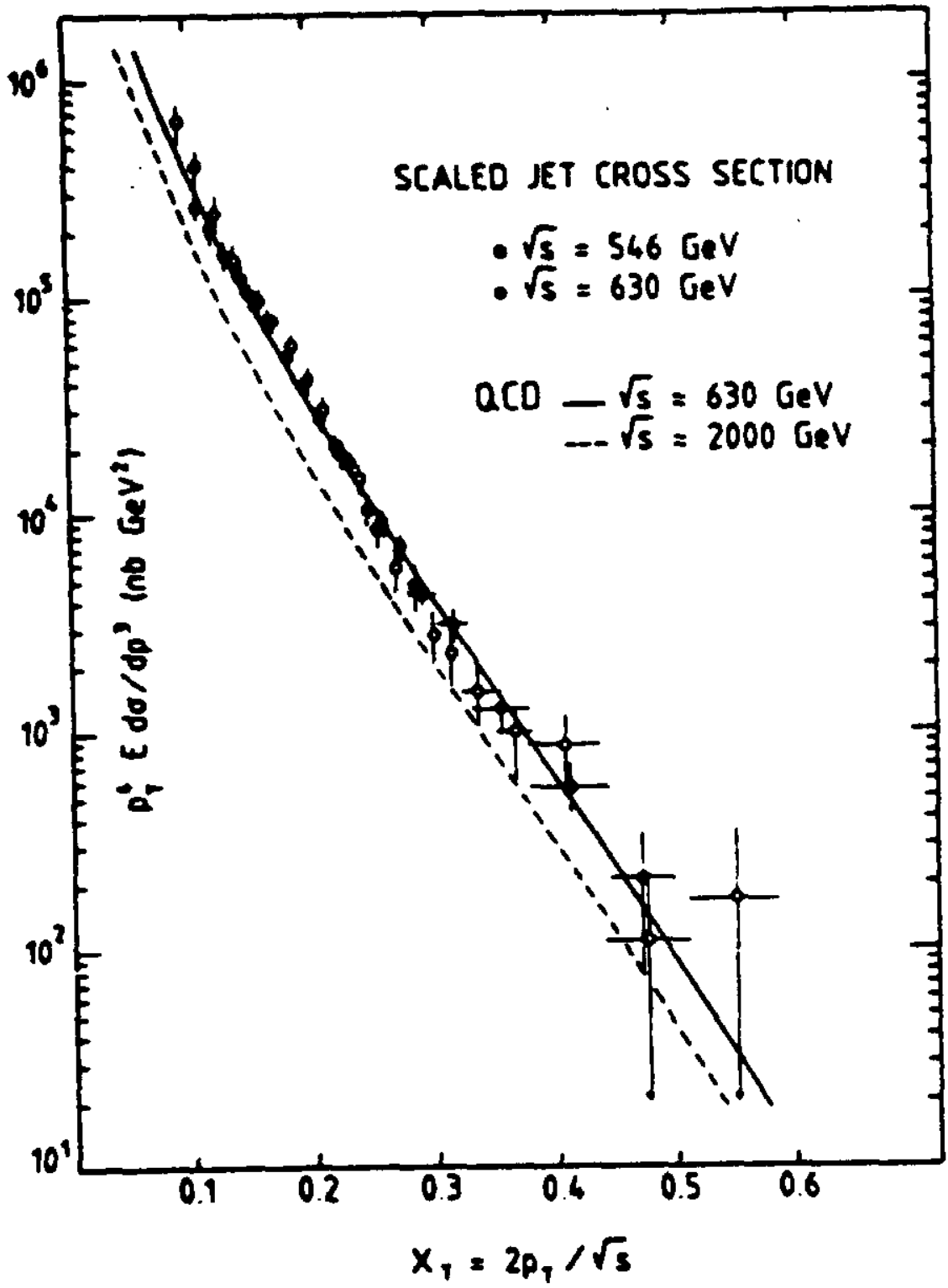

Figure 36: The scaled cross section as a function of the scaling variable $x_{T}$. The curves are QCD predictions for CMS energy $=630 \mathrm{GeV}$ (solid curve) and $2000 \mathrm{GeV}$ (dashed eurve). The predicted cross section at $546 \mathrm{GeV}$ is very similar to that at $630 \mathrm{GeV}$ 


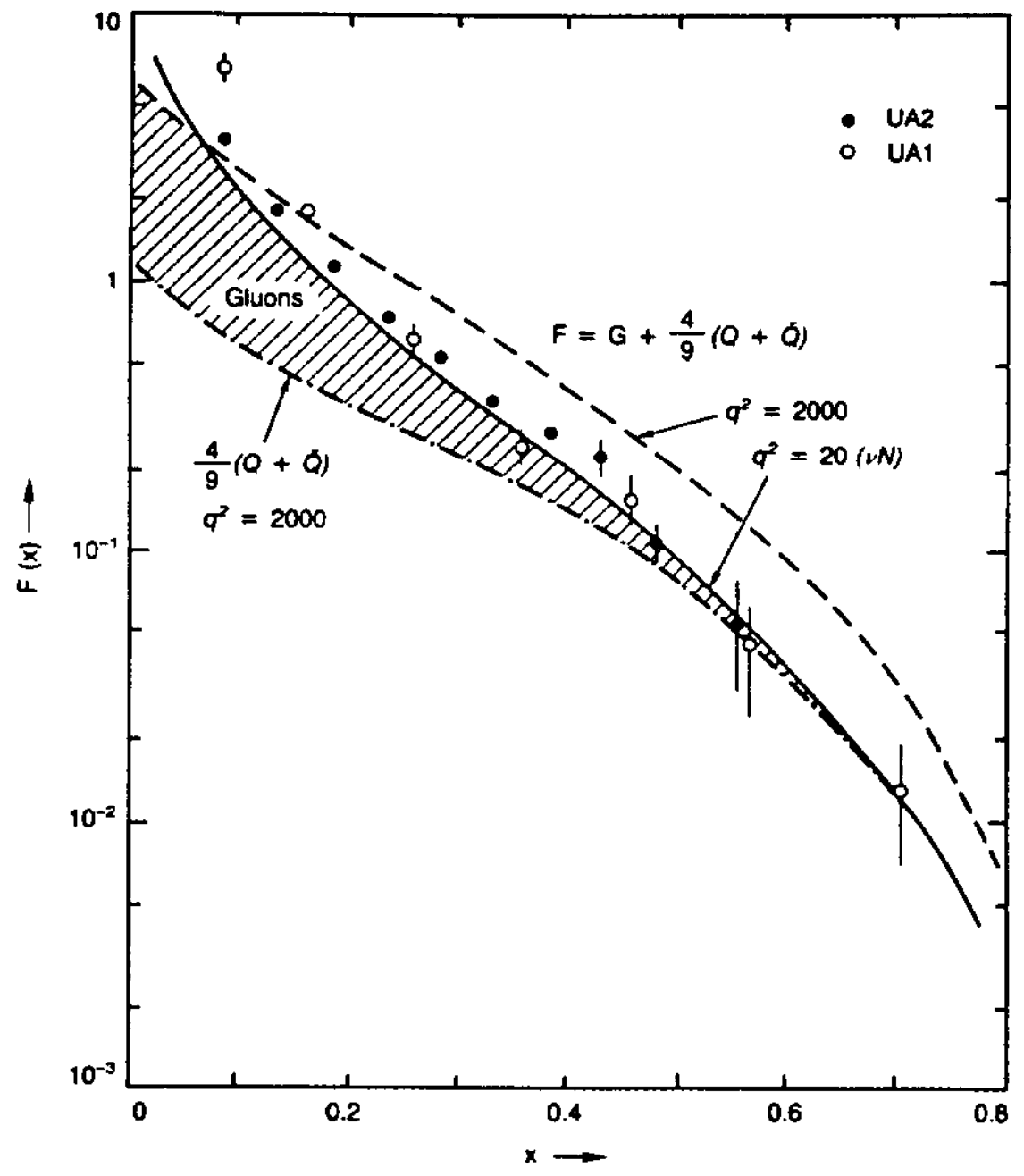

Figure 37: Points show structure function combination $G+4 / 9(Q+\bar{Q})$ deduced from UA1 and UA2 data. Dashed curve is same combination from neutrino- nucleon scattering data at $Q^{2}=20 \mathrm{GeV}^{2}$. Full curve is for $Q^{2}=2000 \mathrm{GeV}^{2}$. 

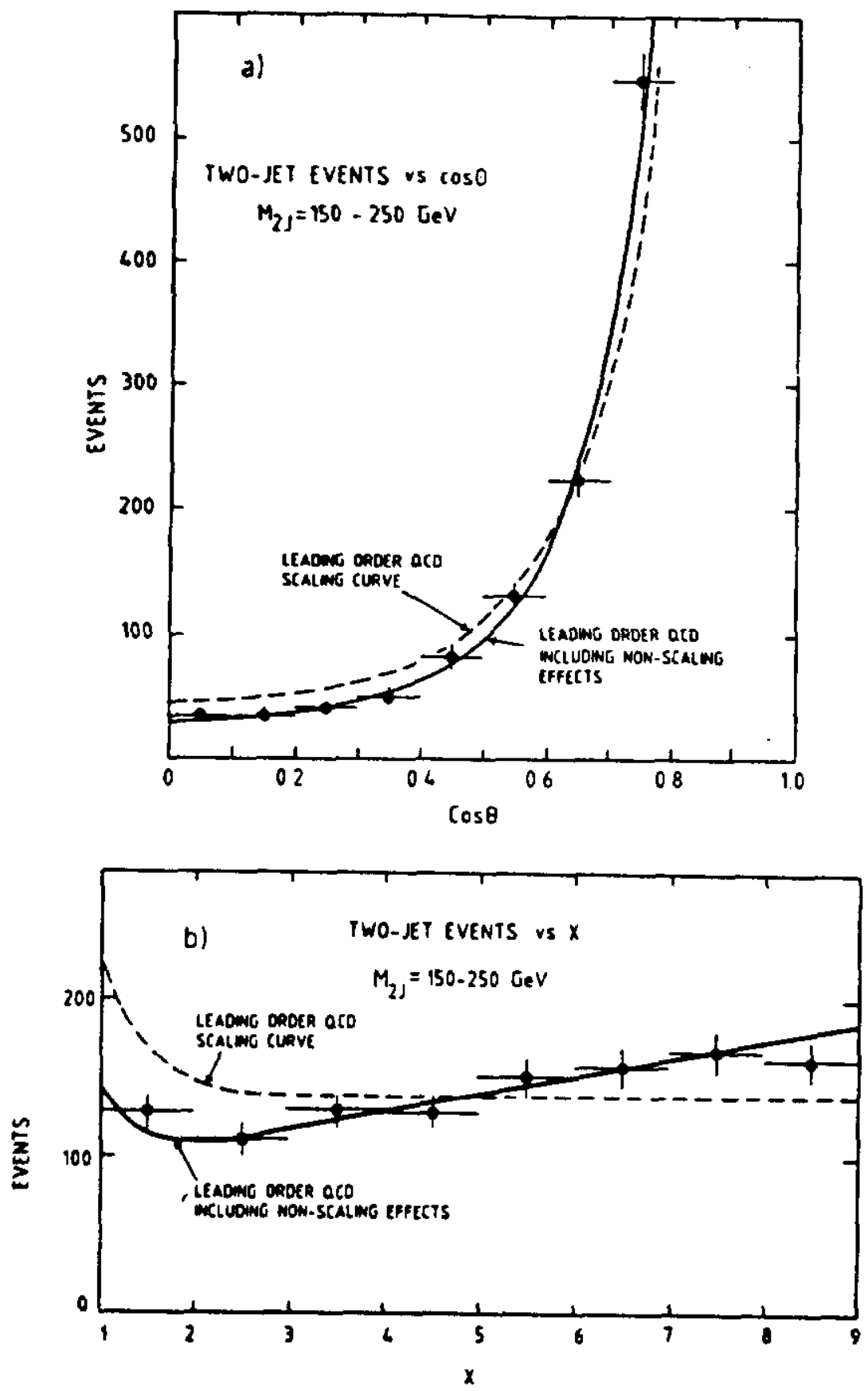

Figure 38: (a)The two-jet angular distribution plotted versus $\cos \theta$. (b) The two-jet angular distribution plotted versus $\chi$. The broken curves show leading order QCD predictions and the solid curves include scale-breaking corrections 


\subsubsection{Multijets}

It is possible for the collider partons to emit other partons before and/or after the primary collision. A multijet event is the result. The simplest of the multi-jet events is a 3 jet event where the additional jet can be produced by a gluon emitted prior to (initial state bremsstrahlung) and after (final state bremsstrahlung) the main collision. A typical 3 jet event observed by UA1 is shown in figure (39). The three jet variables used by UA1[50] are shown in figure(40). The angular distribution and other kinematic variables of the 3 jet events can be compared [50][61] with leading order QCD predictions[63]. There is in general good agreement as can be seen from figures $(41,42)$.

From the ratio of the 3 jet cross section to the 2 jet cross section, it should be possible to extract $\alpha_{s}$. However, uncertainties (infinities) in the QCD calculation when two of the 3 jets become collinear and ambiguities in defining a $Q^{2}$ scale to which the measured $\alpha_{s}$ should be assigned, make this method of determining $\alpha_{s}$ troublesome.

\subsubsection{Compositeness of quarks}

If the quark is a composite object, there will be an additional contact term interaction in quark-quark scattering which will manifest itself at sufficiently high $Q^{2}$ over and above single gluon exchange. This additional interaction could well show up at high enough jet $p_{t}$ 's as deviations from QCD predictions [64]. Using the present data, UAl have been able to set a limit of $\Lambda_{c}>400 \mathrm{GeV}$ on the quark compositeness mass scale[53][51] at the $95 \%$ confidence level.

\subsubsection{Jet fragmentation}

Fragmentation of partons into hadrons is perhaps one of the least understood features of QCD. Even though the primary scattering process is describable in terms of perturbative QCD, the hadronization chain contains very low $p_{t}$ hadrons ( $p_{t}$ measured respective to the parent parton direction) and the whole process is clearly a non-perturbative phenomenon involving final state interactions which have to conserve color and baryon number. 


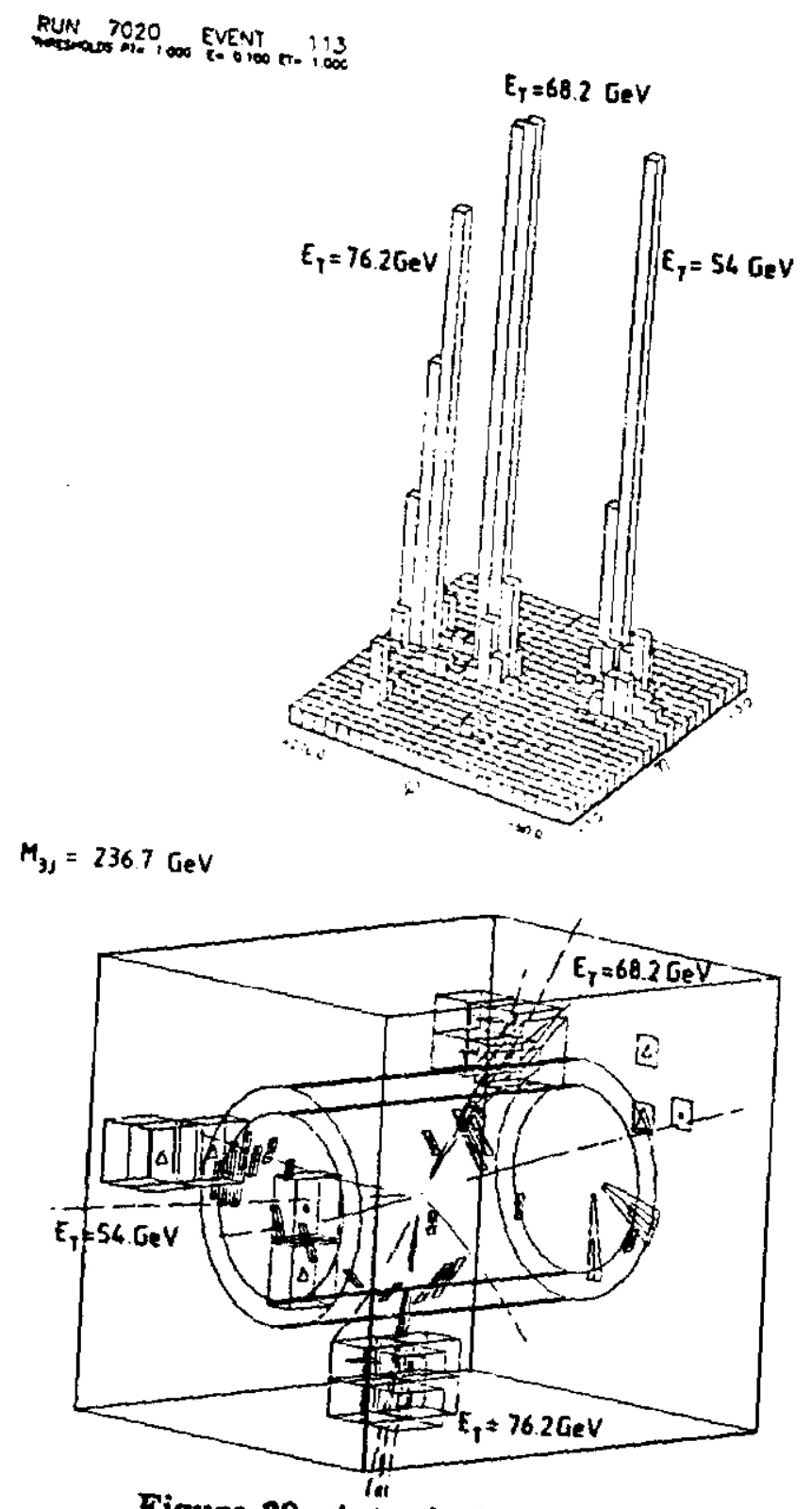

Figure 39: A typical 3 Jet event 


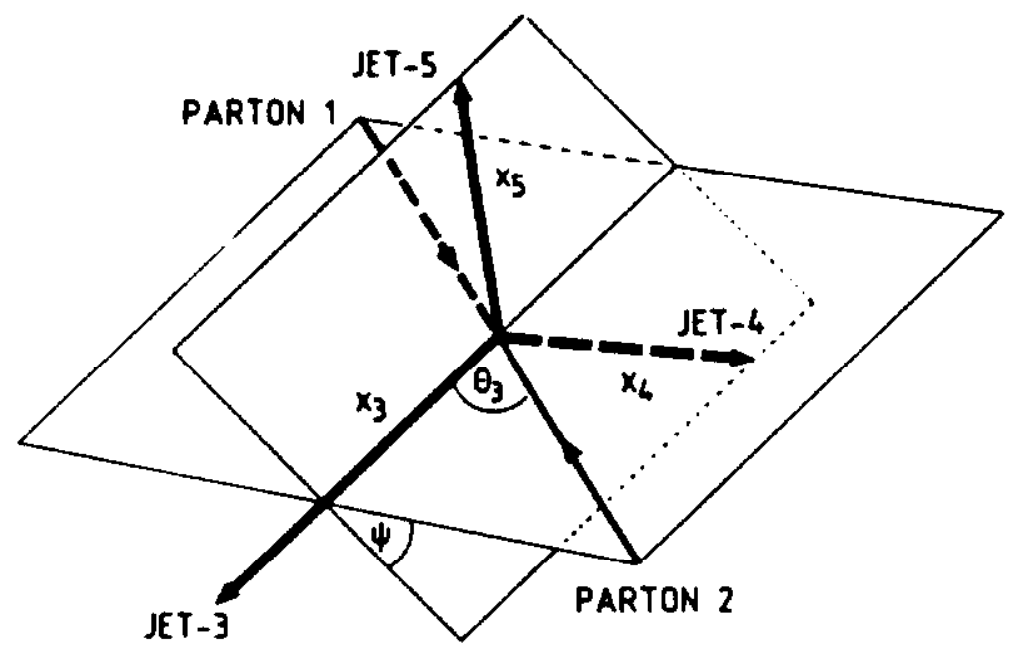

Figure 40: The definition of the three jet variables. Jet3 is the highest $E_{T}$ final state jet. Jet4 is the second highest and jet5 is the smallest $E_{T}$ jet

For this reason, totally independent fragmentation of the struck partons cannot occur. Attempts can be made however to parametrize the fragmentation process in terms of the variable $z \equiv p_{l}$ (jetaxis)/E(jet) /where $p_{l}$ (jetaxis) is the momentum of one of the charged hadrons along the jet axis |. The distribution function $D(z) \equiv 1 / N_{\text {jeto }}\left(d N_{c h} / d z\right)$ can be[36] used to characterize the process. Comparisons can be made of this quantity between collider jets and jets found in $e^{+} e^{-}$collisions. The disagreement between UAI data and the ISR and Petra data (which agree with each other) may well be due to systematic uncertainties in the algorithms involved in defining a jet and determining what tracks belong to a given jet. [48][52] Figure (43) shows such a comparison.

\subsection{QCD jets as source of background}

QCD jets are produced copiously at the collider. Because they are initiated to a large extent by gluons, the cross section increases with energy quite substantially. At $\sqrt{(s)}=630 \mathrm{GeV}$, UAl quote a differential cross sec- 


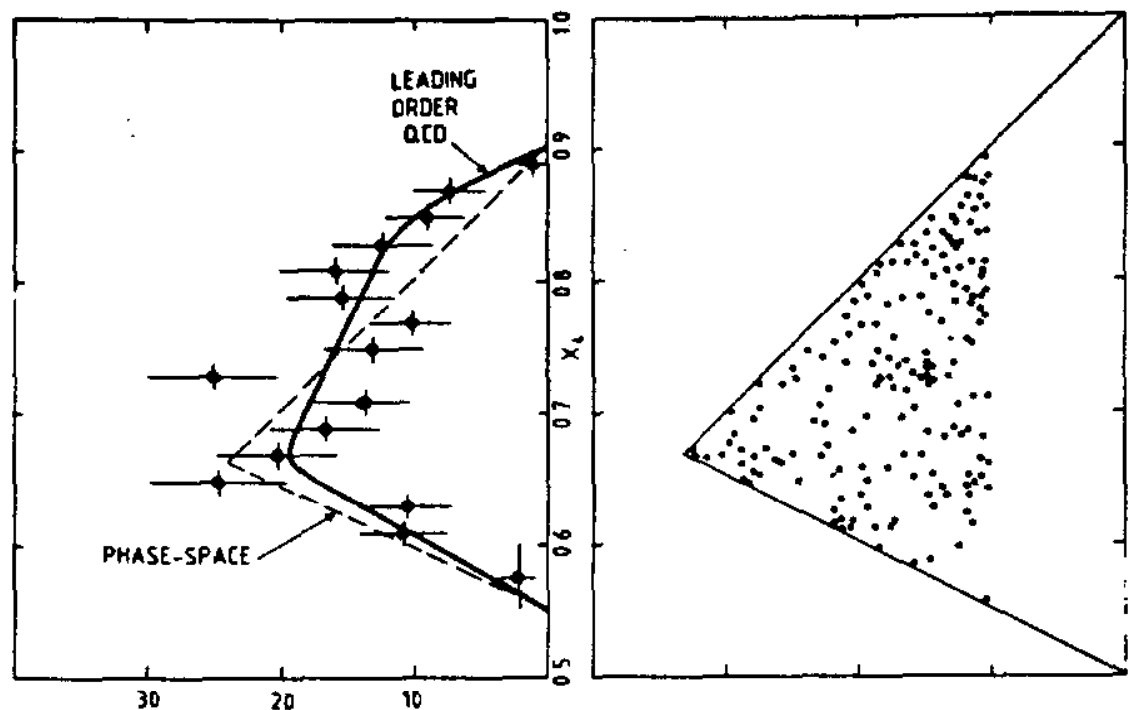

EVENTS PER 002 UNITS OF $x_{*}$

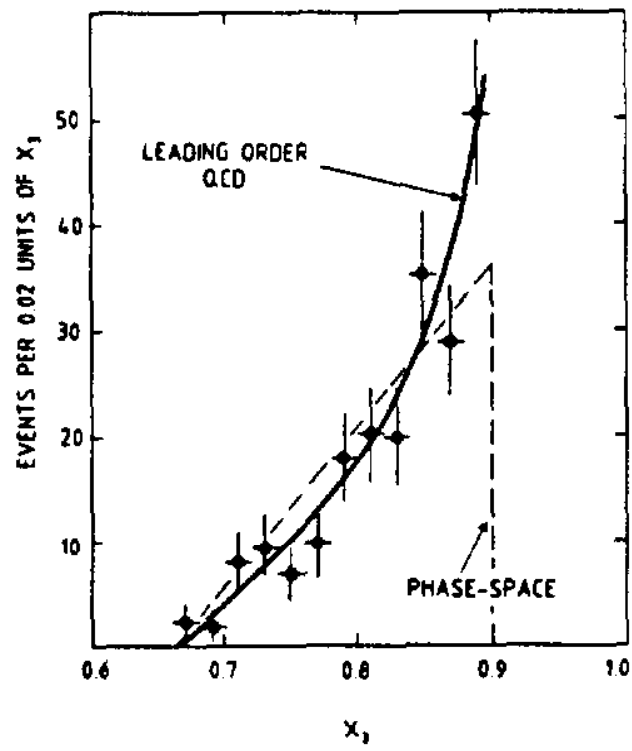

Figure 41: The Dalitz plot (x3 versus $\times 4$ ) for the three-jet sample. The solid curves represent the predictions of the leading order QCD formulae 


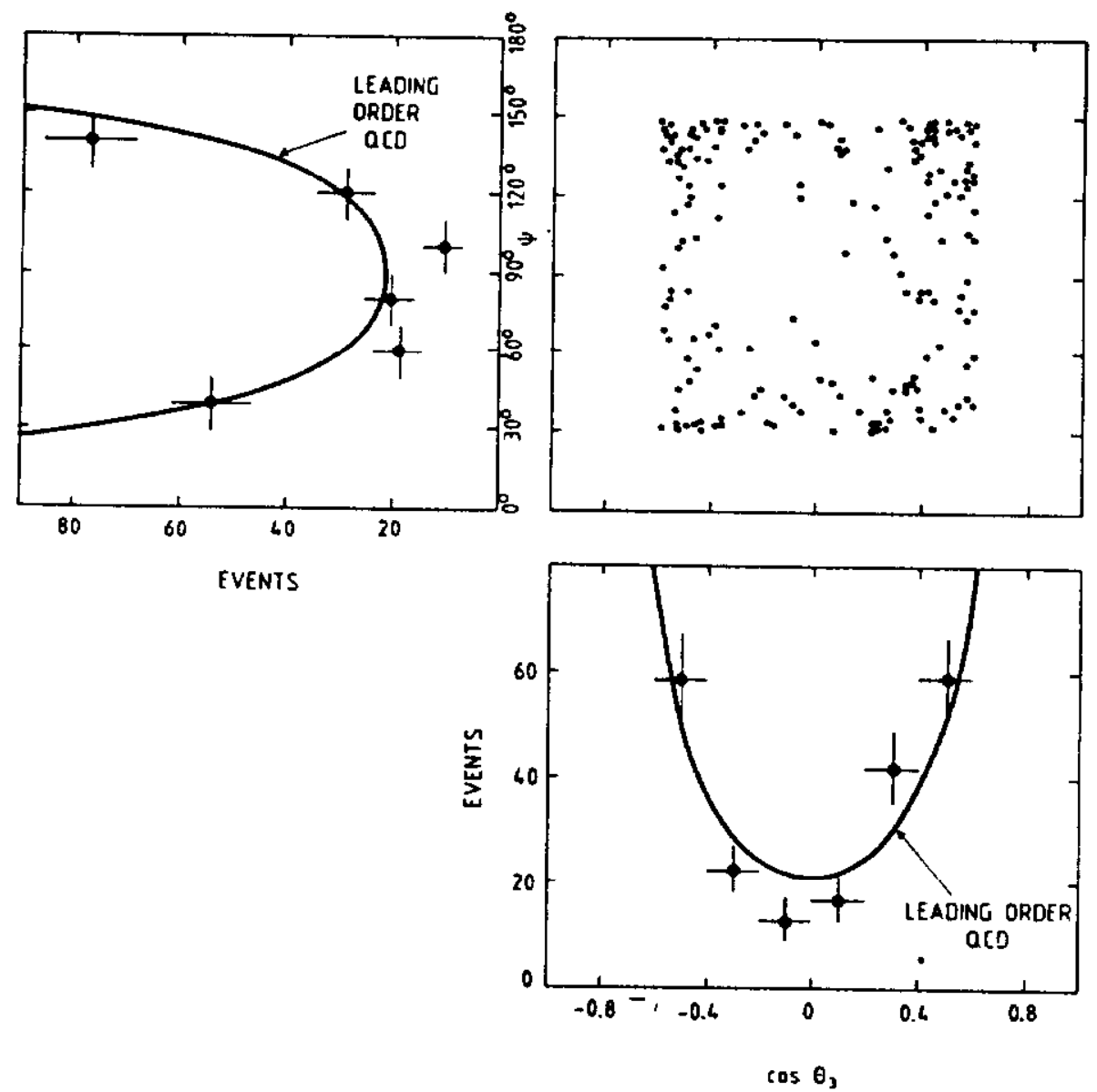

Figure 42: The three jet angular distribution: $\psi$ vs. $\cos \theta_{3}$. The theoretical curves are the predictions of the leading order QCD bremmstrahlung formulae 


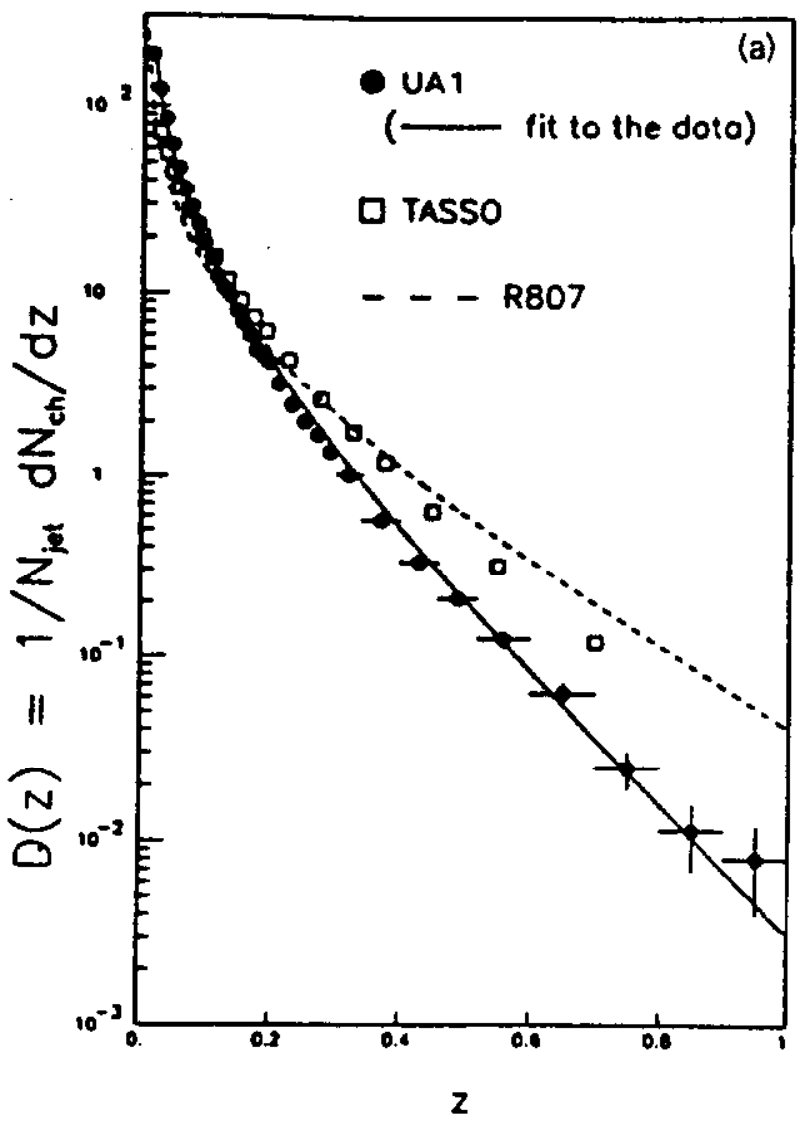

Figure 43: Comparison of fragmentation functions

tion $d \sigma / d p_{t} d \eta|\eta|<0.7$ of $15.2 \pm 2.2 n b / G e V$ at a $p_{t}$ of $41 \mathrm{GeV}$. This is a substantial cross section and any miss-measurement or fluctuation in the fragmentation topology of the jets can cause a feed down from the QCD jet channel into a channel where one is looking for a sare event. A case in point is when one of the jets fragments with low multiplicity and mimics an electron (a charged pion with an overlapping $\pi^{0}$ can do this). The other jet produces some missing $p_{t}$ by being badly measured. This event can end up as a background to the $W$ signal, although it is easily removed by demanding that the missing $p_{t}$ be not collinear with one of the jets. A more insidious case occurs for a 3 jet event where 2 of the 3 jets are badly measured (due to resolution fuctuations). In this case, the missing $p_{t}$ will point along a direction not collinear with any of the 3 jets. This type of topology is a signal for many an exotic particle search. It suffices to observe here that understanding the QCD jet processes is extremely important at the Tevatron, not only as a physics end in itself, but also to aid in searches for new phenomena. 


\section{W/Z Physics}

\subsection{Need for the existence of the $W$}

Without the $\mathrm{W}$, processes such as $\nu_{\mu} e^{-} \rightarrow \mu^{-} \nu_{e}$ (inverse muon decay) proceed via the four fermion contact interaction (see Figure (44). The $S$ wave amplitude for the above process can be written as

$$
M_{0}=\frac{G_{F} s}{\pi \sqrt{(2)}}\left[1-\frac{\left(\mu^{2}-m^{2}\right)}{s}\right] \approx \frac{G_{F} s}{\pi \sqrt{(2)}}
$$

where $G_{F}$ is the Fermi Constant, $s$ is the center of mass energy squared and $\mu, m$ are the muon and electron masses. Unitarity demands that $M_{0}<1$

which implies that $\sqrt{(s)}<617 \mathrm{GeV}$. So for the above reaction, unitarity will be violated above a center of mass energy of $617 \mathrm{GeV}[37]$. The solution is to introduce a heavy boson that carries a charge \pm 1 . It must be massive in order to reproduce the short range nature of the weak interaction, much in the spirit of the Yukawa potential for the weak interaction. See Figure (45). Now

$$
M_{0}=\frac{G_{F} M_{W}^{2}}{\pi \sqrt{(2)}} \log \left(1+\frac{s}{M_{W}^{2}}\right)
$$

This implies that for a $W$ mass of $\approx 100 \mathrm{GeV} / c^{2}$, unitarity is respected up to a center of mass energy of $3.5 \times 10^{23} \mathrm{GeV}$. One cannot, however, conquer this violation by introducing higher order terms. The above theory is nonrenormalizable. Presumably, by considering $\nu_{\mu} e^{-}$elastic scattering, one can make similar arguments for the existence of the $\mathrm{Z}$ boson. After much ingenious work, notably by Glashow, Salam, Weinberg, 't Hooft, Veltman and Ben Lee ([65]-[70]), the (SU(2)xU(1)) gauge theory emerged as the leading candidate for unifying electromagnetic and weak interactions.

The discovery of neutral currents at CERN ([71]) led to intense interest in the electroweak theory. A series of experiments at CERN, Fermilab and SLAC [72]) confirmed the electroweak theory, and established a value for the Weinberg angle. This led to predictions for the masses of the $W$ and $Z$ bosons of $83 \mathrm{GeV} / \mathrm{c}^{2}$ and $95 \mathrm{GeV} / \mathrm{c}^{2}$ respectively. The SU(2)xU(1) electroweak theory became known as the standard model. This further led 
to a proposal by Rubbia [73] to upgrade the CERN SPS to do $\bar{p} p$ collisions. The invention of stochastic cooling by van der Meer [74] made possible the CERN $s \bar{p} p s$ collider. The spectacular discovery of the $\mathrm{W}$ and the $\mathrm{Z}$ bosons by the UA1 and UA2 experiments [75]at CERN lent further support to the standard model. The standard model, as it stands now, demands the following picture with 3 generations of quarks and leptons.

$$
\begin{array}{cccc}
\left(\begin{array}{l}
u \\
d
\end{array}\right)_{L} & \left(\begin{array}{c}
c \\
s
\end{array}\right)_{L} & \left(\begin{array}{c}
t \\
b
\end{array}\right)_{L} & \text { quarks,3colors. } \\
u_{R}, d_{R} & c_{R}, s_{R} & t_{R}, b_{R} \\
\left(\begin{array}{c}
\nu_{e} \\
e
\end{array}\right)_{L} & \left(\begin{array}{c}
\nu_{\mu} \\
\mu
\end{array}\right)_{L} & \left(\begin{array}{c}
\nu_{\tau} \\
\tau
\end{array}\right)_{L} & \text { Leptons. } \\
e_{R} & \mu_{R} & \tau_{R}
\end{array}
$$

where the subscripts $L, R$ stand for left and right handed respectively. Each particle in the above table has its anti-particle. The gauge bosons $W, Z, \gamma$ and the 8 colored gluons complete the standard model. Of the particles listed above, the top quark alone remains undiscovered. Though the $\nu_{r}$ has not been directly observed, its existence is not seriously doubted at present. In this chapter, we will deal with

- $\mathrm{W} / \mathrm{Z}$ cross sections. Derivation of the parton fusion model.

- Observation of $\mathrm{W}$ and $\mathrm{Z}$ bosons. Production properties.

- Standard Model parameters. Decay Properties.

- Limits on the number of neutrino species

- High $p_{t}$ jets produced with W's (?) 


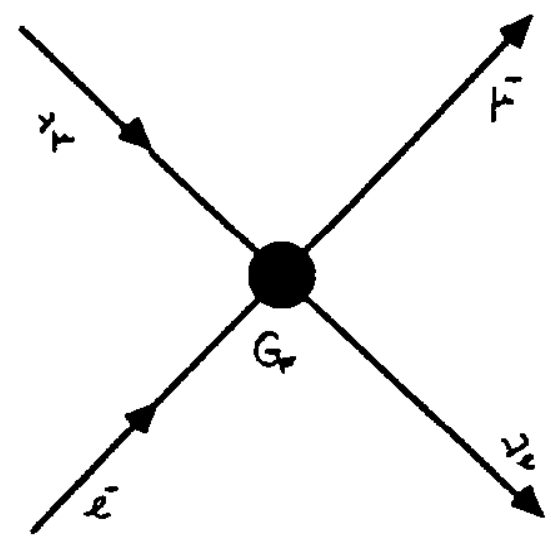

Figure 44: Four fermion contact interaction

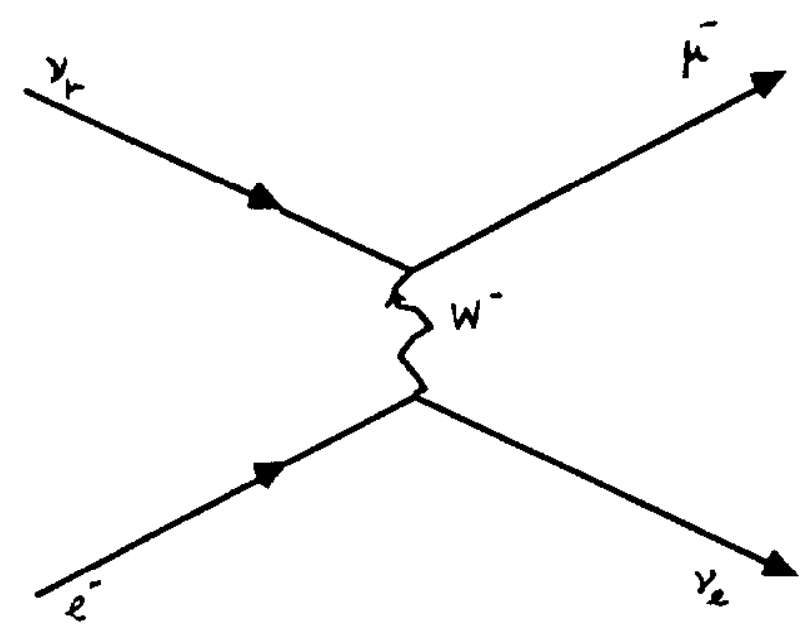

Figure 45: Born diagram with $W$ propagator 


\subsection{W/Z production cross sections}

\subsubsection{Derivation of the parton fusion model}

Using equation (27), we see that the $\mathrm{W}$ production cross section can be written

$$
\frac{d^{2} \sigma_{W}}{d x_{a} d x_{b}}=\sum_{i, j} \frac{1}{1+\delta_{i j}}\left[f_{i}^{(a)}\left(x_{a}, Q^{2}\right) f_{j}^{(b)}\left(x_{b}, Q^{2}\right)+i \leftrightarrow j\right] \hat{\sigma}(i j \rightarrow W)
$$

where $x_{a} x_{b}=\tau=M_{W}^{2} / s$. The structure functions depend on $Q^{2}$. There exists some ambiguity as to what the exact value of $Q^{2}$ should be. $Q^{2} \approx$ $M_{W}^{2}$ is perhaps a natural choice. In what follows, we will suppress the $Q^{2}$ dependence of the structure functions for purposes of brevity.

\subsubsection{Dexivation of the Breit- Wigner resonance formula}

In equation(54), $\hat{\sigma}(i j \rightarrow W)$ is a function of $\hat{s}$ and for a mass and total width of the W of $M_{W}, \Gamma_{W}$, can be written in the familiar Breit-Wigner form

$$
\hat{\sigma}(i j \rightarrow W)=\frac{4 \pi \lambda^{2}(2 l+1) \Gamma_{W \rightarrow i j} \Gamma_{W}}{\left(2 s_{i}+1\right)\left(2 s_{j}+1\right)\left[\left(M_{W}-E\right)^{2}+\left(\Gamma_{W}^{2} / 4\right)\right]}
$$

where $E=\sqrt{\hat{s}}, l$ is the spin of the W, $s_{i, j}$ are the spins of the fusing partons and $\Gamma_{W \rightarrow i j}$ is the partial width of $\mathrm{W}$ decay to the partons $i, j$. The above form for the Breit-Wigner is non-relativistic and is easily derived from the partial wave expansion derived for elastic scattering(4)

$$
f_{l}=\left[\frac{\eta_{l} e^{2 i \delta_{l}}-1}{2 i}\right]
$$

$\delta_{l}$ is a function of the center of mass energy $E[2]$. When $\delta_{l}=\pi / 2$, the partial wave $l$ is said to resonate. If $\eta_{l}=1$, for all $l$, we are operating below the inelastic threshold and the total cross section is the same as the elastic cross section. We make this assumption for the sake of simplicity, and will modify the Breit-Wigner formula accordingly to take into account the effect of inelasticity. Then, dropping the subscript $l$,

$$
f=\frac{e^{i \delta}\left[e^{i \delta}-e^{-i \delta}\right]}{2 i}=e^{i \delta} \sin \delta=\frac{1}{\cot \delta-i}
$$


Since $\delta \approx \pi / 2, \cot \delta \approx 0 . E=M_{W}$ is the value at which $\delta=\pi / 2$. Taylor expanding about this point,

$$
\cot \delta(E)=\cot \delta\left(M_{W}\right)+\left(E-M_{W}\right)\left[\frac{d}{d E} \cot \delta(E)\right]_{E=M_{W}}=-\left(E-M_{W}\right) \frac{2}{\Gamma}
$$

where we have defined $2 / \Gamma=-[\operatorname{dcot} \delta(E) / d E]_{E=M_{W}}$. If, $E-M_{W} \approx \Gamma \ll$ $M_{W}$, we may neglect further terms, giving

$$
f(E)=\frac{1}{\cot \delta-i}=\frac{\Gamma / 2}{\left(M_{W}-E\right)-i \Gamma / 2}
$$

So using equation (5), we get

$$
\sigma_{e l}(E)=\frac{4 \pi \lambda^{2}(2 l+1) \Gamma^{2} / 4}{\left(E-M_{W}\right)^{2}+\Gamma^{2} / 4}
$$

The above formula is for elastic scattering of spinless particles. When there is inelasticity, the term $\Gamma^{2}$ in the numerator is modified to $\Gamma_{W \rightarrow i j} \Gamma_{W}$ for the formation and decay processes. For spinning partons, we have to average over the initial spin states. The resonating partial wave $l$ is clearly the spin of the $\mathrm{W},(l=1)$, yielding equation (55).

Equation (54) can be re-written in terms of variables $x_{a}$ and $\tau$.

$$
\frac{x_{a} d^{2} \sigma_{W}}{d x_{a} d \tau}=\sum_{i, j} \frac{1}{1+\delta_{i j}}\left[f_{i}^{(a)}\left(x_{a}\right) f_{j}^{(b)}\left(\tau / x_{a}\right)+i \leftrightarrow j\right] \hat{\sigma}(i j \rightarrow W)
$$

If one assumes that the Breit-Wigner is narrow, one can integrate over it while assuming that the structure function $f\left(\tau / x_{a}\right)$ does not vary appreciably over its width. Then,

$$
\frac{x_{a} d \sigma_{W}}{d x_{a}}=\sum_{i, j} \frac{1}{1+\delta_{i j}}\left[f_{i}^{(a)}\left(x_{a}\right) f_{j}^{(b)}\left(\tau / x_{a}\right)+i \leftrightarrow j\right] \int \hat{\sigma}(i j \rightarrow W) d \tau
$$

Using $\tau=E^{2} / s, \lambda=2 / M_{W}$, we can integrate over the Breit-Wigner (Change the variable of integration from $\tau$ to $\tan \theta=2\left(M_{W}-E\right) / \Gamma$ ) to yield

$$
\int \hat{\sigma}(i j \rightarrow W) d \tau=\frac{16 \pi^{2}(2 l+1) \Gamma_{W \rightarrow i j}}{M_{W} s\left(2 s_{i}+1\right)\left(2 s_{j}+1\right)}
$$


This then yields,

$$
\sigma_{W}=\frac{16 \pi^{2}(2 l+1)}{M_{W}^{3}\left(2 s_{i}+1\right)\left(2 s_{j}+1\right)} \sum_{i j} \frac{\Gamma_{W \rightarrow i j}}{1+\delta_{i j}} \tau \int_{z_{a}=\tau}^{x_{a}=1}\left[f_{i}^{a}\left(x_{a}\right) f_{j}^{b}\left(\frac{\tau}{x_{a}}\right)+i \leftrightarrow j\right] \frac{d x_{a}}{x_{a}}
$$

This is essentially the same as the gluon fusion model for $\eta_{c}$ production first written down by Einhorn and Ellis[76]. Since all the fusing partons to date considered have spin multiplicity 2 , (gluons have only two states of polarization, being massless), we can write the above equation without loss of generality as

$$
\sigma_{W}=\frac{4 \pi^{2}(2 l+1)}{M_{W}^{3}} \sum_{i j} \frac{\Gamma_{W \rightarrow i j}}{1+\delta_{i j}} \tau \int_{x_{a}=\tau}^{x_{a}=1}\left[f_{i}^{a}\left(x_{a}\right) f_{j}^{b}\left(\frac{\tau}{x_{a}}\right)+i \leftrightarrow j\right] \frac{d x_{a}}{x_{a}}
$$

So far we have totally ignored color. The structure functions are integrated over all quark colors. There are in all 9 color combinations of $i j$, of which only 3 contribute to producing colorless W's. This gives a factor of $1 / 3$. Also $\Gamma(W \rightarrow i j)$ refers to $W$ coupling to one of these 3 combinations that contribute in the above formula. If we use the physical width, then we get another factor of $1 / 3$ giving an overall factor of $1 / 9$ yielding

$$
\sigma_{W}=\frac{4 \pi^{2}}{3 M_{W}^{3}} \sum_{i j} \frac{\Gamma_{W \rightarrow i j}}{1+\delta_{i j}} \tau \int_{x_{a}=\tau}^{x_{a}=1}\left[f_{i}^{a}\left(x_{a}\right) f_{j}^{b}\left(\frac{\tau}{x_{a}}\right)+i \leftrightarrow j\right] \frac{d x_{a}}{x_{a}}
$$

Using the fact that

$\Gamma(W \rightarrow u \bar{d})=\left(G_{F} M_{W}^{3}\right) \cos ^{2} \theta_{c} /(2 \pi \sqrt{2})$ where $\theta_{c}$ is the Cabibbo angle $\left(\cos \theta_{c}=0.9737 \pm 0.0025\right)[77]$, we can re-write the above equation as,

$\sigma_{W+}=\frac{\pi \sqrt{2} G_{F}}{3} \tau \int_{x_{a}=\tau}^{x_{a}=1}\left[u^{a}\left(x_{a}\right) \bar{d}^{b}\left(\frac{\tau}{x_{a}}\right) \cos ^{2} \theta_{c}+u^{a}\left(x_{a}\right) \bar{s}^{b}\left(\frac{\tau}{x_{a}}\right) \sin ^{2} \theta_{c}+a \leftrightarrow b\right] \frac{d x_{a}}{x_{a}}$

Where $u^{a}$ is the $u$ quark structure function of hadron $a$ and $\bar{d}^{b}, \bar{s}^{b}$ are the down and strange anti-quark structure functions of hadron $b$. Since the Cabibbo angle is small, the following parton fusion processes are important for $\mathrm{W}$ production

$$
u \bar{d} \rightarrow W^{+} \text {and } \bar{u} d \rightarrow W^{-}
$$


In $\bar{p} p$ interactions, for large values of $\tau$, both the quark and the anti-quark may be valence. So near threshold, $\bar{p} p$ interaction cross sections for $W$ production will tend to be larger than $p p$ interactions. Another way of phrasing the same idea is that the annihilation cross section (the same as valence quark-antiquark fusion) will contribute at lower energies. As $s$ rises, $\tau \rightarrow 0$ and either or both of the fusing quarks may have to be obtained from the sea distributions. So $\bar{p} p$ and $p p \mathrm{~W}$ cross sections will tend toward each other as $s \rightarrow \infty$. Figure (46) shows the $W^{ \pm}$cross sections for a $W$ mass of $83 \mathrm{GeV} / \mathrm{c}^{2}[78]$. It immediately follows from the equation (66) that

$$
\frac{\sigma_{W} M_{W}^{3}}{\Gamma_{W \rightarrow u \bar{d}}}=G\left(\tau, Q^{2}\right)
$$

Where we have re-introduced the $Q^{2}$ dependence of the structure functions. If $Q^{2}$ is indeed fixed at $M_{W}^{2}$, then the above equation describes a general scaling law for $\mathrm{W}$ production. In fact this scaling law is found to be be valid for vector meson production in general, indicating that a common parton fusion mechanism may be the underlying process. Figure (47) shows the scaling law in operation for lower mass vector mesons. There should be a scale breaking tendency due to $Q^{2}$ dependences introduced by the different mass scales, but it is clearly a very weak one. It should be pointed out that the above scaling law can also be derived from dimensional arguments. The parton-fusion model however enables one to predict $G(\tau)$ from the structure functions. For $\mathrm{Z}$ production, analogous formulae hold. The fusing quarks are $u \bar{u}$ and $d \bar{d}$ respectively. The predictions of the masses and widths of the gauge bosons are given in table (2). Notice that the branching ratio $Z \rightarrow e^{+} e^{-}$is 0.031 whereas the branching ratio for $W \rightarrow e \nu$ is 0.083 . Allowing for the fact that there are two charge states for the $\mathrm{W}$, it is easier to observe the $\mathrm{W}$ at the collider than the $\mathrm{Z}$ by a factor of 5.4. There is a further suppression in the production cross section for $\mathrm{Z}$ since its mass is higher than the $\mathrm{W}$ and the total predicted suppression factor comes out to be closer to 10 at CERN energies.

\subsection{The discovery of the $W$ and the $Z$}

Figure (48) shows a schematic of the UA1 detector at CERN. The electromagnetic energy (photons, electrons and positrons) is captured in the elec- 


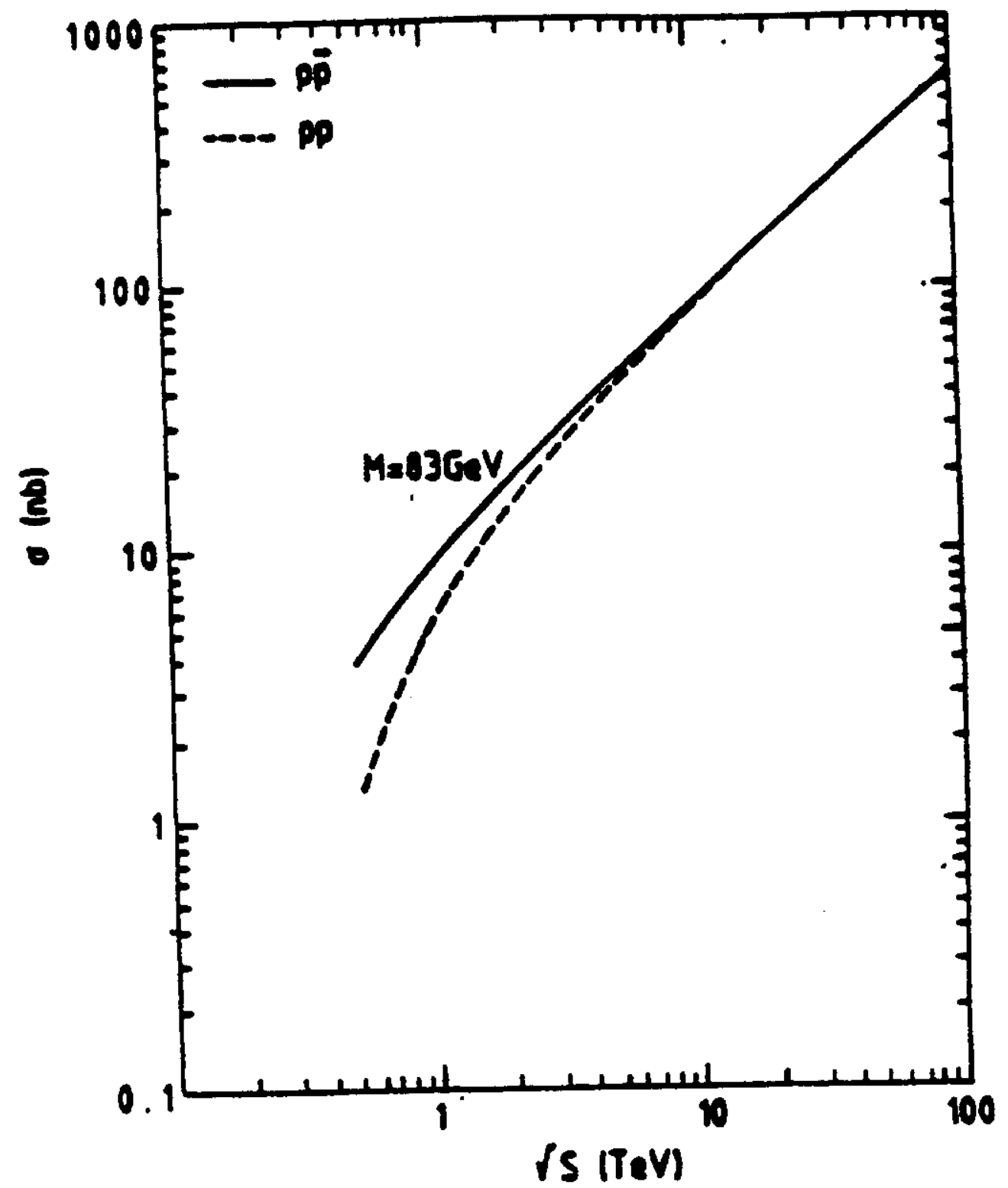

Figure 46: Theoretical prediction for $W^{ \pm}$production cross sections. The solid line is for $\bar{p} p$ collisions and the dashed line for $p p$ collisions. 


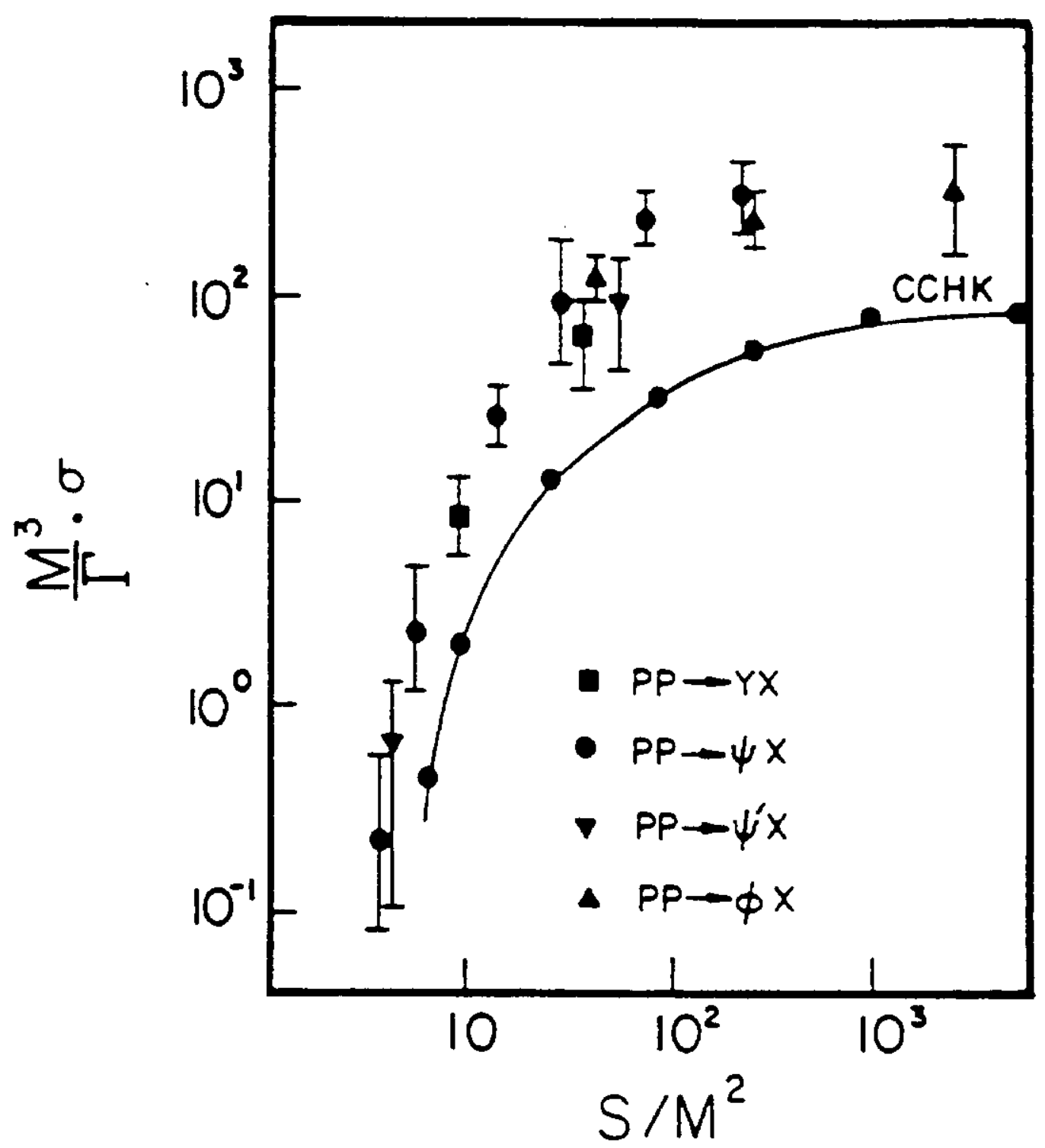

Figure 47: Scaling behavior of vector meson cross sections 


\begin{tabular}{|c|c|c|}
\hline Process & Standard Model Formulas & PredictedValue \\
\hline$W \rightarrow l \nu$ & $\left(G_{F} M_{W}^{3}\right) /(6 \pi \sqrt{2})$ & $250 \mathrm{MeV}$ \\
\hline$W \rightarrow$ hadrons & $3 D_{q} \Gamma_{W \rightarrow l \nu}$ & $2.25 \mathrm{GeV}$ \\
\hline$W \rightarrow$ all & $\left(3 D_{q}+D_{l}\right) \Gamma_{W \rightarrow l \nu}$ & \\
\hline 3 Generations & $12 \Gamma_{W \rightarrow l \nu}$ & $3.00 \mathrm{GeV}$ \\
\hline mass $_{\text {topquark }}>M_{W}$ & $9 \Gamma_{W \rightarrow l \nu}$ & $2.25 \mathrm{GeV}$ \\
\hline$Z \rightarrow \nu \bar{\nu}$ & $\left(G_{F} M_{Z}^{3}\right) /(12 \pi \sqrt{2})$ & $180 \mathrm{MeV}$ \\
\hline$Z \rightarrow e^{+} e^{-}$ & $\left.\left(1-4 x+8 x^{2}\right)\right) \Gamma_{Z \rightarrow \nu \bar{\nu}}$ & $91 \mathrm{MeV}$ \\
\hline$Z \rightarrow$ hadrons & $\left.3 D_{q}\left(2-4 x+40 x^{2} / 9\right)\right) \Gamma_{Z \rightarrow \nu \bar{\nu}}$ & $2.146 \mathrm{GeV}$ \\
\hline$Z \rightarrow$ all & $\left(G_{F} M_{Z}^{3} \sqrt{2}\right)\left(1-2 x+8 x^{2} / 3\right) D /(3 \pi)$ & $2.961 \mathrm{GeV}$ \\
\hline$\quad Z \rightarrow t \bar{t}$ & $\left.3\left(1-8 x / 3+32 x^{2} / 9\right)\right) \Gamma_{Z \rightarrow \nu \bar{\nu}}$ & $313 \mathrm{MeV}$ \\
\hline$M_{W}$ & $\sqrt{(\pi \alpha) /\left(G_{F} \sqrt{2} x\right)}$ & $78.2 \mathrm{GeV} / \mathrm{c}^{2}$ \\
\hline \multirow{2}{*}{\multicolumn{2}{|c|}{ after radiative corrections }} & (no corrections) \\
\hline & & $83.0 \pm 2.7 \mathrm{GeV} / \mathrm{c}^{2}$ \\
\hline \multicolumn{2}{|c|}{ after radiative corrections } & $\begin{array}{c}89.0 \mathrm{GeV} / \mathrm{c}^{2} \\
93.8 \pm 2.2 \mathrm{GeV} / \mathrm{c}^{2}\end{array}$ \\
\hline
\end{tabular}

$$
\begin{gathered}
x \equiv \sin ^{2} \theta_{W}=0.227 \pm .015 ;=0.217 \pm .062 \text { after corrections } \\
G_{F}=1.166 \times 10^{-5} \mathrm{Gev}^{-2} \\
D_{q}=\text { number of generations of quarks }=3 \\
D_{l}=\text { number of generations of leptons }=3 \\
D=\text { number of generations }=3
\end{gathered}
$$

Table 2: Standard model predictions for $\mathrm{W}, \mathrm{Z}$ masses and widths. For calculation of widths, the values of the boson masses after radiative correction have been used 
tromagnetic calorimeter known popularly as the 'gondolas'. Figure (49) illustrates how UA1 differentiates between electrons and muons. An electron is a charged track in the central drift chamber that points to a cluster of energy having the requisite electromagnetic shower shape in the gondolas. Cuts are introduced that require that the momentum measured in the magnetic field and the energy measured by the calorimeter match within errors. One also requires an absence of energy in the hadron calorimeter cells that are behind the electromagnetic cluster. In order to insure that the electron does not belong to a QCD jet, one demands that it is isolated, i.e. the accompanying energy in a cone surrounding the electron defined by $\Delta R \equiv \sqrt{\left(\Delta \eta^{2}+\Delta \phi^{2}\right)}<0.7$ (where $\eta$ is the pseudo-rapidity and $\phi$ is the azimuth in radians) be small. In order to be sure that the electron is the decay product of a massive particle, one demands that its transverse energy $E_{T}$ be greater than $15 \mathrm{GeV}$. The cuts used to arrive at the W and $\mathrm{Z}$ samples in the 1984 data by UA1 are illustrated in table (3).

Figure (50) is a typical W event on the interactive graphics facility where all tracks with transverse momentum greater than $1 \mathrm{GeV} / \mathrm{c}$ and all calorimeter cells with transverse energy greater than $1 \mathrm{GeV}$ are displayed. It can be seen that only the electron from the $W$ decay survives this cut. From the transverse energy imbalance of the event, the neutrino transverse vector may be deduced. The longitudinal component of the neutrino is hard to measure since there are tracks that escape down the beam pipe undetected.

Figure (51) is a typical $\mathrm{Z}$ event.[82][91]. There are two electromagnetic clusters with very little missing transverse energy. There is very little background activity once the $e^{+} e^{-}$pair have been removed from consideration.

Figure (52) shows the distribution of $\chi^{2} \equiv \operatorname{missing} E_{T}^{2} / \sigma^{2}$ missing $E_{T}$ for the $W$ events from UA1 for events from the $W$ selection procedure once the requirement that the missing $E_{T}$ is greater than $15 \mathrm{GeV}$. For low values of $\chi$, we see the characteristic gaussian shape of the average two jet event with very little missing $E_{T}$. This is followed by a tail of $\mathrm{W}$ events with large values of $\chi$. The curves are a prediction of a monte carlo with standard model parameters for the $W$ mass and width. The decay $W \rightarrow \tau \nu$ with $\tau \rightarrow e \nu_{r} \bar{\nu}_{e}$ contributes to the direct $W$ channel at lower values of missing 


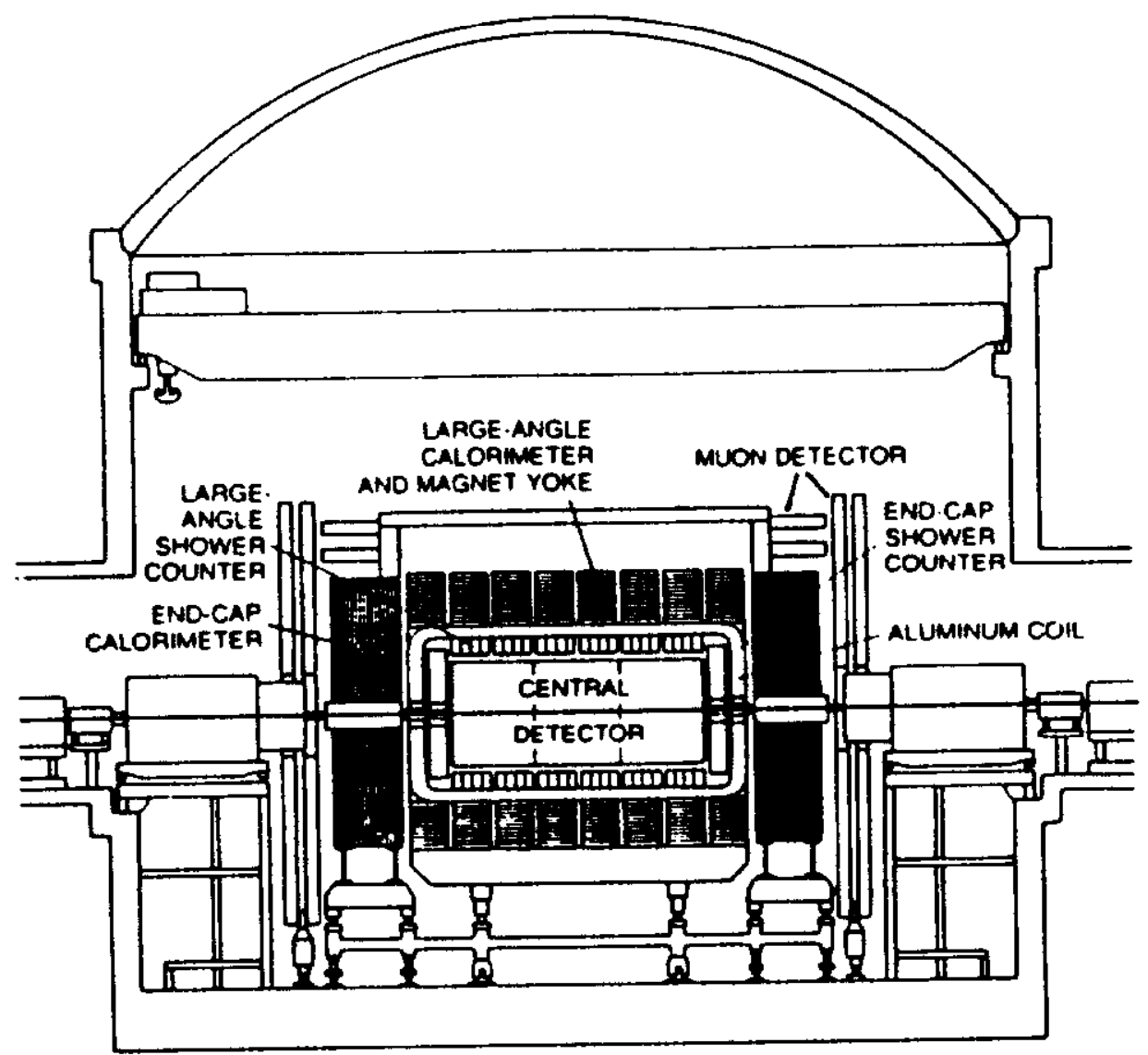

Figure 48: The UA1 detector at CERN 


\section{Electrons}
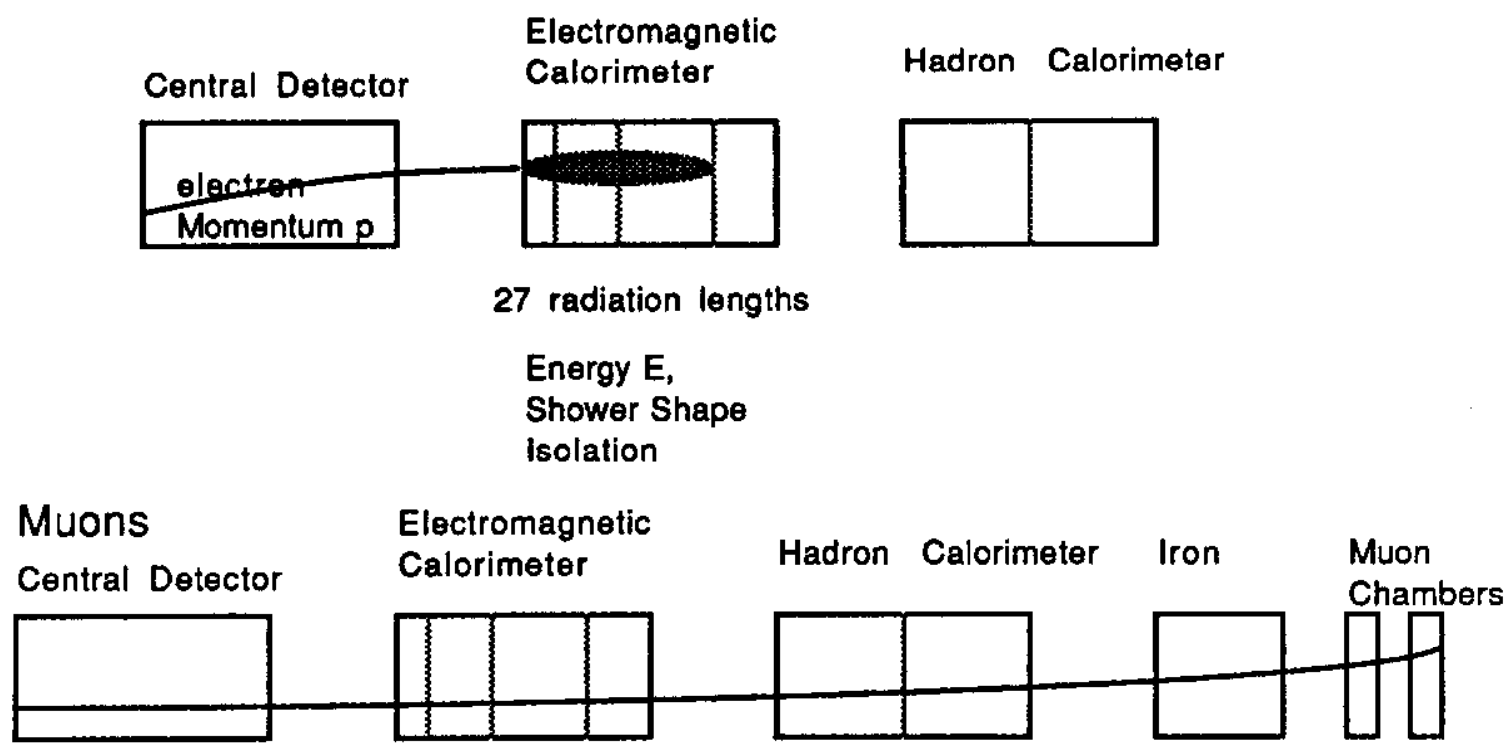

Minimum lonising Energy Loss

Figure 49: This figure illustrates how a typical calorimeter differentiates between electrons and muons

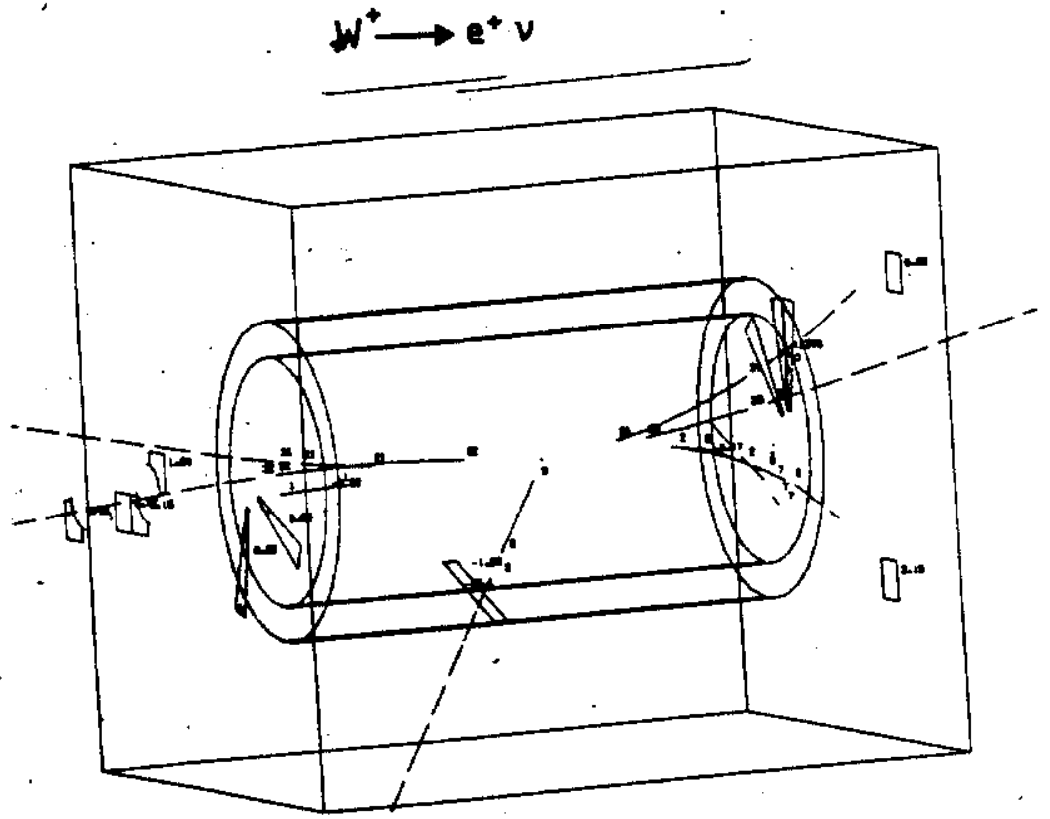

Figure 50: $A$ typical $W$ event 


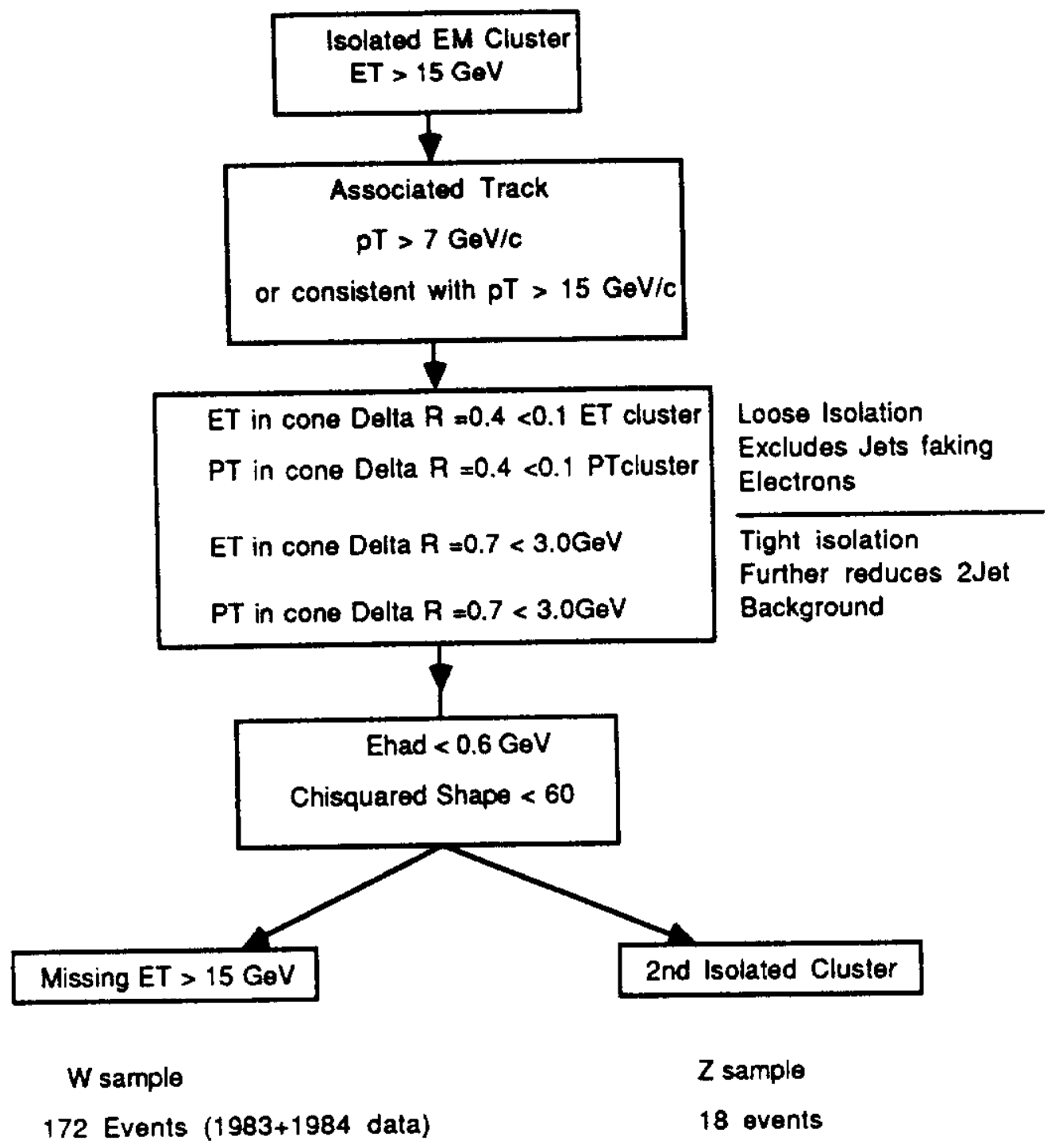

Table 3: Cuts used to arrive at the $\mathrm{W}$ sample for the 1984 UA1 data 

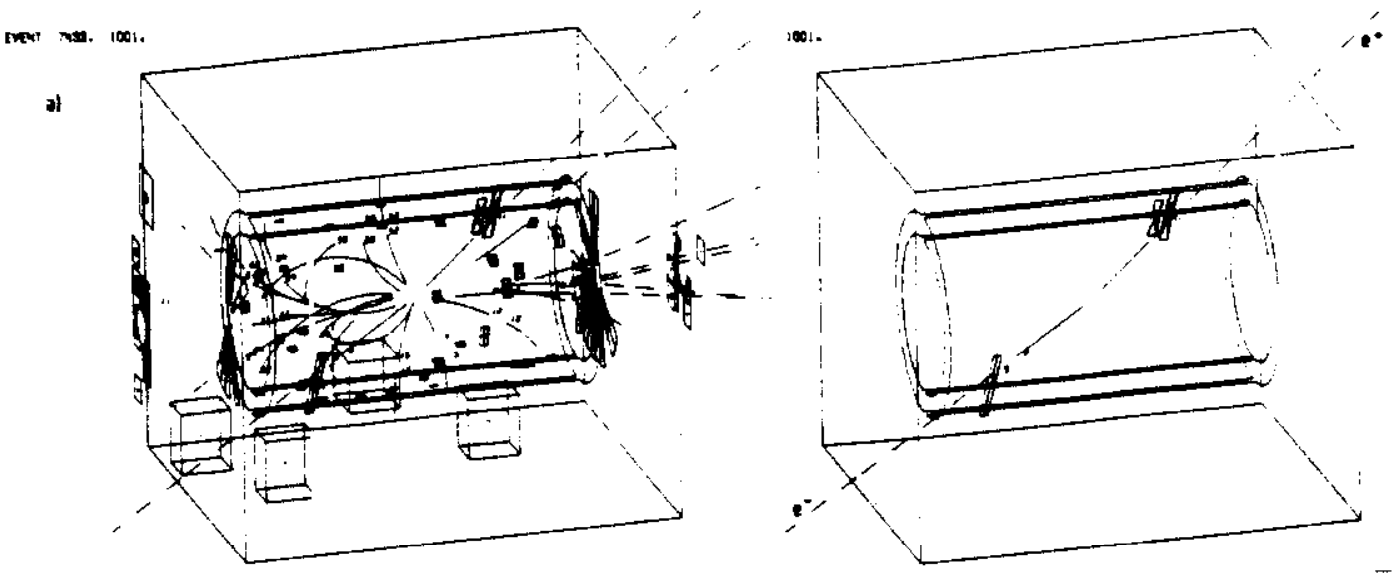

Figure 51: $A$ typical $Z$ event

$E_{T}$. This is because the neutrinos from the $r$ decay tend to reduce the missing $E_{T}$ due to the neutrino from the $\mathrm{W}$ decay.

Since the longitudinal component of the neutrino is not measured, we are reduced to considering only the transverse components of the $W$ decay products. The transverse mass of the $W, m_{T}$ is defined as

$$
m_{T}^{2}=2 E_{T}^{e} E_{T}^{\nu}\left(1-\cos \phi_{e v}^{T}\right)
$$

where $E_{T}^{a, v}$ are the transverse energies of the electron and neutrino respectively and $\phi_{c \nu}^{T}$ ) is the transverse opening angle between the electron and the neutrino. It is easy to show that the peak of the $m_{T}$ distribution occurs close to the mass of the $W$.

Figure(53) is the transverse mass distribution of the $W$ events from UA1. There is a sharp cut-off in the data at roughly the mass predicted by the standard model. This distribution is commonly known as the 'Jacobian peak'.

Figure (54) is the effective mass of the $e^{+} e^{-}$pairs from UA1. A clear peak at the expected $\mathrm{Z}$ mass value is seen with very little background.

$W$ decay has been observed to date into the channels $W \rightarrow e \nu, W \rightarrow \mu \nu$ 


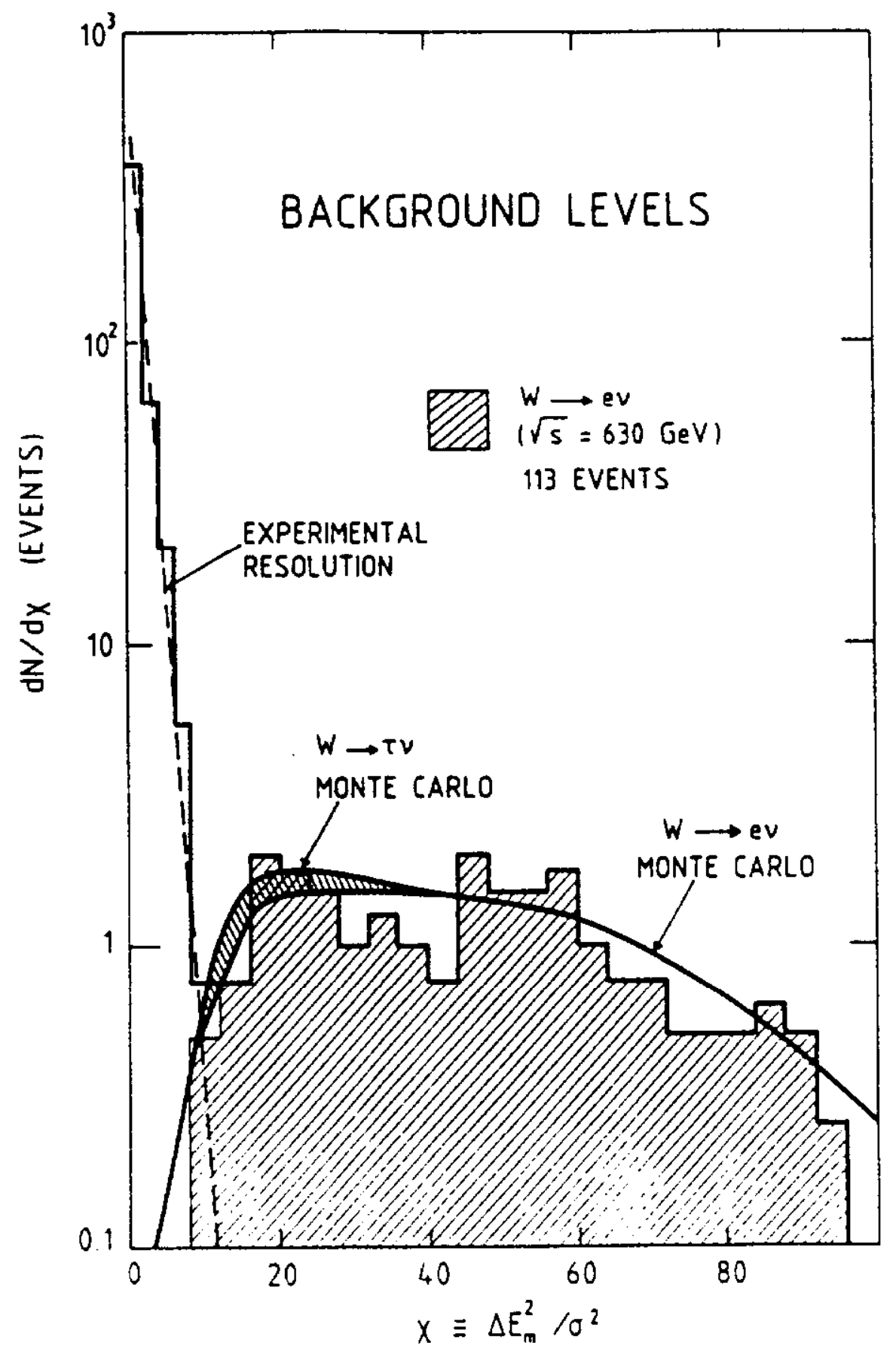

Figure 52: Background from two-jet fluctuations. This graph contains events from the $\mathrm{W}$ selection procedure after removing the requirement that the missing transverse energy is greater than $15 \mathrm{GeV}$. 


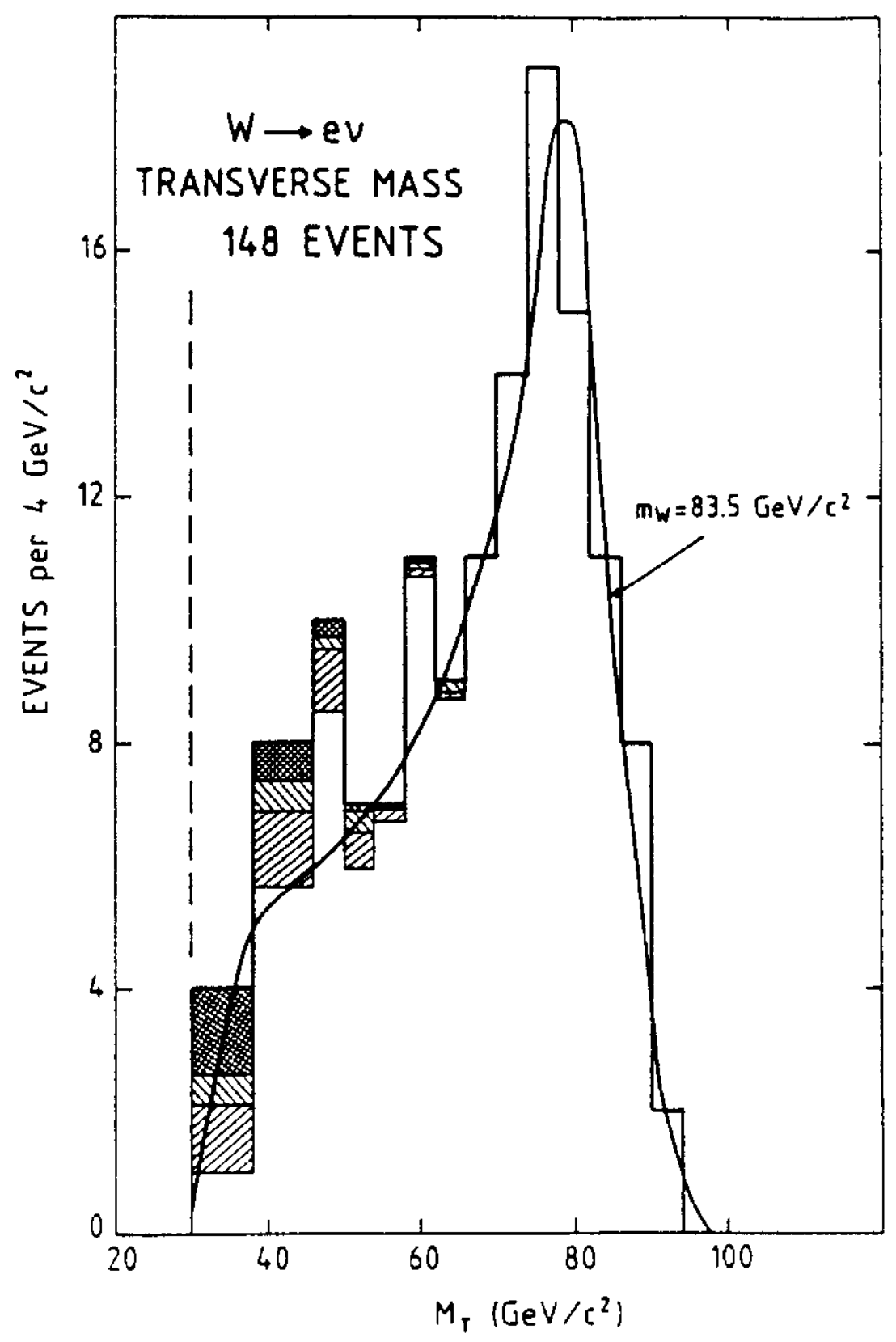

Figure 53: Transverse mass distribution of $\mathrm{W}$ events from UA1 


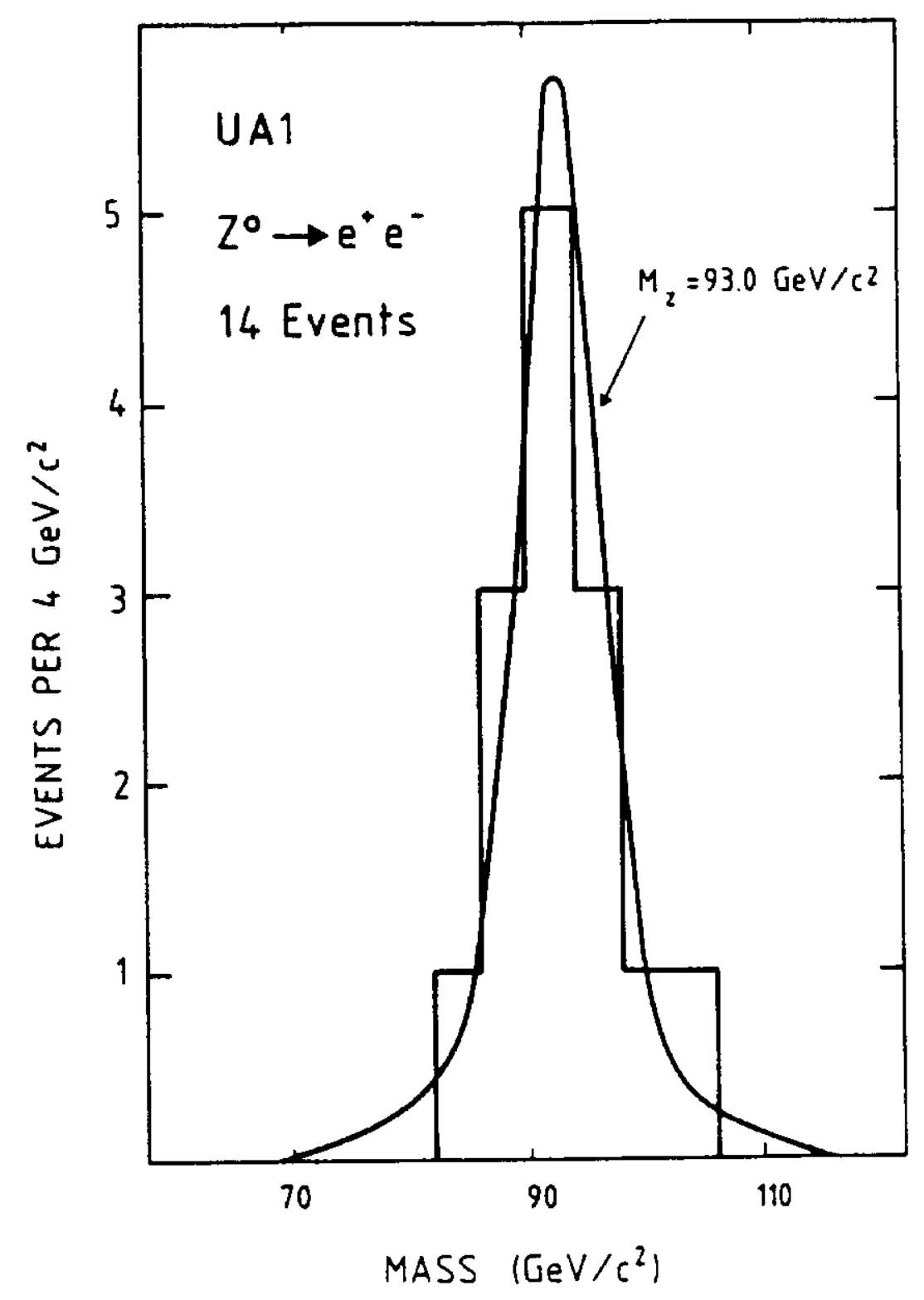

Figure 54: Effective mass distribution of electron pairs in the $\mathrm{Z}$ sample 


\begin{tabular}{|c|c|c|}
\hline & \multicolumn{2}{|c|}{$U A 1$} \\
\hline$\sqrt{s} G e V$ & 546 & 630 \\
\hline$\sigma_{W}^{\epsilon}(n b)$ & $0.55 \pm 0.08 \pm 0.09$ & $0.60 \pm 0.05 \pm 0.09$ \\
\hline$\sigma_{W}^{\mu}(n b)$ & $0.56 \pm 0.18 \pm 0.12$ & $0.67 \pm 0.08 \pm 0.14$ \\
\hline$\sigma_{W}^{\tau}(n b)$ & & $0.61 \pm 0.12 \pm 0.11$ \\
\hline$\sigma_{Z}^{e}(p b)$ & $42_{-18}^{+25} \pm 6$ & $73 \pm 14 \pm 11$ \\
\hline$\sigma_{z}^{\mu}(p b)$ & $100 \pm 50 \pm 15$ & $73 \pm 19 \pm 13$ \\
\hline & \multicolumn{2}{|c|}{$U A 2$} \\
\hline$\sigma_{W}^{e}(n b)$ & $0.57 \pm 0.1 \pm 0.07$ & $0.61 \pm 0.05 \pm 0.07$ \\
\hline$\sigma_{Z}^{e}(p b)$ & $112 \pm 37 \pm 9$ & $69 \pm 13 \pm 6$ \\
\hline & \multicolumn{2}{|c|}{ Theory } \\
\hline $\begin{array}{c}\sigma_{W}(n b) \\
\sigma_{Z}(p b)\end{array}$ & $\begin{array}{c}0.36_{-0.05}^{+0.11} \\
42_{-6}^{+13}\end{array}$ & $\begin{array}{c}0.45_{-0.08}^{+0.14} \\
51_{-10}^{+16}\end{array}$ \\
\hline
\end{tabular}

Table 4: $\mathrm{W}$ and $\mathrm{Z}$ production cross sections.branching ratios

and $W \rightarrow \tau \nu$ with the $\tau$ decaying into low multiplicity collimated jets ([81][94]). $\mathrm{Z}$ decay has been observed to date into $Z \rightarrow e^{+} e^{-}, Z \rightarrow \mu^{+} \mu^{-}$, and $z \rightarrow \tau^{+} \tau^{-}$with the $\tau^{s}$ decaying leptonically into $\mu^{+} \nu \bar{\nu}$ and $e^{-} \nu \bar{\nu}$.

Recently, UA2 have reported evidence for the decay [93] $W / Z \rightarrow q \bar{q}$ with the quark(anti-quark) fragmenting into jets. This process is very difficult to observe in practice because of the presence of a high degree of $Q C D$ background.

\subsection{W production properties}

\subsubsection{W cross section}

After correcting for acceptances and efficiencies, the $W$ cross section $\times$ branching ratio into $e \nu$ can be worked out. Table (3.4.1) contains the measurements of $\sigma . B$ at $\sqrt{s}$ of $546 \mathrm{GeV}$ and $630 \mathrm{GeV}$ by both UA1 and UA2. The theoretical predictions ([79]) are also shown and are in good agreement with the data. The predicted cross section increase from 546 $\mathrm{GeV}$ to $630 \mathrm{GeV}$ is in agreement with the data. The numbers for the $\mathrm{Z}$ 
cross section measurement are also given in the table (3.4.1).

\subsubsection{Longitudinal distribution of the $W$}

The longitudinal momentum of the $W$ is not directly measured since the neutrino longitudinal component is poorly measured. One can however impose the constraint that the mass of the $W$ is equal to the mass deduced from the transverse mass distributions. This results in two solutions for the neutrino 4 vector. In roughly one third of the cases, one of the solutions is unphysical. In another 30 percent, the ambiguity can be resolved after considering energy and momentum conservation for the whole event. For the remaining events, the solution with the smaller Feynman $x_{W}$ is chosen, since the overall predicted Feynman $x_{W}$ distribution is strongly peaked towards smaller values of $x_{W}$. Figure (55) is the Feynman $x_{W}$ distribution so obtained at $\sqrt{s}=546 \mathrm{GeV}$ and $630 \mathrm{GeV}$ respectively. The curves show predictions using the structure functions of Eichten et al [43] appropriately modified to take into account selection biases, experimental resolution and analysis bias due to the arbitrary resolution of the $x_{W}$ ambiguity. One can see that the shapes of the theoretical curves and the data are in agreement.

Using the relations $x_{a} x_{b}=\tau$ and $x_{a}-x_{b}=x_{W}$, one can determine the parton distributions in the proton and antiproton sampled in $\mathrm{W}$ production. Figure (56) shows the experimental values compared with the predicted values of Eichten et al.

With the charge of the $W$ determined, (there are events where the electron track is poorly measured so that its charge is ambiguous), it is possible to identify the proton (antiproton) parton with a $u(\bar{d})$ quark for $W^{+}$and a $d(\bar{u})$ quark for a $W^{-}$. the resulting $\mathrm{u}, \mathrm{d}$ quark distributions are shown in Figures (57) and (58). As can be seen the data and theory are in moderate agreement.

\subsubsection{W transverse distributions}

The $\mathrm{W}$ transverse momentum is obtained by summing up the transverse energy in the event vectorially for all particles except the lepton. Figure (59) shows the $p_{t}^{W}$ distribution compared with the theoretical predictions of Altarelli et al. The distribution peaks at $p_{t}^{W}$ of $4 \mathrm{GeV} / \mathrm{c}$. The hatched 


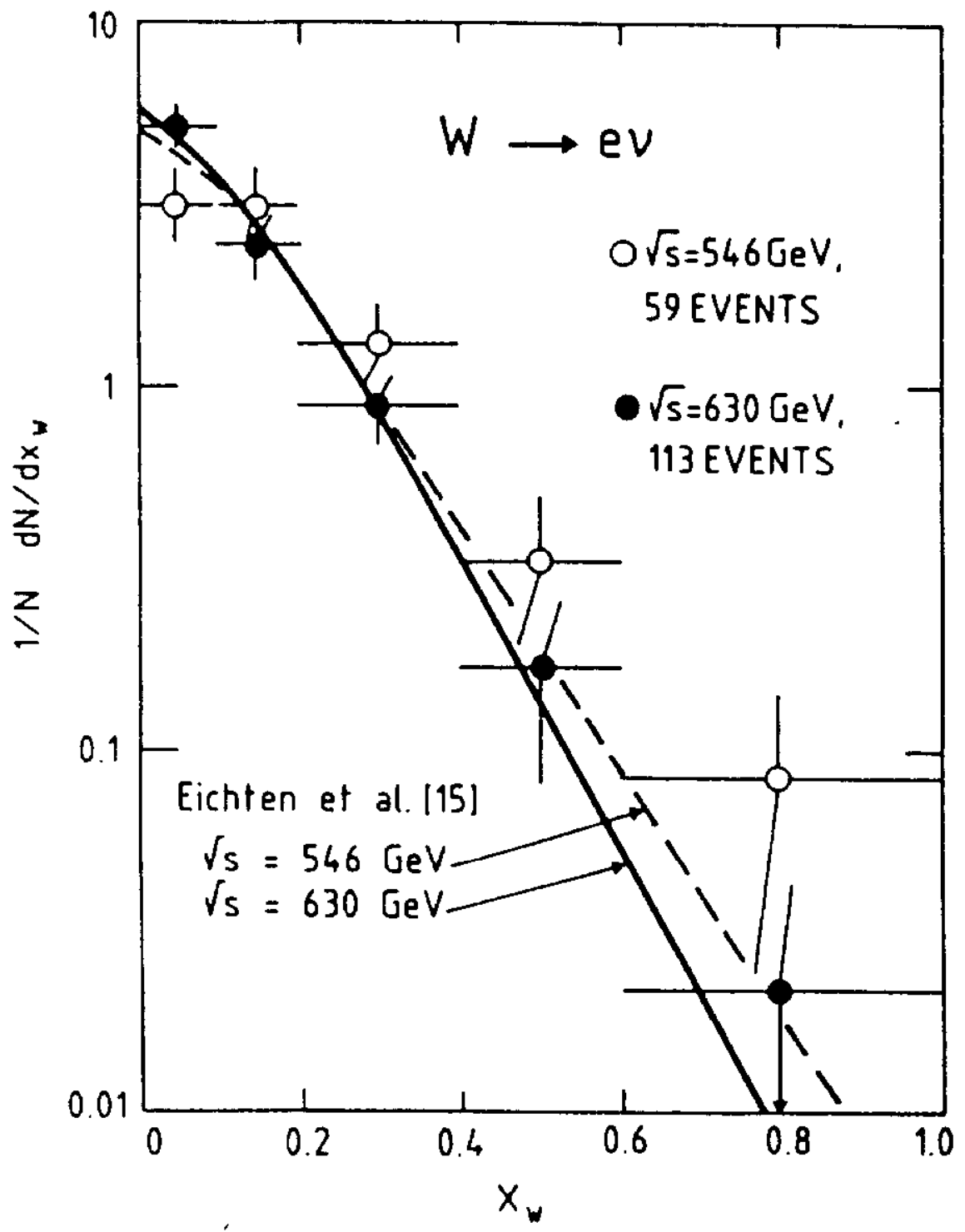

Figure 55: Feynman $x$ distribution of $W$ events from UA1. Open circles show $546 \mathrm{GeV}$ points and full circles $630 \mathrm{GeV}$ points respectively. The dashed curve is the prediction of Eichten et al for $540 \mathrm{GeV}$ and the full curve for $630 \mathrm{GeV}$. 


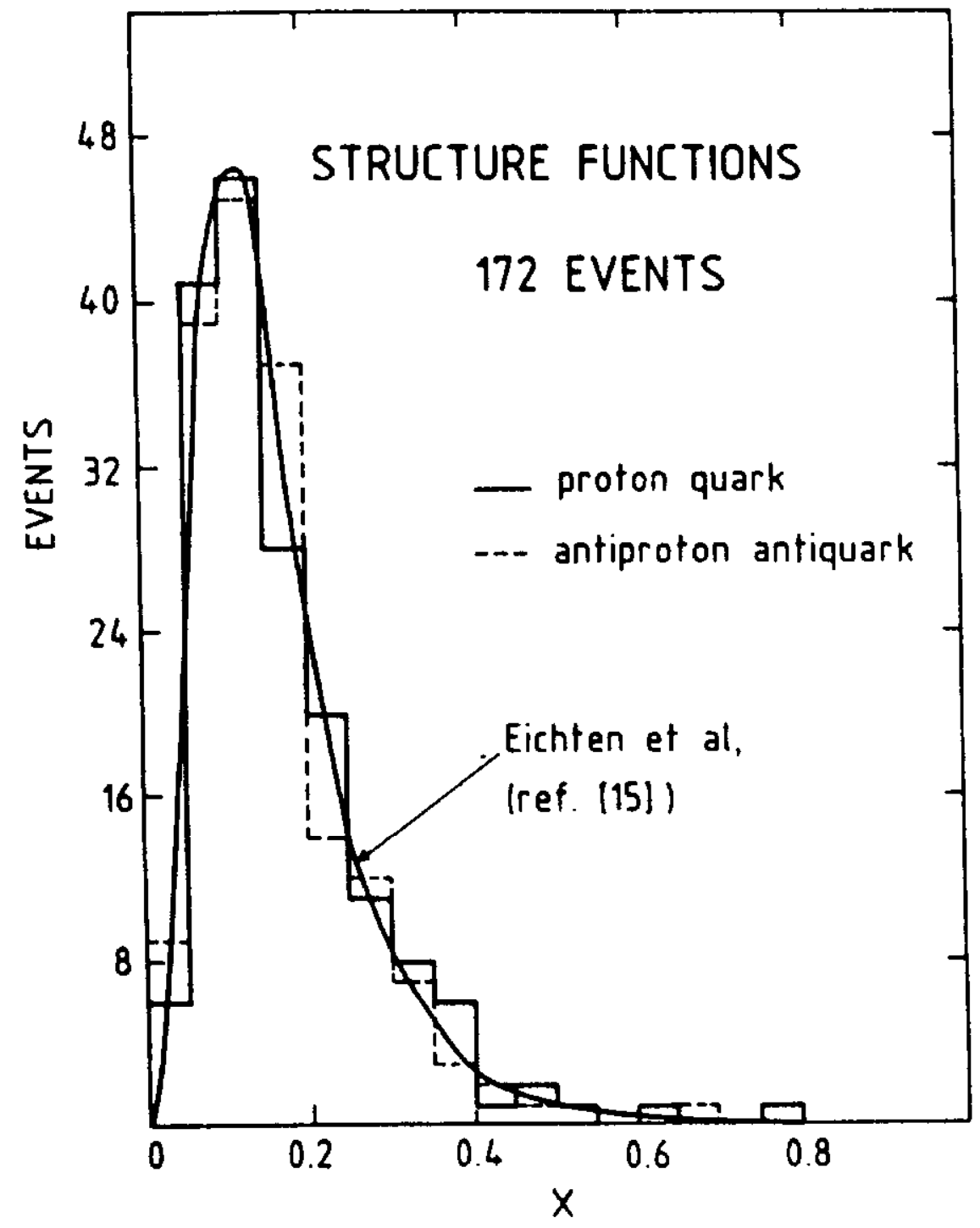

Figure 56: Experimental parton distributions in the proton(full histogram) and the antiproton(dashed histogram) 


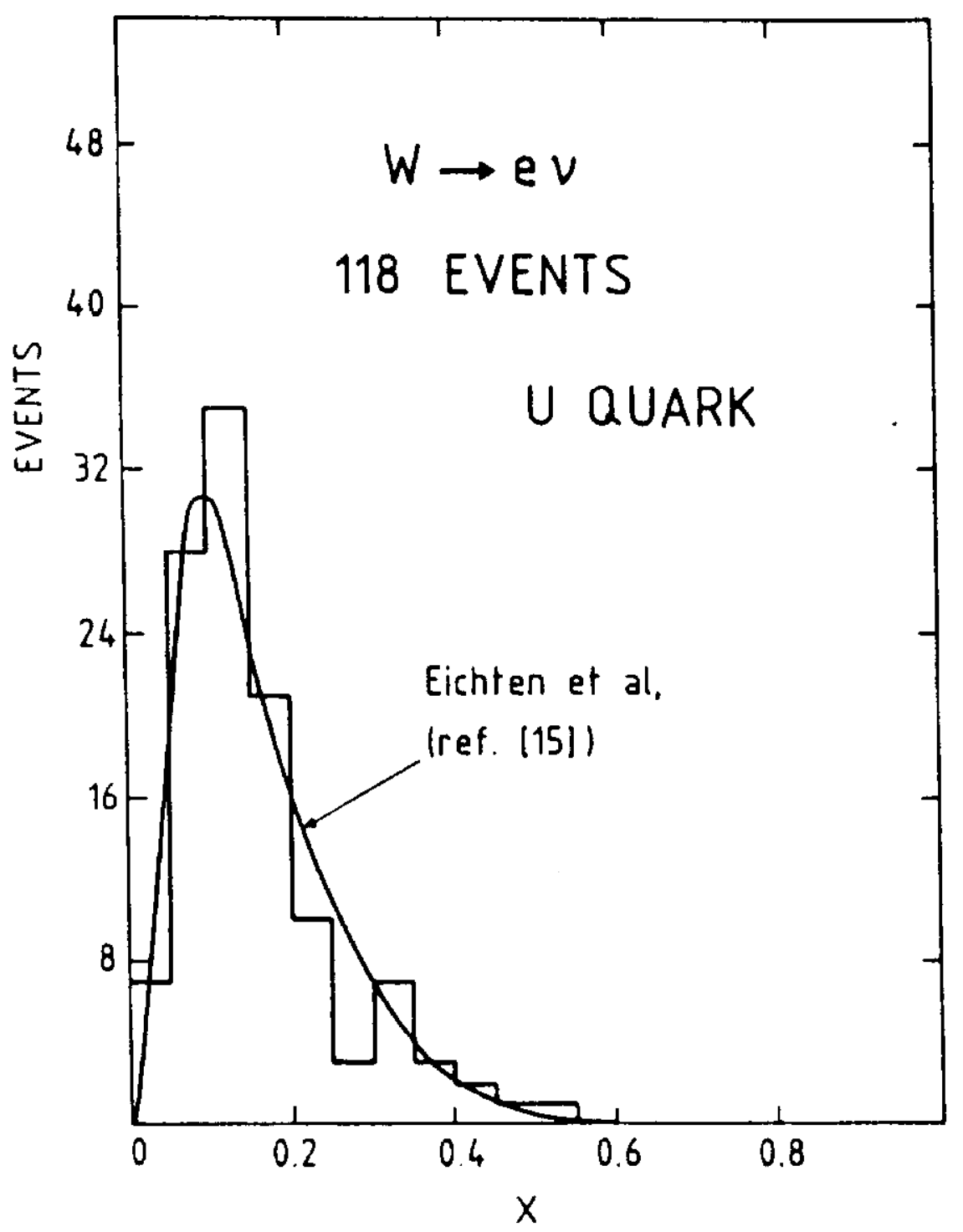

Figure 57: Up-quark distribution of the proton sampled in W production compared with the prediction of Eichten et al 


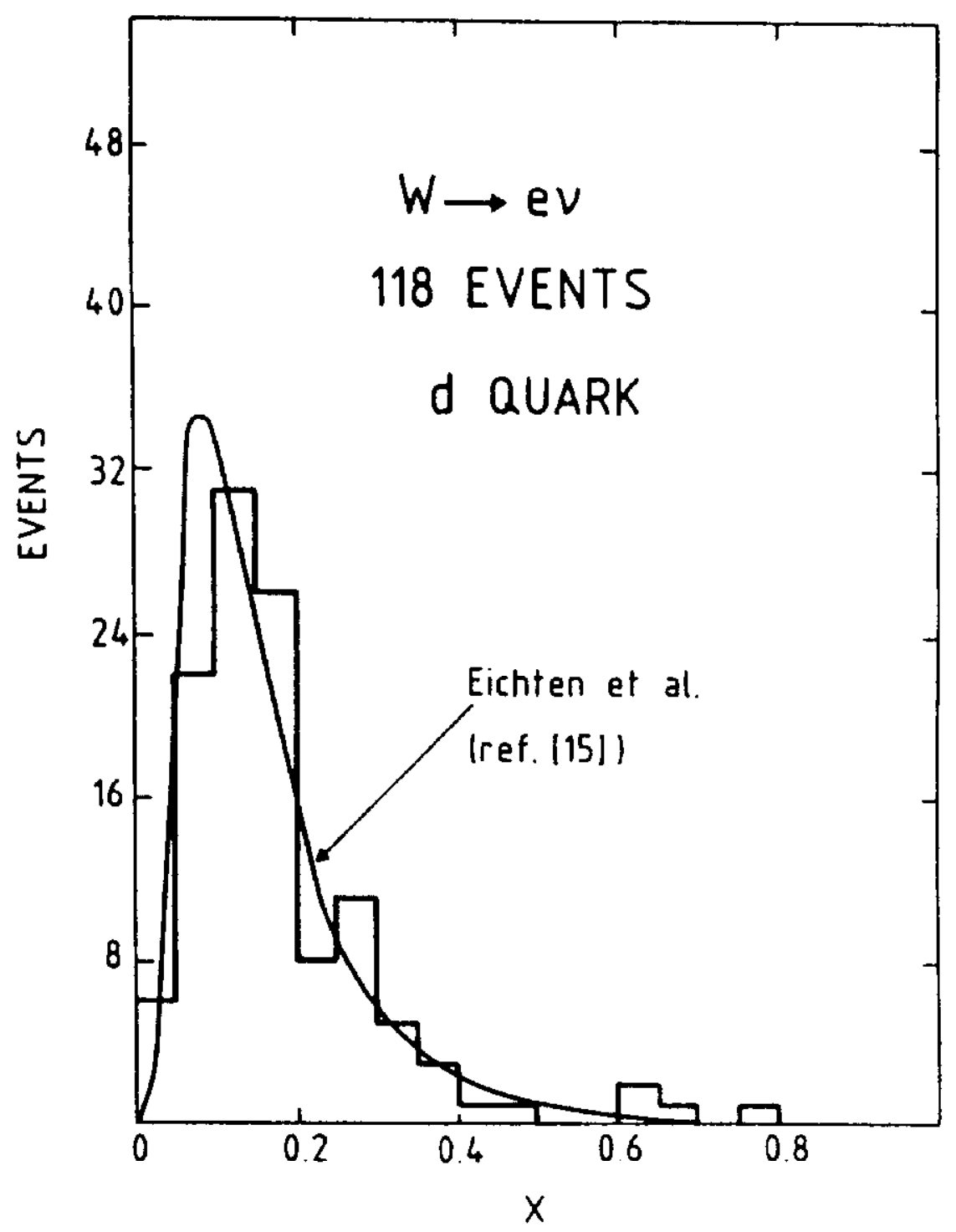

Figure 58: Down-quark distribution of the anti-proton sampled in W production compared with the prediction of Eichten et al 


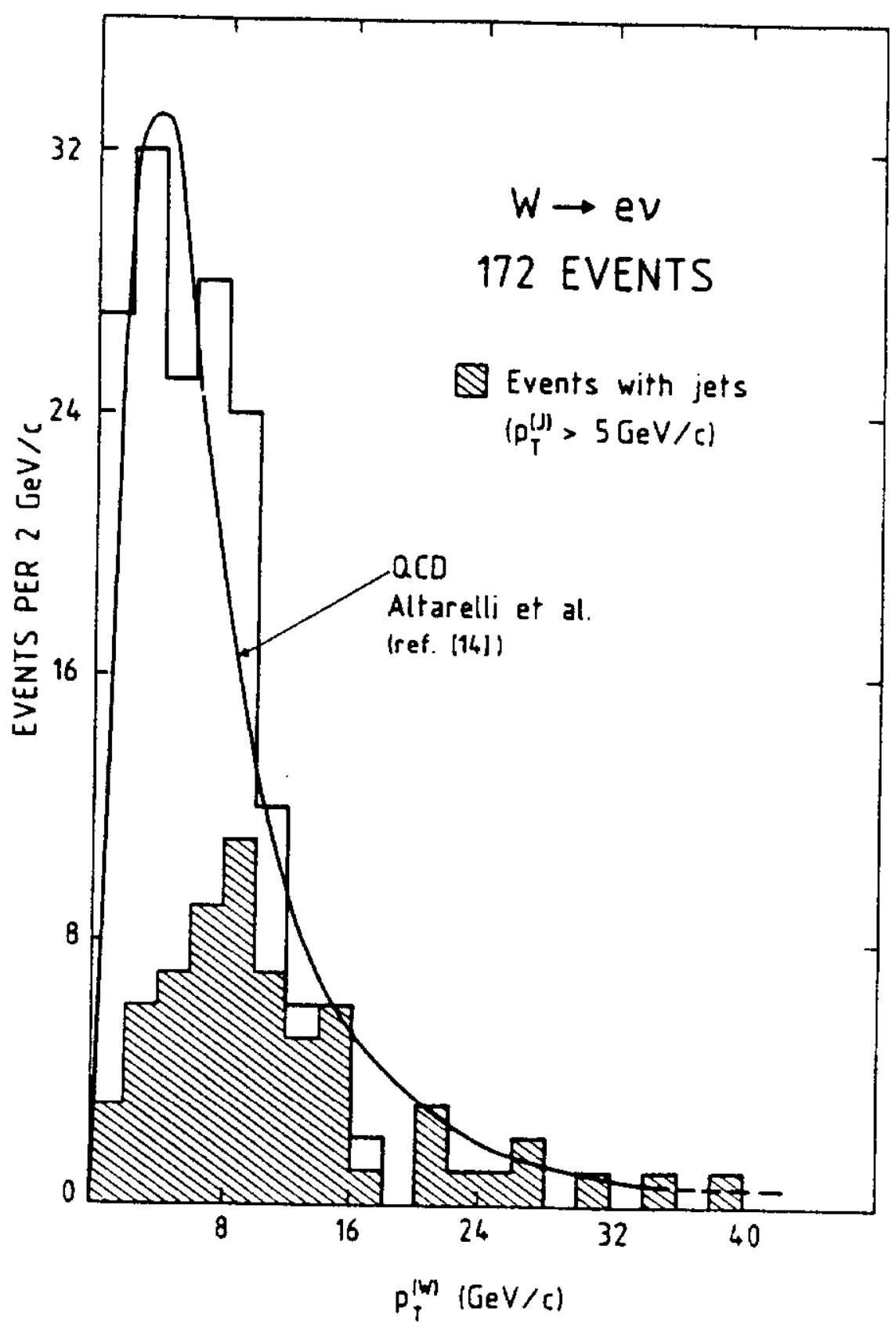

Figure 59: Transverse Momentum of the $\mathrm{W}$ compared with the prediction of Altarelli et al 
area of the histogram shows the contribution from events where the $W$ is recoiling against one or more hadronic jets. For high $p_{t}$ W's, it can be seen that nearly all events are jet-like. The angular distribution of the jets can be compared with QCD predictions ([80]). The distribution of $\cos \theta^{*}$, where $\theta^{*}$ is the angle between the jet and the average beam direction in the rest frame of the $W+$ jet(s) is strongly peaked towards the beam direction. This behavior is typical of initial state gluon bremsstrahlung. See Figure (60).

\subsection{W decay properties}

In order to measure the decay properties of the $\mathrm{W}$ boson, one needs to fit for the mass and width simultaneously. This is best done on the transverse mass distribution, using a maximum likelihood technique that takes into account measurement errors of individual events. Figure (53) shows such a fit to the transverse mass of the W using UA1 data. Using the measured value of the mass, one can, as explained in the previous section, solve for the neutrino longitudinal momentum and boost to the rest frame of the W. The angular distribution of the decay electron should exhibit maximal parity violation, if the standard V-A picture of parity violation is correct.

Figure (61) shows the mechanism of parity violation in $W^{+}$production at the $\bar{p} p$ collider. The fusing quark (u) should be left-handed. (negative helicity). This means that the $W^{+}$helicity should be +1 along the $\bar{p}$ direction. For the decay, the electron neutrino is left handed. The positron helicity points in the same direction as the neutrino helicity giving a total helicity component of +1 along the positron direction. The decay where the positron helicity is opposite to the neutrino helicity, giving a total helicity of zero, is suppressed by V-A. The angular distribution of the positron is then given by

$$
\frac{d N}{d \cos \theta}=\left|d_{11}^{1}(\cos \theta)\right|^{2}=(1+\cos \theta)^{2}
$$

i.e. the positrons prefer to go along the anti-proton direction. For the $W^{-}$ angular distribution, all arguments are reversed, with the electron tending to go along the proton direction.

Figure(62) shows the $\mathrm{W}$ decay angular distribution in the rest frame 


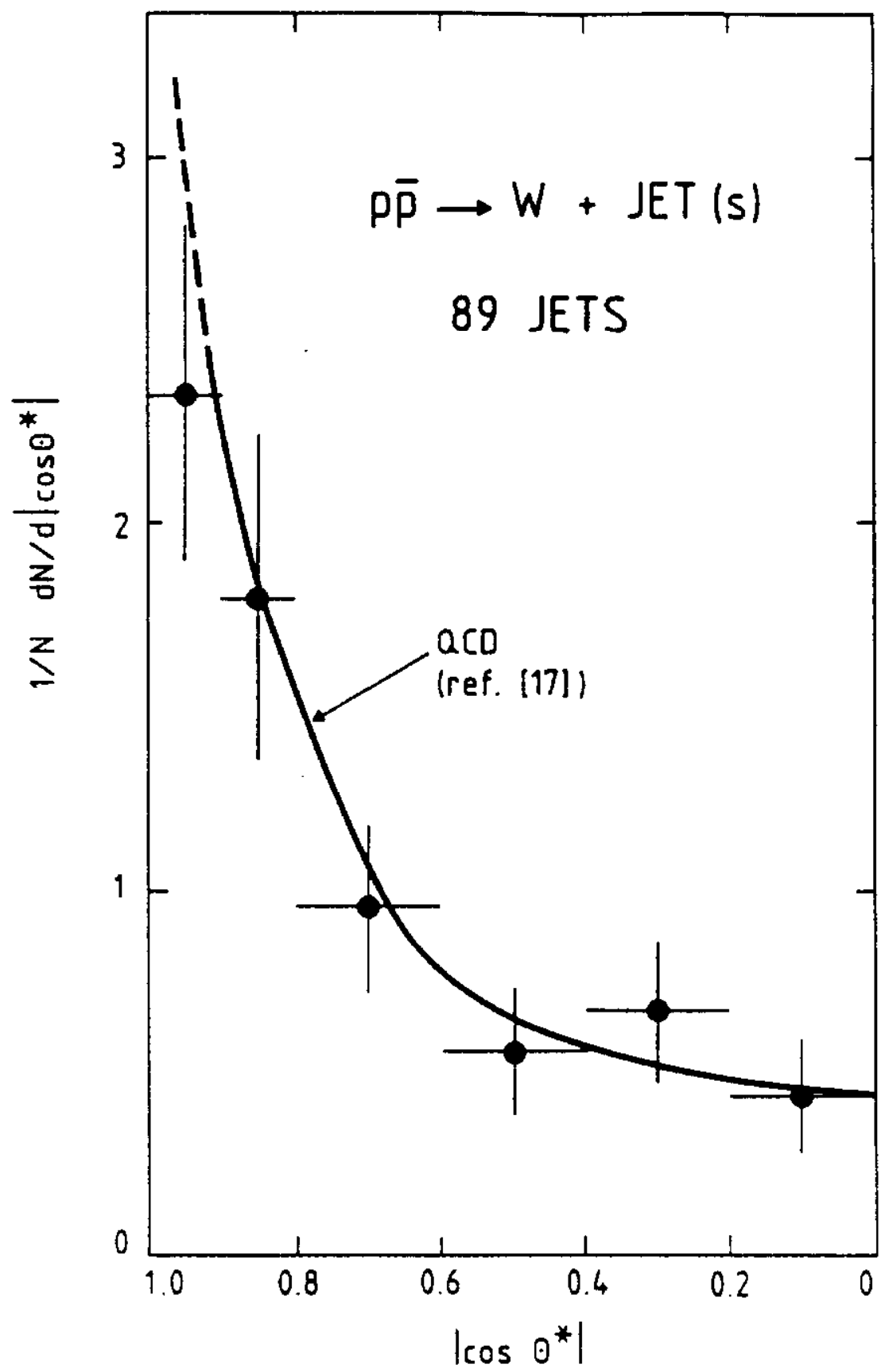

Figure 60: Angular distribution of the jet in the W-jet rest frame compared with the initial state bremsstrahlung QCD prediction. 


\section{Formation}

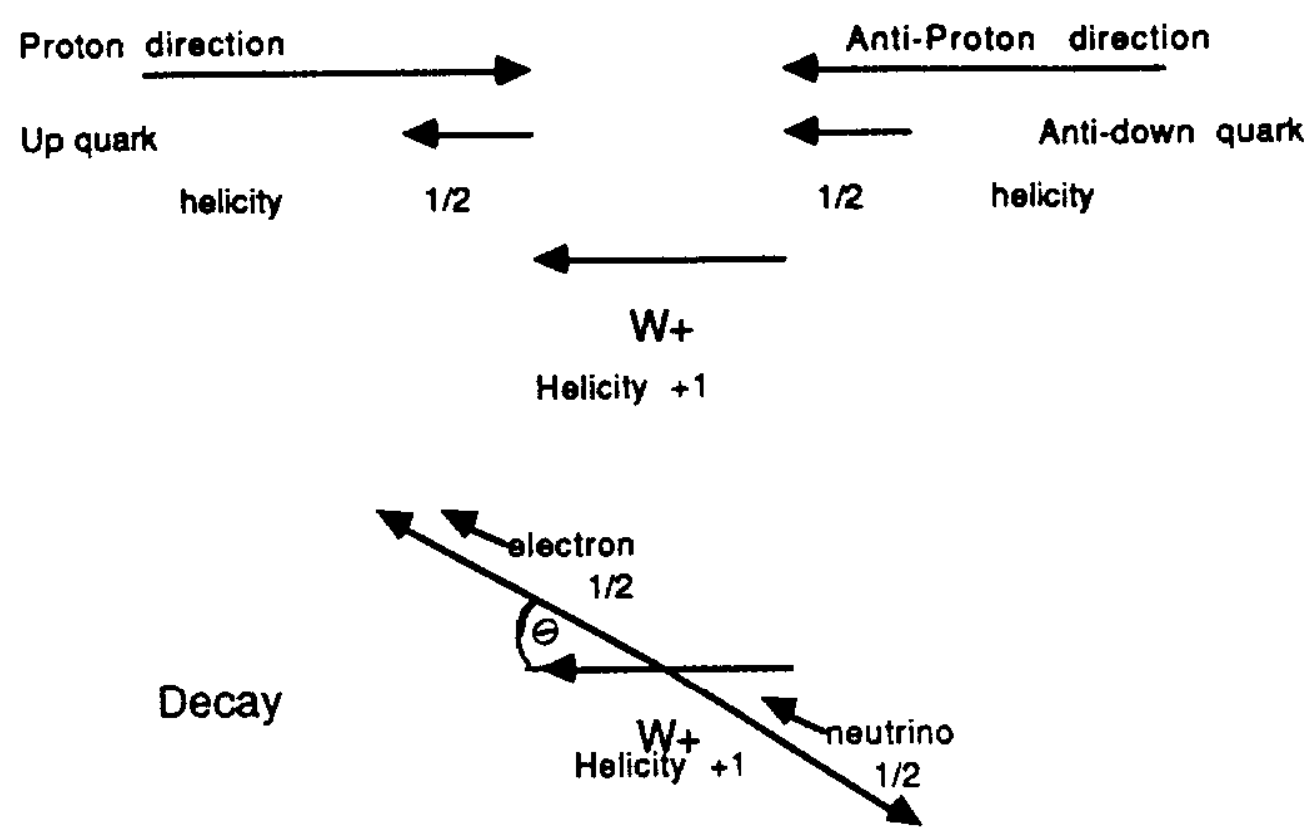

Figure 61: Mechanism of parity violation in $\mathrm{W}$ production and decay

of the $\mathrm{W}$ versus a curve for the $\mathrm{V}-\mathrm{A}$ expectation. Data are seen to be consistent with the theory.

\subsubsection{Standard Model parameters}

In the standard model, the masses of the gauge bosons may be expressed in terms of the Weinberg angle by the following equations

$$
\begin{gathered}
M_{W}^{2}=A^{2} /\left[(1-\Delta r) \sin ^{2} \theta_{W}\right] \\
M_{2}^{2}=A^{2} /\left[(1-\Delta r) \sin ^{2} \theta_{W} \cos ^{2} \theta_{W}\right]
\end{gathered}
$$

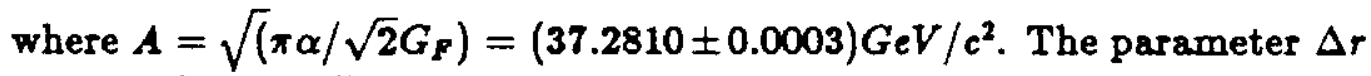
accounts for the effect of one-loop corrections to the $W$ and $Z$ masses and has been calculated to be $0.0711 \pm 0.0013[95]$. Using equations (71) and (72), we can determine $\sin ^{2} \theta_{W}$ two different ways. The first is to use the ratio $M_{W} / M_{Z}$ which is free of calibration systematics to first order. This results in the quoted values of $\sin ^{2} \theta_{W}^{a}$ in table (6). The second method is to use the calculated value of $\Delta r$ and simultaneously fit equations (71) and (72). This 


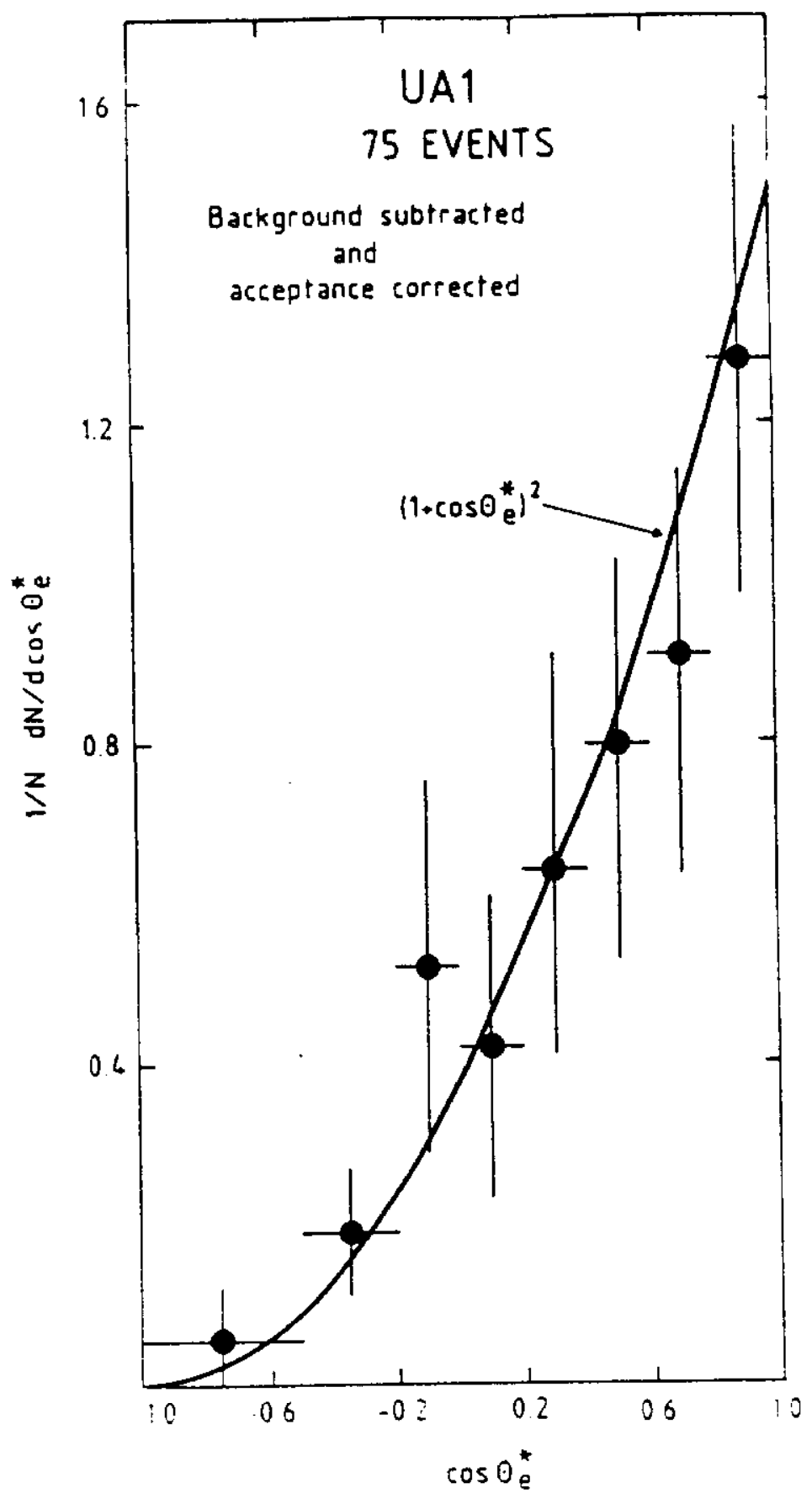

Figure 62: Angular distribution of the decay electron(positron) in the $W$ rest frame compared with the prediction of V-A theory 
gives the values quoted in table (6) as $\sin ^{2} \theta_{W}^{b}$. The $\rho$ parameter can also be estimated from the relation $\rho=M_{W}^{2} / M_{Z}^{2} \cos ^{2} \theta_{W}$. In the minimal standard model $\rho=1$. Equations (71) and (72) can also be used to determine $\Delta r$. This value is quoted as $\Delta r^{a}$. Using the value of $\sin ^{2} \theta_{W}$ from low energy experiments [97] $(=0.232 \pm 0.004($ exp $) \pm 0.003$ (theor) $)$, one can determine another value of $\Delta r$. This is quoted as $\Delta r^{b}$ in table (6). The masses of the gauge bosons obtained by fitting to the data are given in table (5). Both UA1 and UA2 values are quoted. UA1 has used the muon and tau decay channels as well to obtain mass values, though with increased errors. It can be seen that all the measurements are consistent with each other and with the standard model. [96]

\subsection{Number of neutrino species}

The measured rates of production of the W, $\mathrm{Z}$ system can be used to set upper limits on the number of light neutrino species in the universe [98]. The basic assumptions are

- The neutrino is light so that $\Gamma(Z \rightarrow \nu \bar{\nu})$ is the same as that for the known species.

- The associated lepton is heavier than the W so that the total width of the $W$ is unaffected.

Under these assumptions, additional light neutrinos will cause an increase in the $\mathrm{Z}$ width without the $\mathrm{W}$ being affected. This will cause a decrease in the branching ratio $Z \rightarrow e^{+} e^{-}$with the consequence that $\mathrm{Z}$ decays will increasingly be difficult to observe. The ratio

$$
\begin{gathered}
R=\frac{\sigma \cdot B(W)}{\sigma . B(Z)}=\frac{\Gamma(W \rightarrow e \nu)}{\Gamma\left(Z \rightarrow e^{+} e^{-}\right)} \frac{\Gamma_{Z}}{\Gamma_{W}} \frac{\sigma_{W}}{\sigma_{Z}} \\
R=2.715 \frac{\Gamma_{Z}(\text { standard })+\left(N_{\nu}-3\right) \Gamma_{Z \rightarrow \nu \bar{\nu}}}{\Gamma_{W}(\text { standard })}(3.3 \pm 0.2)
\end{gathered}
$$

where the ratio $\Gamma_{W \rightarrow e \nu} / \Gamma_{Z \rightarrow e e}$ is obtained from the standard model couplings to be 2.715 and the ratio $\sigma_{W} / \sigma_{Z}$ is predicted by the fusion model to be $3.3 \pm 0.2$. The error in the latter ratio is due to the error in determining 


\begin{tabular}{|c|c|}
\hline Parameter & UA1 \\
\hline electron decay & 1985 data excluded \\
$M_{W}$ & $83.5_{-1.1}^{+1.0}$ stat \pm 2.7 syst. \\
$\Gamma_{W}$ & $<6.5(90 \% C L)$ \\
$M_{Z}$ & $93.0 \pm 1.4$ stat. \pm 3.0 syst. \\
$\Gamma_{Z}$ & $<8.3(90 \% C L)$ \\
\hline Muon decay & $80.7_{-4.1}^{+4.5}$ stat..$_{-8.1}^{+9.0}$ syst. \\
$M_{W}$ & $96.8_{-3.1}^{+3.3}$ stat..$_{-4.0}^{+4.3}$ syst. \\
$M_{Z}$ & \\
\hline Tau decay & $89 \pm 3$ stat. \pm 6 syst. \\
$M_{W}$ & UA2 \\
\hline Parameter & $80.1 \pm 0.6$ stat. \pm 0.5 syst. \pm 1.3 syst. \\
\hline$M_{W}$ & $<7.0(90 \% C L)$ \\
$\Gamma_{W}$ & $<2.1 \pm 1.1$ stat. \pm 1.5 syst. \\
$M_{Z}$ & $<5.8(90 \% C L)$ \\
$\Gamma_{Z}$ & \\
\hline
\end{tabular}

Table 5: Summary of measurements of the $\mathrm{W}$ and $\mathrm{Z}$ mass and width in $\mathrm{UA} 1$ and UA2. Units $\mathrm{GeV} / \mathrm{c}^{2}$. 


\begin{tabular}{|c|c|c|}
\hline Parameter & \multicolumn{2}{|c|}{$U A 1$} \\
\hline & Electron & Muon \\
\hline $\sin ^{2} \theta_{W}^{a}$ & $0.194 \pm 0.031$ & $0.31 \pm 0.18$ \\
$\sin ^{2} \theta_{W}^{b}$ & $0.214_{-0.006}^{+0.005} \pm 0.015$ & $0.228_{-0.057}^{+0.051}$ \\
$\rho$ & $1.026 \pm 0.037 \pm 0.019$ & \\
\hline Parameter & $U A 2$ & \\
\hline $\sin ^{2} \theta_{W}^{a}$ & $0.242 \pm 0.023 \pm 0.009$ & \\
$\sin ^{2} \theta_{W}^{b}$ & $0.232 \pm 0.004 \pm 0.008$ & \\
$\rho$ & $0.988 \pm 0.027 \pm 0.006$ & \\
$\Delta r^{a}$ & $0.105 \pm 0.077 \pm 0.029$ & \\
$\Delta r^{b}$ & $0.069 \pm 0.026 \pm 0.030$ & \\
\hline
\end{tabular}

Table 6: Measurements of the Standard Model parameters in UA1 and UA2

the structure functions. As the statistics of the experiments improve, this theoretical error will prove to be the ultimate limitation of this method.

The mass of the top quark adds yet another level of uncertainty to this calculation. If the top exists at a mass less than $M_{Z} / 2$, then $Z \rightarrow t \bar{t}$ and $W \rightarrow t \bar{b}$ will exist. If $M_{Z} / 2<M_{t}<M_{W}$, then only the latter decay is allowed. If $M_{t}>M_{W}$, then the top quark does not enter into this problem. The measured value of $\mathrm{R}$ for UA1 is $8.9+1.6-1.3$. This leads to a limit on the number of neutrino species at $90 \%$ confidence level as a function of the top quark mass as shown in figure (63).

\subsection{Higher mass W's and Z's and other exotic phe- nomena}

Since no $W \rightarrow e \nu$ candidates have been observed with transverse mass in excess of the expected distribution of the $W$ decays and no $Z \rightarrow e^{+} e^{-}$ candidates have been observed far in excess of the $Z$ mass, it is possible to set limits on the masses of the higher lying W's and Z's provided one assumes that they are coupled to the quarks in the same way. UA1 quotes 


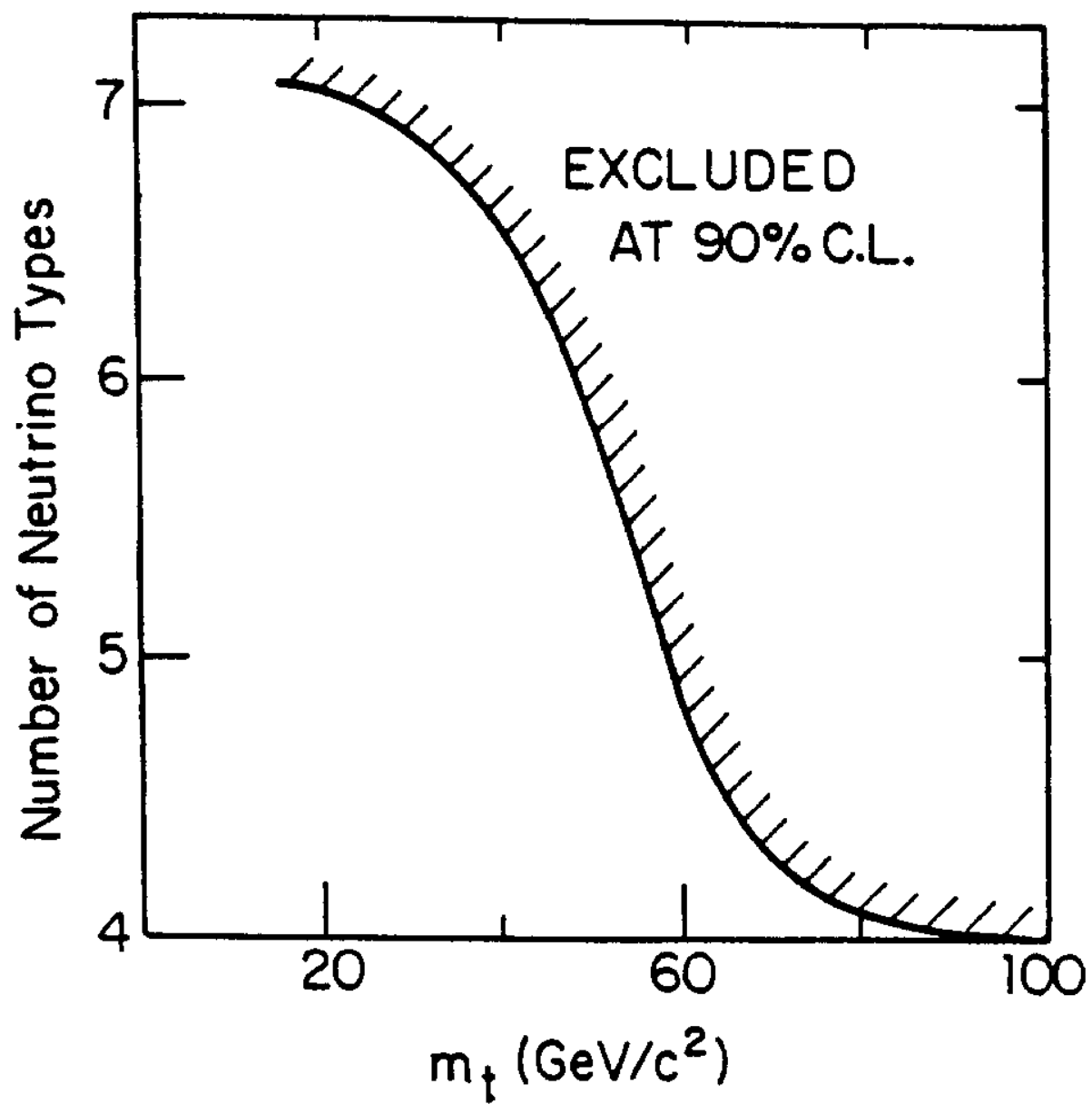

Figure 63: Upper limit to the number of neutrino species in the universe as a function of the top quark mass 
limits of [99] $M_{W}^{\prime}>220 \mathrm{GeV} / c^{2}$ and $M_{Z}^{\prime}>166 \mathrm{GeV} / c^{2}$ at $90 \%$ confidence level. UA1, however, does report two very high $p_{t}$ W's [100] which are in excess of the expected QCD background ( 2 observed where 0.1 event is expected). At present, these events offer perhaps the only hint at energies up to the CERN collider that the Standard model has limitations.

To conclude, $\mathrm{W}$ and $\mathrm{Z}$ production and decay properties conform very well to the expectations of the Salam-Weinberg model of electro-weak interactions. References ([81]-[89]) and ([90]-[94]) offer a semi-exhaustive list of publications on the subject from UA1 and UA2 collaborations respectively. The production cross sections are higher at the Tevatron than $s \bar{p} p s$ by a roughly factor of three, and the higher statistics this will provide at Fermilab will lead to tighter limits on non-standard model processes than exist at present. A precise determination of the $W / Z$ mass difference becomes possible, once the $\mathrm{Z}$ mass is well measured at the SLC. The $\mathrm{W} / \mathrm{Z}$ mass difference can then be determined by the Fermilab colliding detector experiments, CDF and D0 to a precision that will have further bearing on the Higgs and top quark masses.

\section{Heavy Quark Production}

One of the outstanding questions still to be answered within the standard model is "does the top quark exist and if so what is its mass?". The UAl collaboration has reported preliminary evidence for the top quark in their 1983 data ([101]) but has since then not followed this up with confirmatory evidence. In this chapter we will examine

- The mechanisms for heavy flavor production

- $b \bar{b}$ production and reported evidence for $b \bar{b}$ mixing.

- Evidence for top quark production

- Potential of the Tevatron for finding the top quark 


\subsection{Production Mechanisms}

There exist basically three mechanisms for the production of heavy quark Q.

- Flavor creation $g g \rightarrow Q \bar{Q}, q \bar{q} \rightarrow Q \bar{Q}$

- Flavor excitation $g Q \rightarrow g Q ; q Q \rightarrow q Q$, where there is an intrinsic $Q$ component associated with the structure function in the hadron (see figure (32)).

- Gluon splitting which is a higher order process with $g g \rightarrow g g$; one of the final state gluons splitting into $g \rightarrow Q \bar{Q}$. This process contributes due to the overwhelming predominance of the $g g \rightarrow g g$ process.

\subsubsection{Flavor creation}

Figure (64) gives the lowest order Feynman graphs for the production of the $Q \bar{Q}$ final state. The process (a) is a coherent sum of the three diagrams where the $Q \bar{Q}$ state is produced by $g g$ collisions. The first of these has a gluon exchange in the $\hat{s}$ channel and the other two have the heavy quark exchange in the $\hat{t}$ and the $\hat{u}$ respectively. The second process (b) is due to the $q \bar{q}$ initial state. One can write, with $m$ the heavy quark mass,

$$
\frac{d \hat{\sigma}}{d \hat{t}}(g g \rightarrow Q \bar{Q})=\frac{\pi \alpha_{\theta}^{2}}{\hat{s}^{2}}|A|^{2}
$$

where

$$
\begin{gathered}
|A|^{2}=\frac{1}{8}\left(\frac{6}{\hat{s}^{2}}\left(\hat{t}-m^{2}\right)\left(\hat{u}-m^{2}\right)\right. \\
+\left[\frac{4\left(\hat{Q}-m^{2}\right)}{3\left(\hat{t}-m^{2}\right)}-\frac{\left.8 m^{2}(\hat{\imath})+m^{2}\right)}{3\left(\hat{t}-m^{2}\right)^{2}}+\frac{3\left(\hat{t}-m^{2}\right)\left(\hat{\imath}-m^{2}\right)+m^{2}(\hat{Q}-\hat{t})}{3\left(\hat{t}-m^{2}\right)}\right. \\
\left.+\hat{t} \leftrightarrow \hat{u}]-\frac{m^{2}\left(\hat{3}-4 m^{2}\right)}{3\left(\hat{t}-m^{2}\right)\left(\hat{\imath}-m^{2}\right)}\right)
\end{gathered}
$$

The first term with $\hat{s}^{2}$ in the denominator is due to $\hat{s}$ channel gluon exchange. The second,third and fourth with $\left(\hat{t}-m^{2}\right)$ in the denominator is due to heavy quark exchange in the $\hat{t}$ channel and the $\hat{u}$ channel exchange 

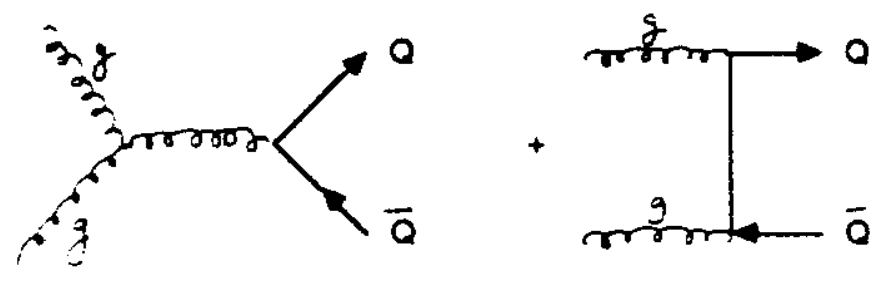

(a)
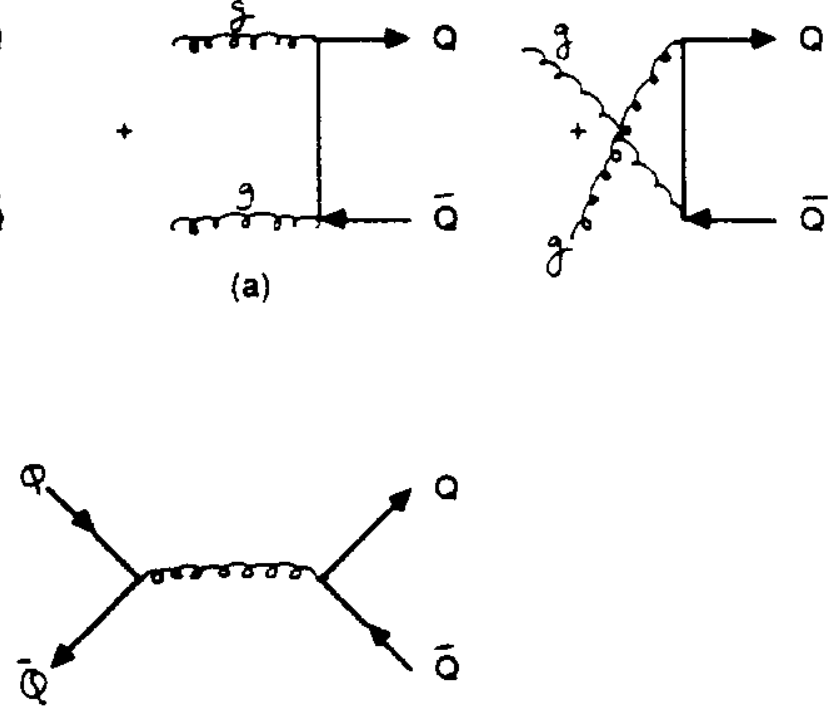

(b)

Figure 64: Lowest order Feynman graphs for the production of $Q \bar{Q}$ final state. a) Diagrams for gluon-gluon scattering b) Quark-antiquark scattering 
is obtained by the replacement $\hat{t} \leftrightarrow \hat{u}$. The last term in the above equation is due to the interference between $\hat{t}$ exchange and $\hat{u}$ exchange diagrams.

Similarly the differential cross section

$$
\frac{d \hat{\sigma}}{d \hat{t}}(q \bar{q} \rightarrow Q \bar{Q})=\frac{4 \pi \alpha_{s}^{2}}{9 \hat{s}^{2}}\left[\frac{\left(\hat{t}-m^{2}\right)^{2}+\left(\hat{u}-m^{2}\right)^{2}+2 m^{2} \hat{s}}{\hat{s}^{2}}\right]
$$

\subsubsection{Flavor excitation}

At high $Q^{2}$, the gluons in the nucleon can split into $Q \bar{Q}$ pairs giving an intrinsic heavy quark content to the nucleon. These $Q \bar{Q}$ pairs can be knocked out of the nucleon by $g Q \rightarrow g Q$ and $q Q \rightarrow q Q$ processes. This process will become less significant very fast as the mass of the heavy quark increases.

\subsubsection{Gluon splitting}

So far we have only considered first order processes that depend on $\alpha_{z}^{2}$. One can naively assume that higher order processes will be suppressed by corresponding powers of $\alpha_{s}$. However, since the $g g \rightarrow g g$ process is much more dominant than the process $g g \rightarrow q \bar{q}$, (see table 1), the second order process where one of the gluons splits into $g g \rightarrow Q \bar{Q}$ can become important[102].

One can show that

$$
\frac{\sigma(g g \rightarrow g Q \bar{Q})}{\sigma(g g \rightarrow Q \bar{Q})} \approx \frac{\sigma(g g \rightarrow g g)}{\sigma(g g \rightarrow Q \bar{Q})} \frac{\alpha_{s}}{3 \pi} \ln \frac{\hat{s}}{4 m^{2}}
$$

even though $\alpha_{g} / 3 \pi \approx 0.03, \sigma(g g \rightarrow g g) / \sigma(g g \rightarrow Q \bar{Q}) \approx 100$. Depending on $m$, the log term can add another factor of 2 or so leading to an enhancement of a factor of five to 10 in favor of the higher order process.

For top quark production, if $m_{t}<m_{W}$, then $W \rightarrow t \bar{b}$ will be a means of producing the top quark. And it is in this channel that UA1 reported their tentative signal. The branching ratio $W \rightarrow t \bar{b}$ will be smaller than the branching ratio $W \rightarrow u \bar{d}$ by the ratio of the phase space factors (ratio 
of the momenta of the top quark/ up quark in the rest frame of the W).

$$
\frac{2 p_{\text {top }}}{m_{W}}=\sqrt{\left[1-\frac{\left(m_{t}+m_{b}\right)^{2}}{m_{W}^{2}}\right]\left[1-\frac{\left(m_{t}-m_{b}\right)^{2}}{m_{W}^{2}}\right]}
$$

If $m_{t}<m_{Z} / 2$, then $Z \rightarrow t \bar{t}$ will contribute and the branching ratio will be suppressed by the factor $\sqrt{1-\left(4 m_{t}^{2}\right) /\left(m_{Z}^{2}\right)}$ from the canonical $Z \rightarrow u \bar{u}$ ratio.

The cross section for $t$ and $\bar{t}$ production due to $\mathrm{W}$ decay, $\mathrm{Z}$ decay and $t \bar{t}$ production are shown in Figure (65) as a function of the top mass [41], at CERN energies. It can be seen that $W$ decay dominates for $40<m_{t}<$ $75 \mathrm{GeV} / \mathrm{c}^{2} . \mathrm{Z}$ decay is negligible in terms of cross section .

\section{2 $b \bar{b}$ production and mixing}

We now review here the evidence for $b \bar{b}$ production and mixing reported recently by UA1 [103],[104].

\subsection{1 $b \bar{b}$ production}

In the absence of mixing, $b \bar{b}$ production and subsequent decay will yield

- Single muon events where one of the $b$ 's decays into a muon and the other decays hadronically.

- Opposite sign dimuon events where both $b$ 's and $\bar{b}$ 's decay into muons

- Same sign dimuon events where one of the $b$ 's cascades into a $c$ which decays into a $\mu^{+}$and the other $\bar{b}$ decays into another $\mu^{+}$. $\left(\mu^{-} \mu^{-}\right.$states result from the $\bar{b}$ cascading into a $\bar{c}$ etc.)

Along with $b \bar{b}$ production, we will have $c \bar{c}$ production which will lead to the first two of the above muon configurations. Figure (66) shows the $p_{t}$ spectrum of the muons in the single muon events and the opposite sign dimuon events. In order to insure that the dimuon spectrum comes from $b \bar{b}$ decay, one or both of the muons are demanded to be non-isolated. The full curves are predictions from Isajet [105] with all three processes for $b$ production 


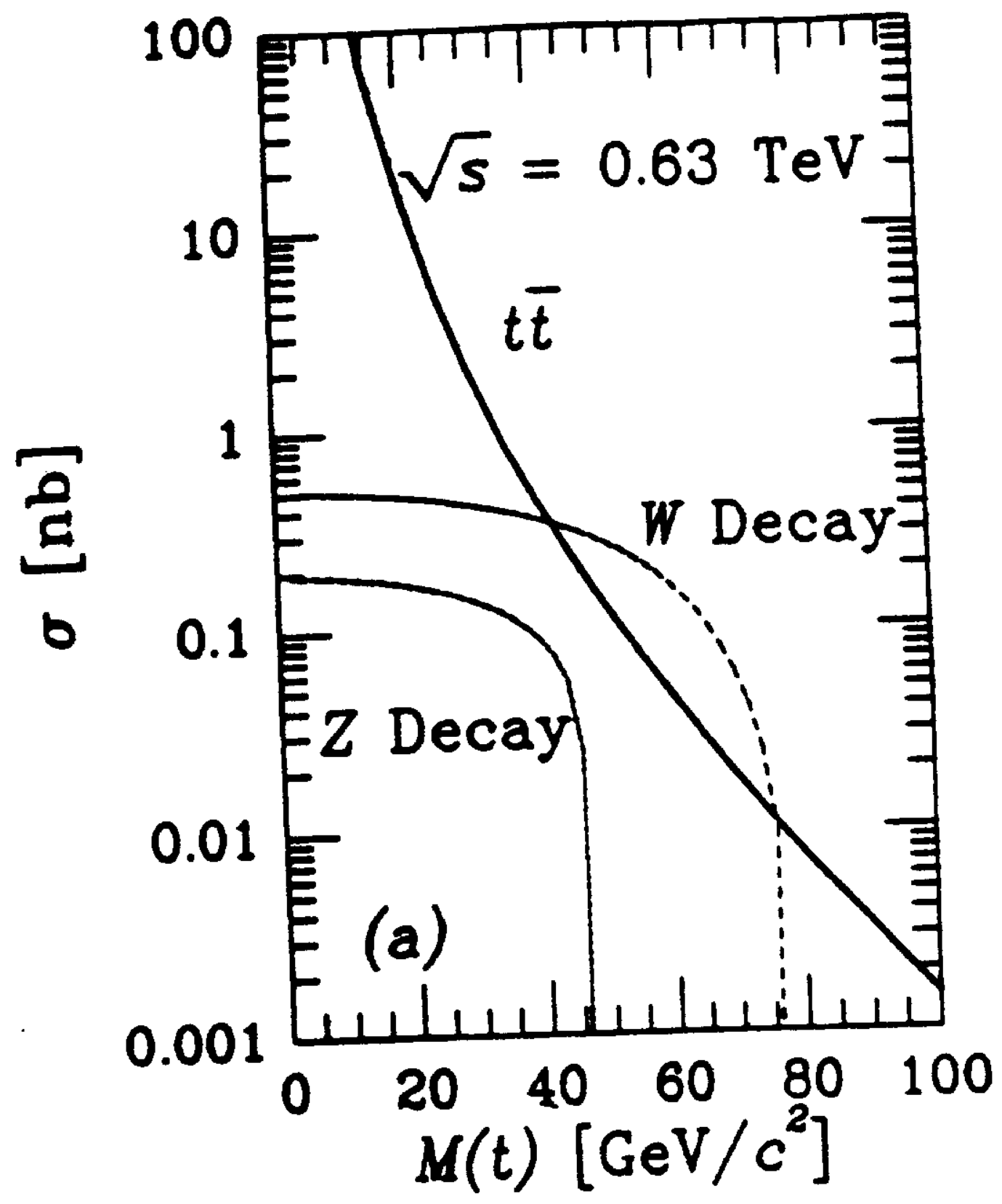

Figure 65: Cross section for top production at CERN energies from $W$ decay, $Z$ decay and pair production 
and $c$ production folded in. The dashed curves are the contribution from the lowest order processes only (namely flavor creation). It can be seen that the shape and the absolute magnitude of the muon cross section is understood in terms of the three production mechanisms outlined above. Figure(67) is the relative $p_{t}$ of the muon with respect to the jet axis for unlike sign dimuon events. The jet axis is defined by the charged particles in the jet, including the muon. Both the muons in the event enter this plot. The curves are a result of a two parameter fit of $c \bar{c}$ and $b \bar{b}$ components.

\subsection{2 $b \bar{b}$ mixing}

If however, there is a significant amount of $b \bar{b}$ mixing, the chance of a $b$ quark to turn into a $\bar{b}$ quark before decay is significant and subsequent decay will yield same sign dimuons. UA1 has just reported an excess of same sign dimuon events [104] which cannot be explained by the cascade mechanism discussed above. The predicted ratio of same sign to unlike sign dimuons in UA1 from the cascade process is $0.26 \pm 0.03$. The experimentally measured quantity is $0.42 \pm 0.07 \pm 0.03$. From this they deduce that the fraction of $b$ quarks that produce muons with a charge opposite to that which would be produced without mixing is $0.121 \pm 0.047$. This result preceded the recent announcement from ARGUS collaboration of observation of mixing in the $B_{d}^{0} \overline{B_{d}^{0}}$ system [106].

The introduction of high resolution vertex chambers will help clarify the physics processes involved in $b$ production since the $b$ quark has been measured to possess a relatively long lifetime. It will become possible to separate out the secondary vertex due to the $b$ decay and use this as an extra condition to isolate $b$ decay from competing sources of muons. Both CDF and DO experiments at the Tevatron as well as the upgraded UA1/2 detectors at CERN are expected to possess this ability.

\subsection{Evidence for top quark production}

In a paper entitled 'Associated production of an isolated large transverse momentum lepton (electron or muon) and two jets at the CERn $\bar{p} p$ collider' [101], the UA1 collaboration presented tenmtative evidence for the existence of the top quark in the mass range $30 \mathrm{GeV} / \mathrm{c}^{2}<m_{t}<50 \mathrm{GeV} / \mathrm{c}^{2}$. Their 


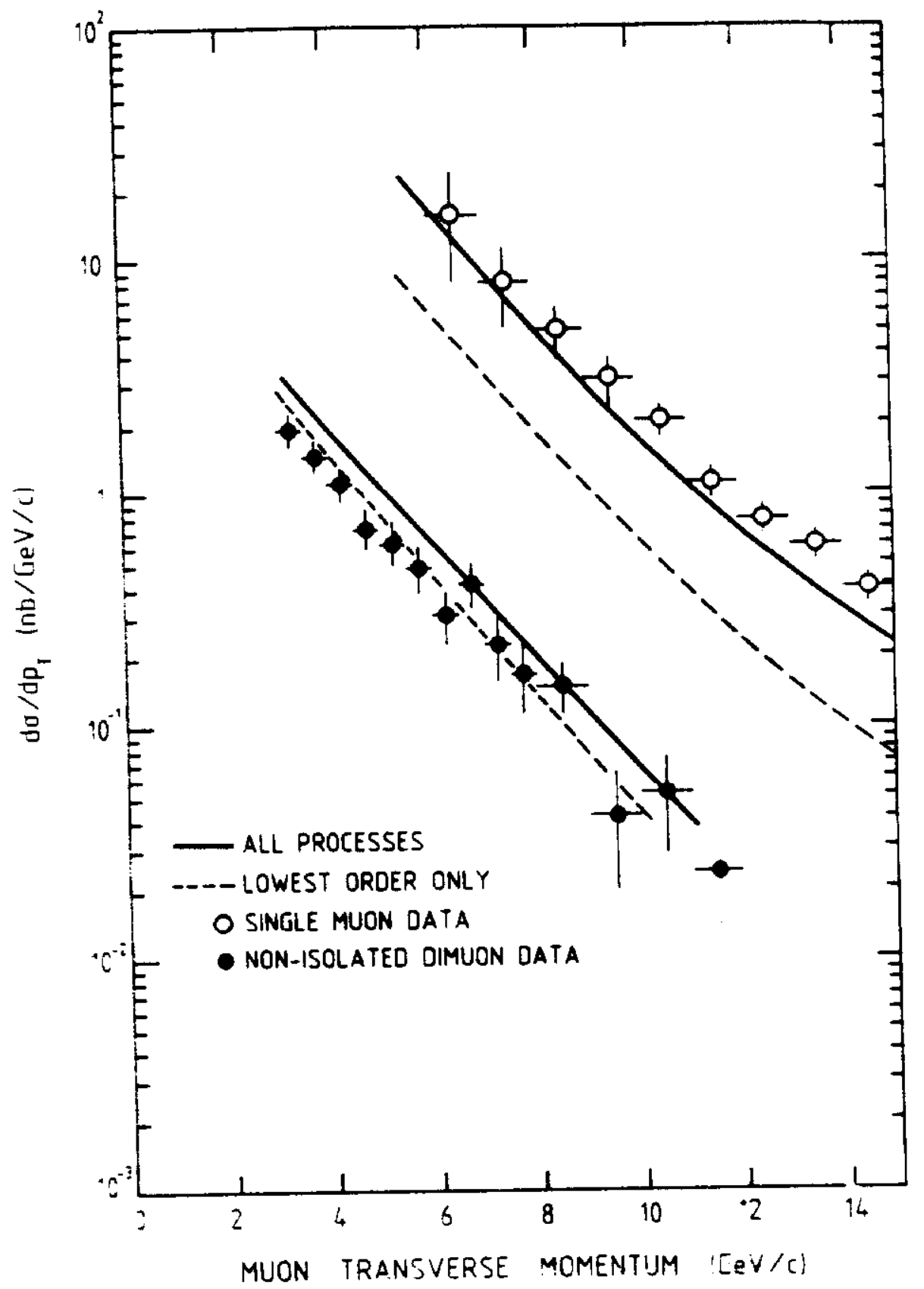

Figure 66: Inclusive muon $p_{t}$ distributions for single-muon events (open circles) and non-isolated dimuon events (solid circles). The curves are absolutely normalized QCD predictions from Isajet. The bwest order dashed curve uses flavor creation and the full curve contains all three mechanisms. 


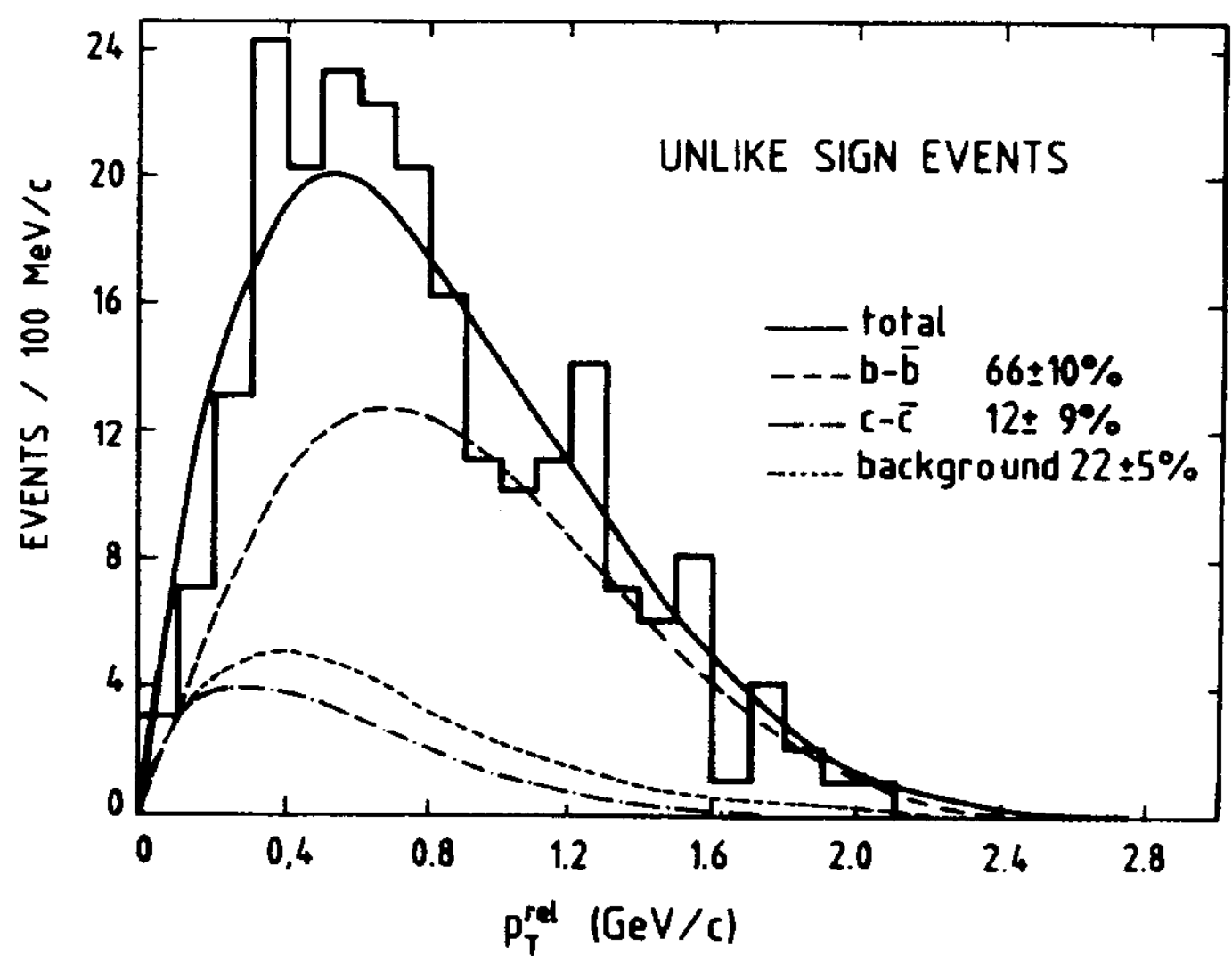

Figure 67: Transverse momentum of muons relative to the jet axis, which is defined by the charged particles in the jet including the muon 
argument ran as follows. If the top quark mass is less than $65 \mathrm{GeV} / \mathrm{c}^{2}$, the decay $W \rightarrow t \bar{b}$ will contribute significantly to the production of the top quark at CERN energies. See Figure (65) which shows the relative contributions from $W \rightarrow t \bar{b}$ and $t \bar{t}$ at CERN energies. Some fraction of the time the top quark will decay semi-leptonically as $t \rightarrow l b \nu$ where the detected lepton $l$ is an electron or a muon. Because $m_{t}-m_{b}$ is large, ( $m_{t}>22 \mathrm{GeV} / c^{2}$ from $e^{+} e^{-}$collisions.), the decay lepton will be thrown clear of the $b$ quark jet and will thus end up isolated a significant fraction of the time. So the potential signal should satisfy the following requirements

- The invariant mass of the $(b \bar{b} l \nu)$ system must peak around the W mass. The $\nu$ four vector must be replaced by its transverse component, which is all that is measured. This will broaden the spectrum somewhat.

- blv effective mass should cluster around a common value to be interpreted as the top quark mass. For $t$ quark production, the lepton should be associated with the $b$ jet which should in general be the second highest $E_{T}$ jet. The $\bar{b}$ jet results from the primary $\mathrm{W}$ decay and should thus be identified with the leading jet. (For $\bar{t}$ production from $W^{-}$production, all signs are reversed. We have suppressed Cabibbo rotation for the sake of clarity.)

In the published UA1 data sample, they had $68 \mathrm{~W} \rightarrow e \nu$ decays. Using the standard model branching ratios, they expect $181 \pm 20 \mathrm{~W} \rightarrow t \bar{b}$ decays for a top quark mass of $40 \mathrm{GeV} / \mathrm{c}^{2}$. (The phase space suppression of the decay is 0.71 for this mass). If one folds in the semi-leptonic branching ratio for $t \rightarrow l b \nu$ of $\approx 1 / 9$, one expects $20 \pm 2.2$ events of the above topology in the electron channel and a similar number in the muon channel. Folding in the requirement $E_{T}\left(\bar{b}_{j e t}\right)>8 \mathrm{GeV}$ and $E_{T}\left(b_{j e t}\right)>7 \mathrm{GeV}$ and $p_{t}(l)>12 \mathrm{GeV}$, the expectation is $4 \pm 0.3$ events for each leptonic channel. This is before further geometrical and track isolation cuts.

UA1 then finds 3 such events in the electron channel and 3 in the muon channel. Figure (68) is a plot of such an electron +2 Jet event on the interactive graphical display. Figure (69) is the scatterplot of the 4 body effective mass $m\left(l \nu_{T} J_{1} J_{2}\right)$ vs. $m\left(l \nu_{T} J_{2}\right)$ for these events. There is clustering of the four body mass around theW mass and the 3 body mass clusters 

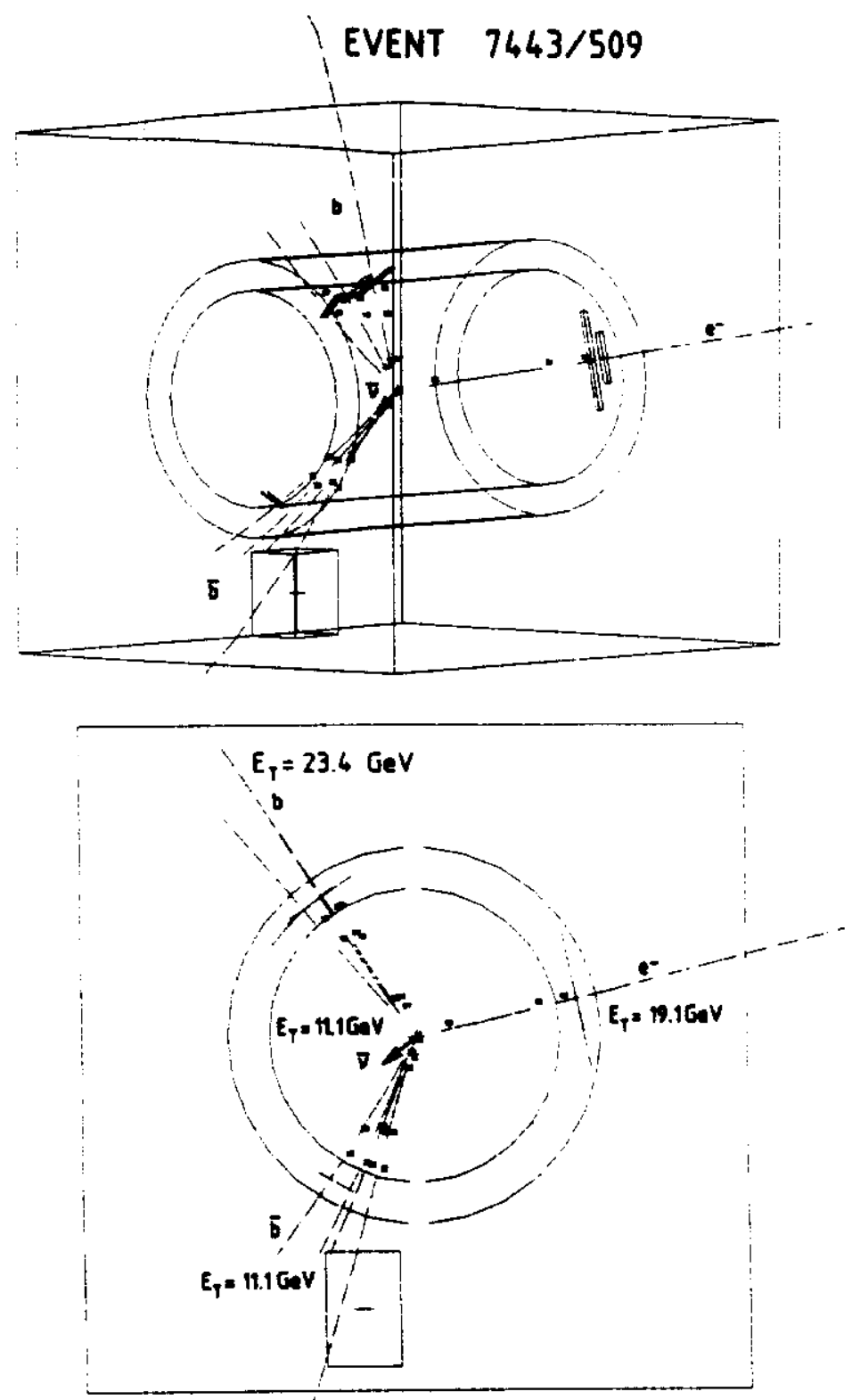

Figure 68: A picture of a typical electron +2 jet events possessing the top topology on the interactive display 


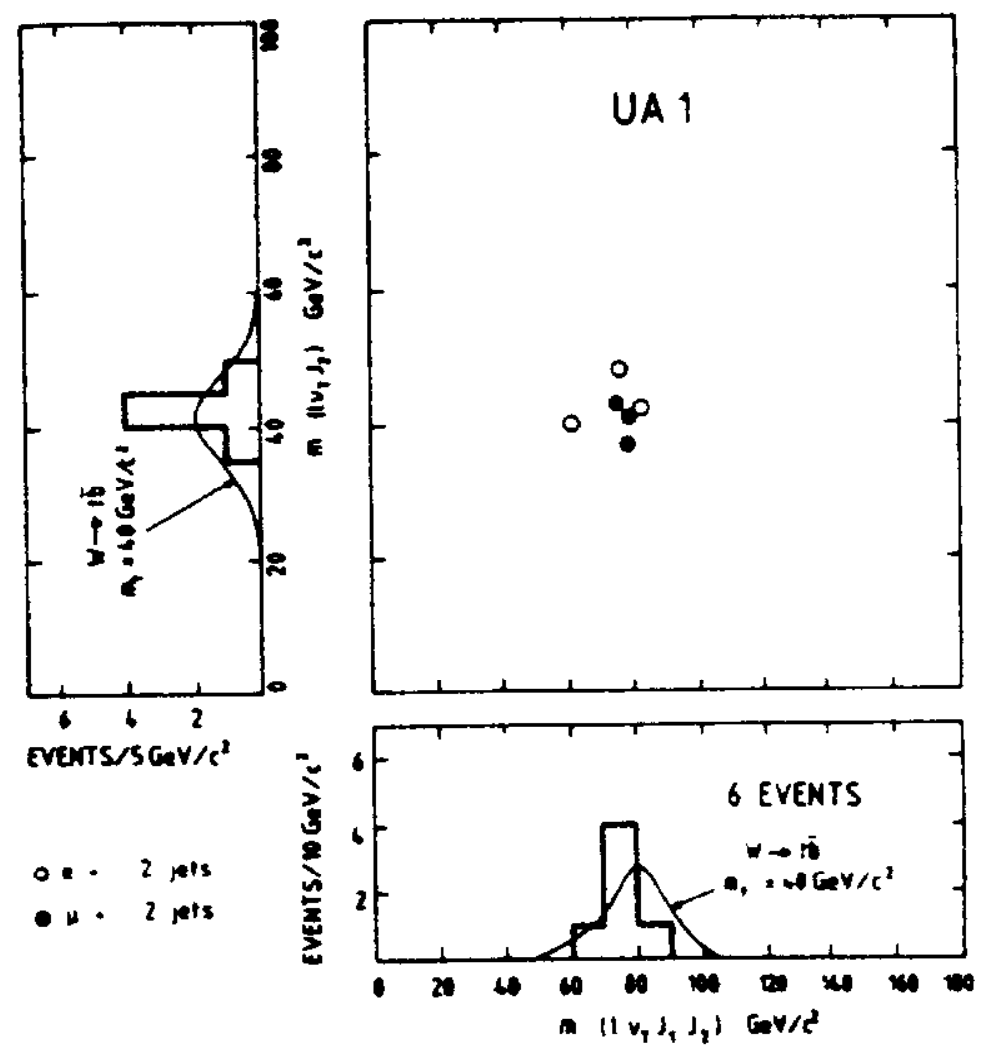

Figure 69: Scatterplot of the 4 body effective mass $l \nu_{T} J_{1} J_{2}$ vs 3 body effective mass $l \nu_{T} J_{2}$. The four body mass peaks at the $W$ mass and the three body mass clusters around a common value 
around a value of $40 \mathrm{GeV} / \mathrm{c}^{2}$. This is true for both the muon and electron events. Because of the systematic uncertainties involved in jet reconstruction, UA1 sets a mass range of $30-50 \mathrm{GeV} / \mathrm{c}^{2}$ for the top quark, if the events observed be indeed due to such an object.

Much of the paper is taken up in proving that the observed topology of lepton +2 Jets cannot be due to other known sources of background. A thorough and fairly convincing argument is made that the events observed cannot be due to QCD 3-jets (the third jet from initial state bremsstrahlung) faking the electron +2 jet sample with one of the jets fragmenting in such a way as to fake an electron. The argument is based on the observation that 1) the angular distribution of the third jet will be strongly peaked in the beam direction since it is expected to be due to gluon bremsstrahlung and 2) the jet faking the electron will have small $E_{T}^{\text {out }}$ where $E_{T}^{\text {out }}$ is defined as the energy component perpendicular to the plane formed by the beam axis and the highest $E_{T}$ jet. The probability of the QCD background faking the observed events was quoted as $5.2 \times 10^{-4}$.

UA1 does another background analysis in the muon channel and concludes that the background from $b \bar{b} g$ final state in the observed events is $1 \%$. It is assumed that this final state background calculation includes contributions from gluon splitting. There has, however been no further information from UA1 on this subject despite the fact that much additional data has been in hand. There has been an internal UA1 note [107] questioning the validity of the published top analysis. The lectures given by Dave Cline in this school contain data which amount to a new lower limit for the top quark mass.

There exist potentially other channels for searching for the top quark. If $m_{t}<m_{W}, W \rightarrow t \bar{b}$ will result in like sign dileptons from the $t$ and $\bar{b}$ decay. With increased luminosities at CERN and higher energies at Fermilab, this channel with $\mu^{+} \mu^{+}, \mu^{+} e^{+}$, and $e^{+} e^{+}$final states (and their charge conjugates) should be observable. The lepton from the top decay will be isolated. This is potentially separable from the $b \bar{b}$ mixing which will yield two non-isolated leptons. For a top quark mass in the neighborhood of $40 \mathrm{GeV} / \mathrm{c}^{2}, \eta_{t}$ production and decay into two photons is potenitally observable at Tevatron energies.[108]. Both photons will be isolated and the two photon effective mass will cluster around the $\eta_{t}$ mass enabling one to 
eliminate potential backgrounds from QCD hard photon emissions.

\subsection{Scenario for the Tevatron}

If $m_{t}>65 \mathrm{GeV} / c^{2}, \mathrm{~W}$ decay will become insignificant as a source of top quarks. $Z \rightarrow t \bar{t}$ will not contribute either. This will eliminate SLC and LEP I as the machines likely to discover the top quark in favor of the Tevatron. Figure (70) shows the $t \bar{t}$ cross section as a function of the top quark mass for the $s \bar{p} p s$ and the Tevatron energies[41]. It is clear that the greater the top quark mass, the greater the advantage the Tevatron has over $s \bar{p} p s$. One can phrase this argument in a slightly different way. At any $\sqrt{s}$ and integrated luminosity, one can define the discovery limit for finding the top quark as that mass at which 100 top quarks are produced in the experiment. If the mass is higher, fewer than 100 events will be produced for that integrated luminosity, and one can say (somewhat arbitrarily) that the top will not be discovered. Figure(71) shows the discovery limit as a function of the integrated luminosity in the range $\left[0-1 p b^{-1}\right]$ for $s \bar{p} p s$ and Tevatron energies. It is clear that with an integrated luminosity of $1 p b^{-1}$ , CERN can see a top quark if it less massive than $\approx 50 \mathrm{GeV} / \mathrm{c}^{2}$. We are only considering $t \bar{t}$ production for this discussion. $1 p b^{-1}$ is where UA1 is at present. At the Tevatron, we can reach a discovery limit of $50 \mathrm{GeV} / \mathrm{c}^{2}$ with $100 b^{-1}$ luminosity. With $1 p b^{-1}$, one can reach a limit of $\approx 90 \mathrm{GeV} / \mathrm{c}^{2}$.

Figure (72) is a continuation of the same argument in the luminosity range $1 p b^{-1}-30 p b^{-1}$. This is perhaps the total integrated luminosity likely to be achieved at CERN and FNAL in the next five years. At $30 \mathrm{pb}^{-1}$, CERN discovery limit is at $\approx 90 \mathrm{GeV} / \mathrm{c}^{2}$ whereas FNAL can reach up to $\approx 170 \mathrm{GeV} / \mathrm{c}^{2}$.

To conclude, the status of the top quark is highly uncertain. If the decay $Z \rightarrow t \bar{t}$ is forbidden, the Tevatron has a unique chance of finding the object. This will however, require a careful and methodical understanding of all the background processes. 


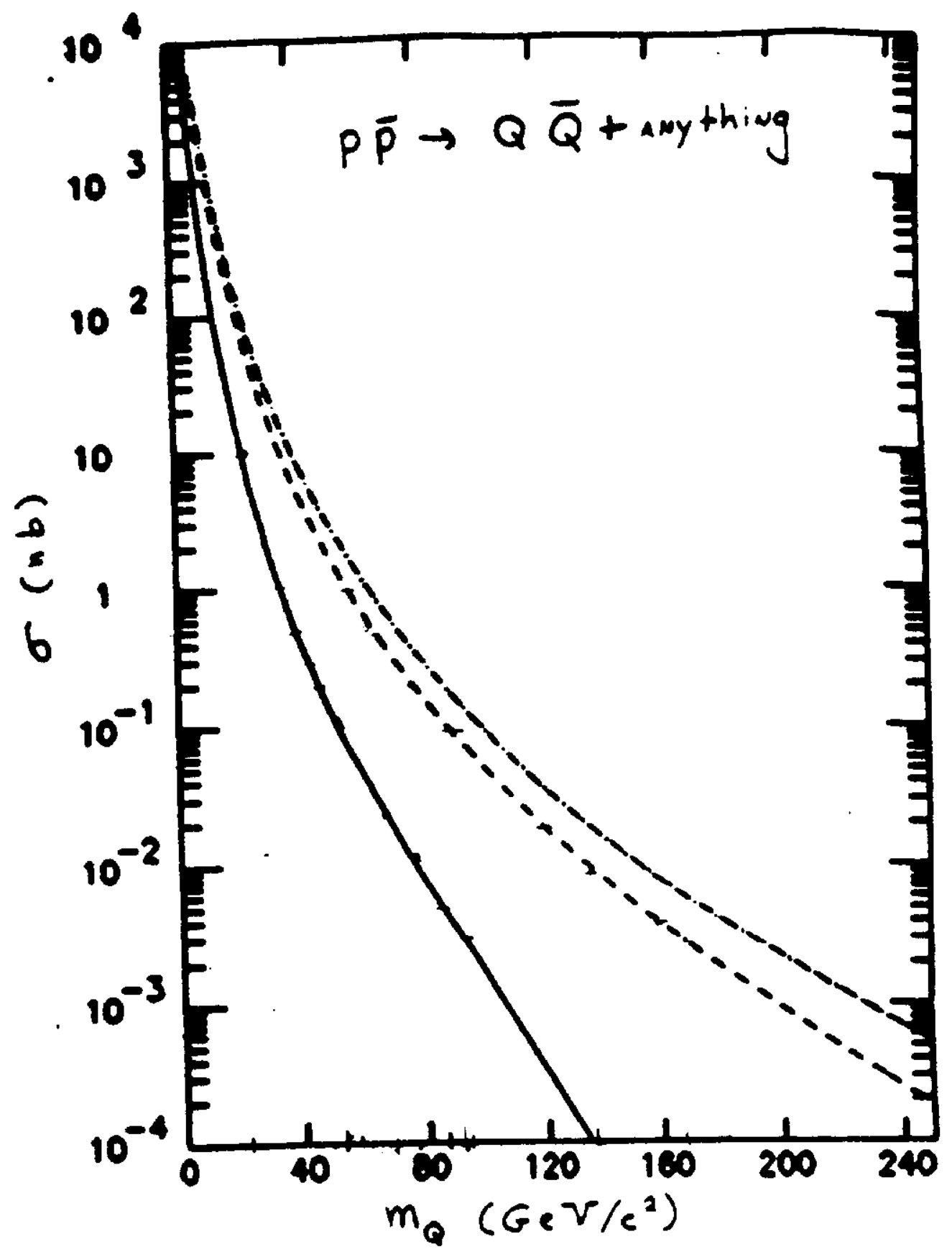

Figure 70: The total cross section for heavy quark pair production as a function of heavy quark mass at $630 \mathrm{GeV}$ (solid line), $1.8 \mathrm{TeV}$ (dashed line) and $2.0 \mathrm{TeV}$ (dot-dashed line) 


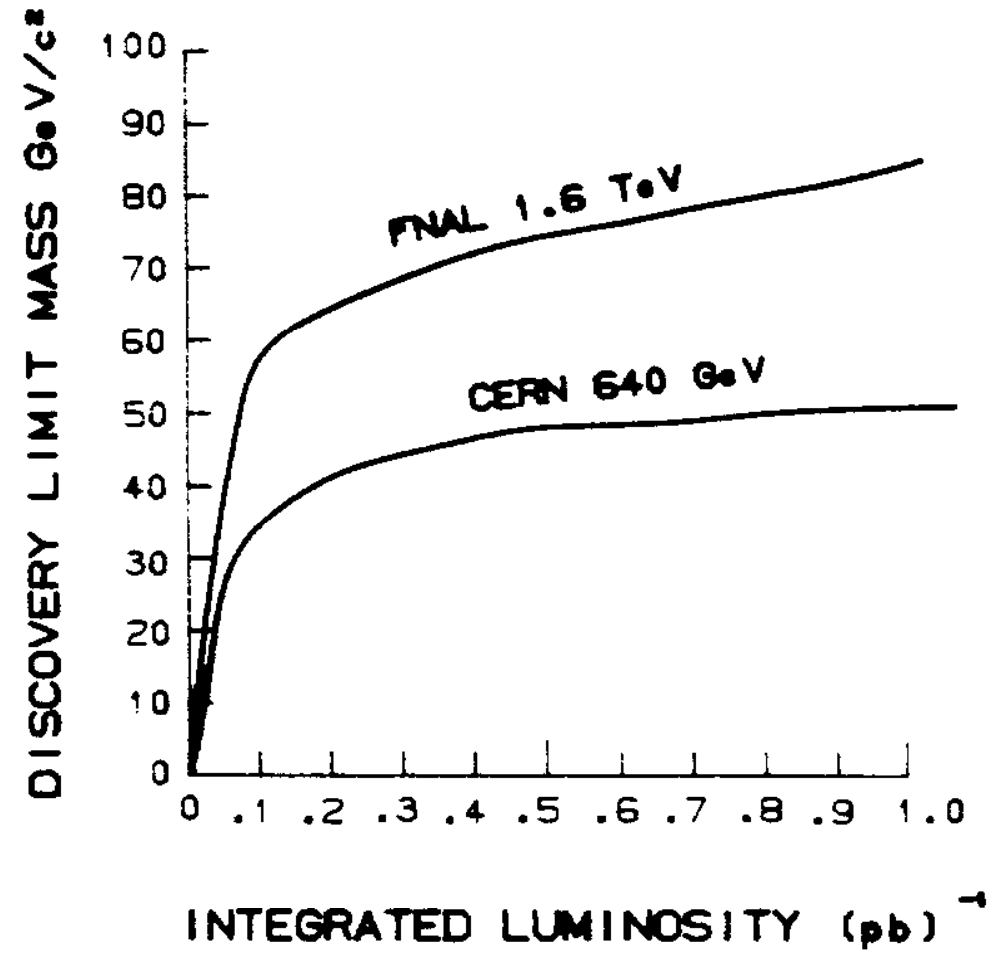

Figure 71: The discovery limit for top quark mass as a function of integrated luminosity in the range $0-1 p b^{-1}$ at CERN and Fermilab energies. 


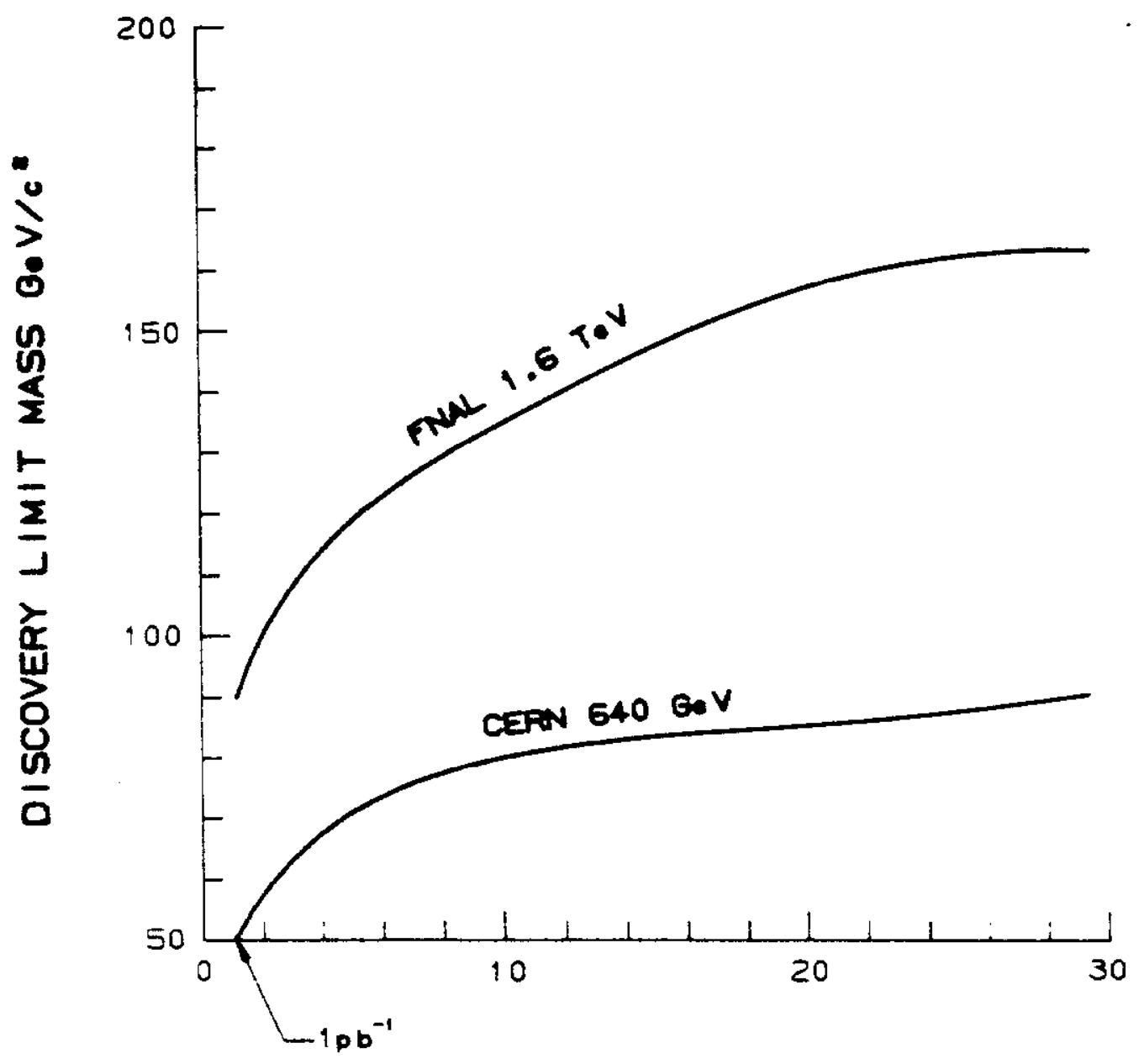

INTEGRATED LUMINOSITY

Figure 72: The discovery limit for top quark mass as a function of integrated luminosity in the range $1-30 p b^{-1}$ at CERN and Fermilab energies. 


\section{Physics beyond the standard model}

\subsection{Missing $E_{T}$ measurements}

Calorimeters which have coverage over the full solid angle can detect most of the energy emitted in an interaction. Such calorimeters are known in the jargon as hermetic calorimeters. No calorimeter can be perfectly hermetic since room must be left for the beam pipe to bring in the particles that interact. The beam pipe will permit a substantial amount of the energy of the interaction to escape undetected. If the interaction contains weakly interacting final states (neutrino, photino etc.) there will be missing energy in the event. The longitudinal component of the missing energy will be poorly measured because of particles escaping down the beam pipe as well as due to fluctuations in the measuring process. A large missing transverse energy, however, will in general be detectable. It was by demanding a high $p_{t}$ electron and missing transverse momentum that the first $W$ signal was observed.

Missing $E_{T}$ thus provides a powerful tool for detecting particles that are weakly interacting. Theories that go beyond the standard model, e.g. supersymmetry, contain particles that are weakly interacting (e.g. photino, sneutrino). Hence the tremendous interest in missing $E_{T}$ physics. The ability of a calorimeter to measure missing $E_{T}$ will depend critically on two factors.

- The average overall energy resolution of the calorimeter. Clearly, the better the resolution, the better the ability to measure missing $E_{T}$. In order to obtain good resolution, it is necessary to build the calorimetry out of materials that yield good intrinsic resoultion [109] (e.g. Uranium Liquid Argon). It is also necessary to calibrate the calorimeter accurately and maintain the calibration during the lifetime of the experiment.

- The homogeneity of the calorimeter. Due to practical reasons, no calorimeter can be made perfectly homogeneous in its resolution. Cracks between modules and dead material from support structures will introduce inhomogeneities and cause tails to build up in missing 
$E_{T}$ distributions. If the only signature in the event is the missing $E_{T}$, and one is looking for rare processes, these inhomogeneities have to be kept to a minimum. [110].

\subsection{Search for Supersymmetry}

Supersymmetry or SUSY, proposed by Wess and Zumino [111] requires a partner to every elementary particle such that for every ordinary boson there corresponds a supersymmetric fermion and vice versa. So for the gluon, quark and photon, there correspond supersymmetric partners, the gluino, squark and photino respectively. The gluino and photino are fermions and the squark is a scalar particle. Only the spin is thus affected. In the limit of exact supersymmetry, all other quantum numbers are identical between a particle and its SUSY partner. Supersymmetry is clearly not an exact symmetry since at present energies, we have not yet been able to observe any SUSY particles, which implies that the masses of the superpartners have to be larger their corresponding ordinary particles. Supersymmetry breaking is thought to occur in such a way that only the masses are affected, all other quantum numbers remaining the same. The lightest SUSY particle is thought to be absolutely stable. If this is the photino or the sneutrino, it will escape detection and will yield missing $E_{T}$ events.

\subsection{Experimental situation}

The UA1 experiment, fixed target experiments as well as $e^{+} e^{-}$experiments [112] have conducted SUSY searches. Despite the initial optimism of the UA1 monojet paper, all present data point to null results. Figure (73) gives the best UA1 limits obtained on the gluino and the squark masses from the monojet analysis [113]. The mass limits for the gluino and the squark are coupled. Taken individually, $M_{\tilde{q}}>70 \mathrm{GeV} / \mathrm{c}^{2}$ at $90 \%$ confidence level irrespective of the gluino mass and $M_{\tilde{g}}>60 \mathrm{GeV} / \mathrm{c}^{2}$ irrespective of the squark mass at $90 \%$ confidence level. A light gluino window for a gluino mass between 3 and $5 \mathrm{GeV} / \mathrm{c}^{2}$ cannot as yet be excluded.

For the photino, three separate mass windows are of interest. 


\section{LIMITS ON GLUINO AND SOUARK MASS}

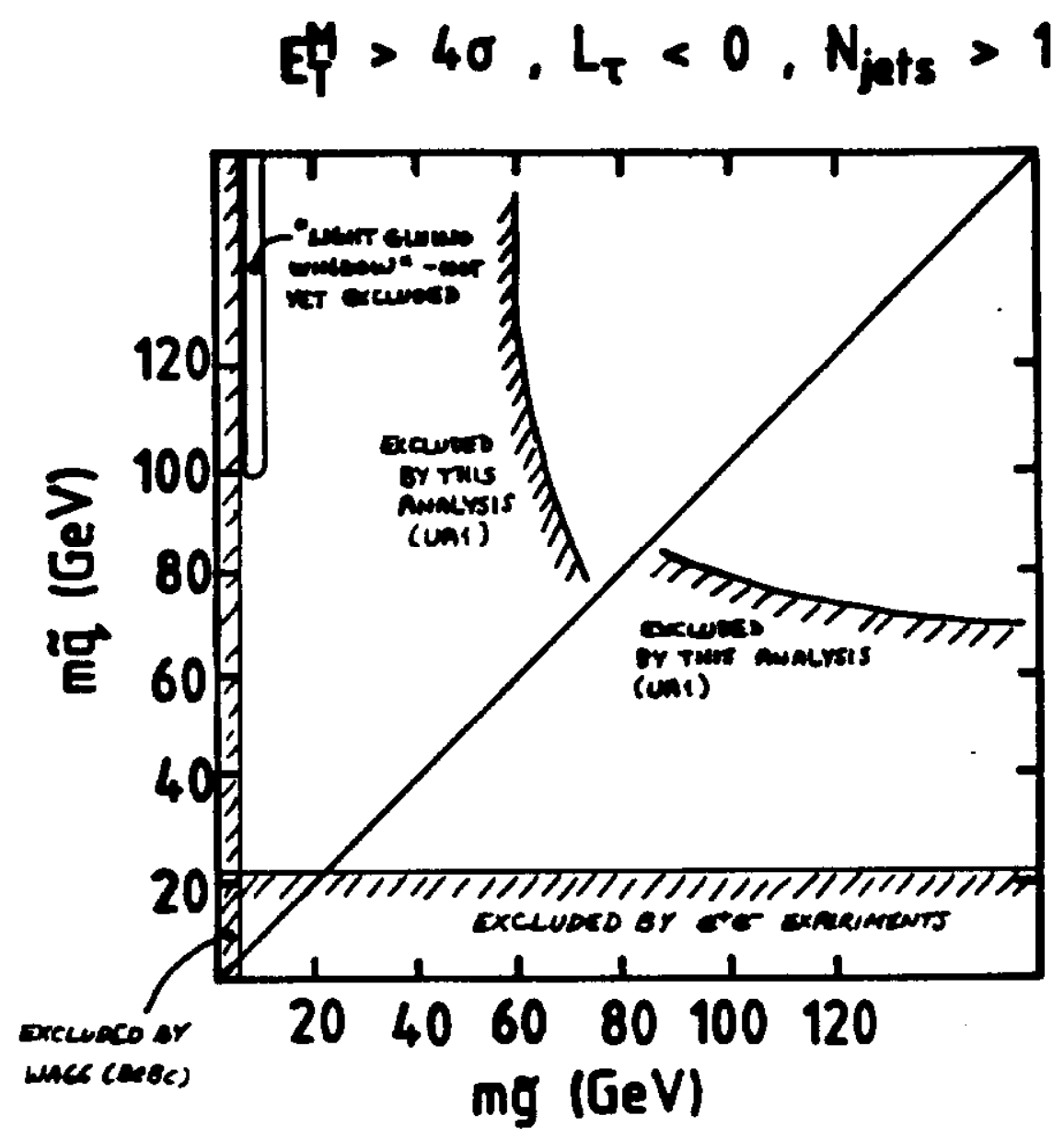

Figure 73: Mass limits for the Squark and the Gluino 
- Photino is the lightest super-partner and its mass is less than $1 \mathrm{MeV} / \mathrm{c}^{2}$.

- Photino is the lightest super-partner and its mass is greater than $1 \mathrm{MeV} / \mathrm{c}^{2}$.

- Photino decays into a photon and a Goldstino.

In the first case, photinos are stable spin $1 / 2$ particles. An upper limit on the photino mass of $100 \mathrm{eV} / \mathrm{c}^{2}$ results from demanding that the mass density of the universe be less than the closure density [114]

In the second case, Goldberg [115] has pointed out that photino pairs can annihilate into ordinary fermion pairs by the exchange of an sfermion. This leads to a sfermion mass dependent upper bound on the mass of the photino. For sfermion masses less than $100 \mathrm{GeV} / \mathrm{c}^{2}$, the photino mass has to be less than $\approx 20 \mathrm{GeV} / \mathrm{c}^{2}$.

The third case ensues if a massless Goldstino exists. The photons produced from photino decays must have thermalized with the cosmic microwave background [116]. This requires that the photino lifetime is less than 1000 seconds and that the photino mass be greater than $1.75 \mathrm{MeV} / \mathrm{c}^{2}$, if the supersymmetry breaking scale is of the order of $1 \mathrm{TeV}$. See [41] for a comprehensive review of these limits and experiments.

Figure (74) shows the total cross section for gluino pair production as a function of gluino mass for $s \bar{p} p s$ and Tevatron energies. The lower solid line gives the rates for center of mass energy of $\sqrt{(s)}=630 \mathrm{GeV}$, the middle solid line for $\sqrt{(s)}=1.8 \mathrm{TeV}$, and the upper solid line for $\sqrt{(s)}=$ $2.0 \mathrm{TeV}$, with squark mass set at $1 \mathrm{TeV}$. The dashed line shows the rates at $\sqrt{(s)}=630 \mathrm{GeV}$ for the squark mass equal to the gluino mass. The absolute rapidity of each of the gluinos is less than 1.5 . It can be seen that for an integrated luminosity of $10 \mathrm{pb}^{-1}$, the discovery limit (100 events produced in the experiment) for gluinos is $\approx 180 \mathrm{GeV} / \mathrm{c}^{2}$ at the Tevatron. and $\approx 90 \mathrm{GeV} / \mathrm{c}^{2}$ at $\mathrm{CERN}$.

Figure (75) shows the total cross section for up squark production as a function of the squark mass for $s \bar{p} p s$ and Tevatron energies. The bottom solid line gives the rates for $\sqrt{(s)}=630 \mathrm{GeV}$, the middle solid line gives the rates for $\sqrt{(s)}=1.8 \mathrm{TeV}$ and the top solid line gives the rates 


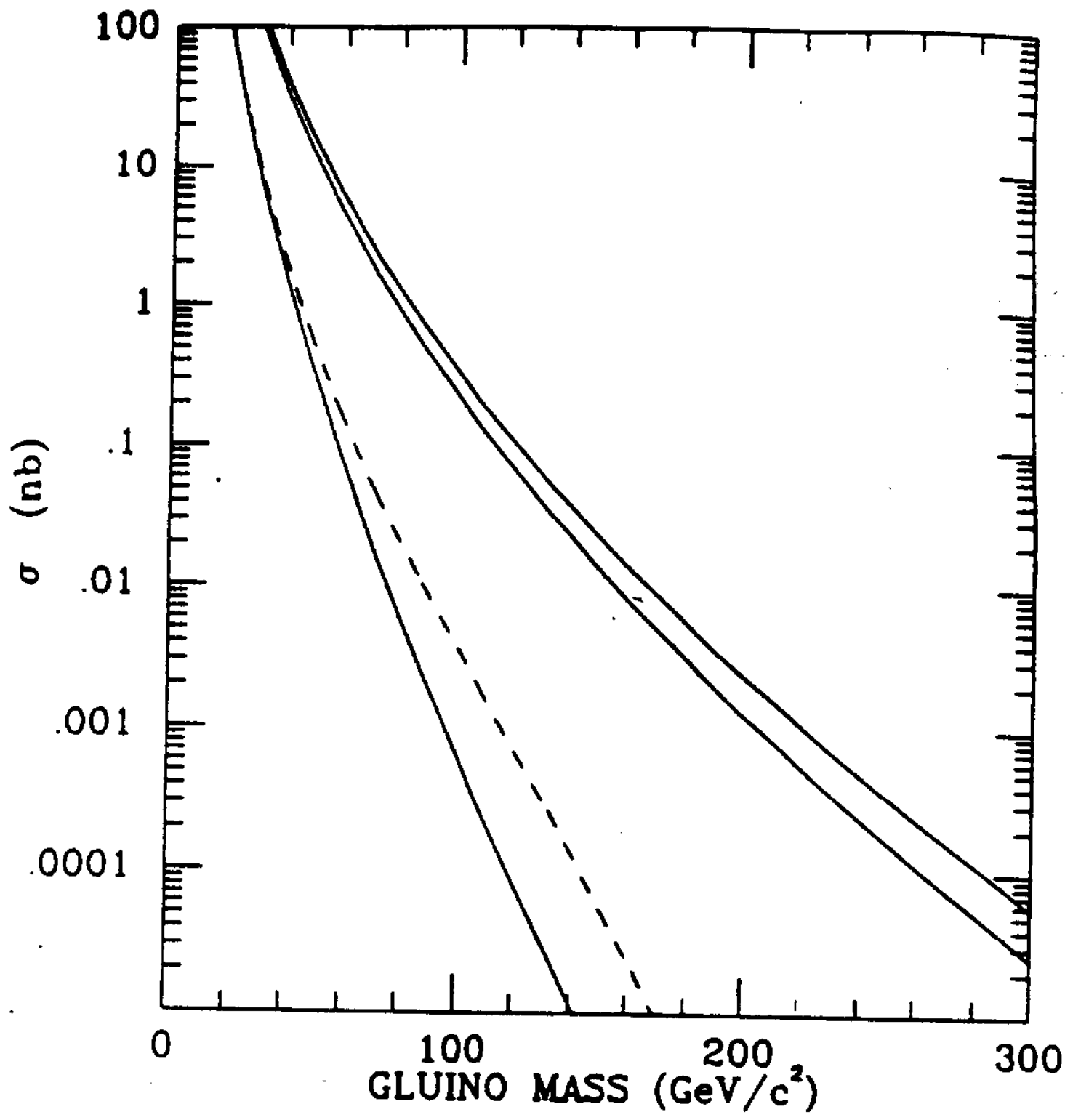

Figure 74: The total cross section for gluino pair production as a function of gluino mass. See text for explanation of curves 
for $\sqrt{(s)}=2.0 \mathrm{TeV}$, with the gluino mass equal to the squark mass. The dashed line gives the rate for $630 \mathrm{GeV}$ with the gluino mass equal to $1 \mathrm{TeV} / \mathrm{c}^{2}$. The absolute rapidity of the up squark and the associated antisquark are restricted to less than 1.5 . The corresponding discovery limits are $\approx 160 \mathrm{GeV} / \mathrm{c}^{2}$ and $\approx 90 \mathrm{GeV} / \mathrm{c}^{2}$ at the Tevatron and CERN respectively.

Figure (76) shows the total cross section for photino production and a light squark (up or down) as a function of photino mass. It is assumed that the photino mass is equal to the up squark and down squark mass. The lower solid line is for $\sqrt{(s)}=630 \mathrm{GeV}$, the upper solid line is for $\sqrt{(s)}=$ $1.8 \mathrm{TeV}$ and the dashed line for $\sqrt{(s)}=2.0 \mathrm{TeV}$. The absolute rapidities of both the photino and the squark are less than 1.5. The discovery limits are $\approx 65 \mathrm{GeV} / \mathrm{c}^{2}$ for FNAL and $\approx 45 \mathrm{GeV} / \mathrm{c}^{2}$ for CERN. These figures are taken from Eichten [41]. The discovery limits are quoted to give the reader an idea of the increased potential the higher energy of the Tevatron yields. Having produced $\approx 100$ events in the experiment, it is yet another matter to analyze the events to produce a convincing signal that is not overwhelmed by backgrounds.

\subsection{Technicolor}

Technicolor was yet another scheme proposed to overcome the unnaturalness of the standard model at high energies. The simplest model was proposed by S.Weinberg [117] and L.Susskind [118]. This scheme, known as minimal technicolor, introduces a new set of fermions (technifermions) interacting via a new non-abelian gauge interaction (technicolor) usually taken to be $\mathrm{SU}(4)$ invariant. This model however does not provide a mechanism for generating masses for ordinary quarks and leptons. To remedy this, Extended Technicolor models have been proposed [119]. The idea is to embed the technicolor group into a larger 'extended technicolor group' which couples quarks and leptons to technifermions. With the extension of supersymmetry to superstring models, technicolor seems to have fallen out of vogue with the theorists on grounds of theoretical elegance. However, the experimentalist must remain open to reasonable theoretical alternatives when exploring unchartered territory. 


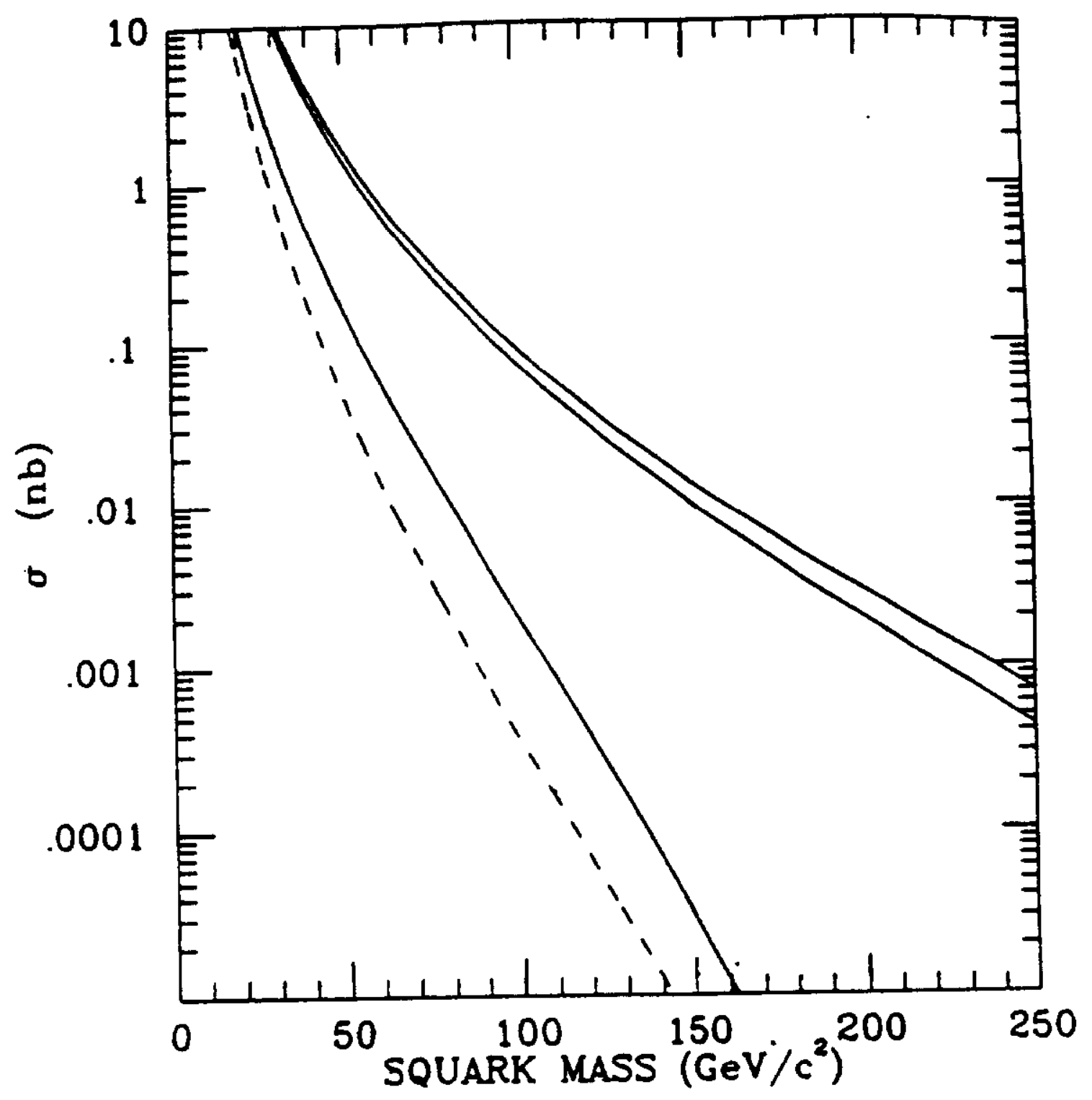

Figure 75: Total cross section for up squark production in $\bar{p} p$ collisions as a function of up squark mass. See text for an explanation of the curves 


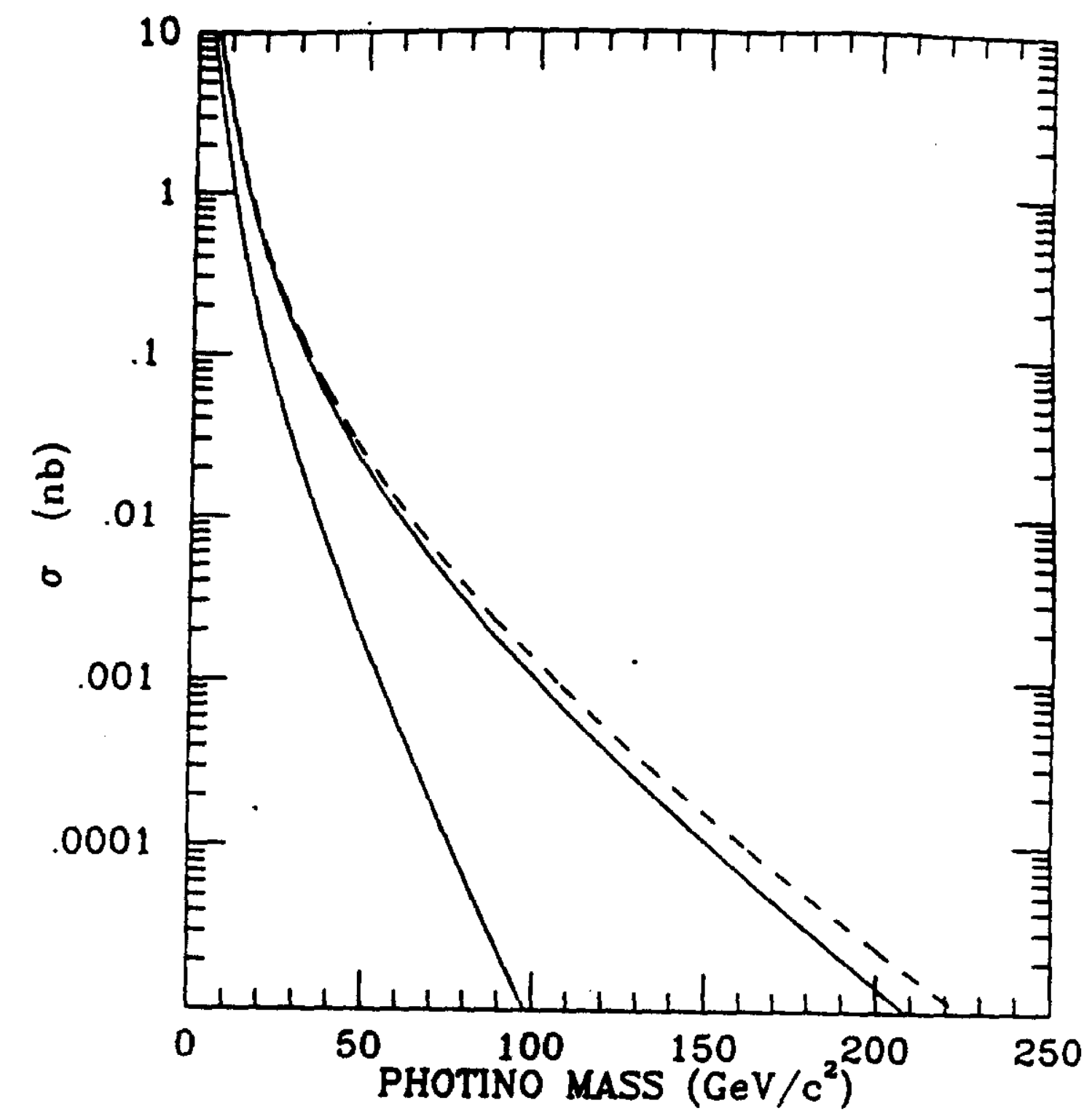

Figure 76: Total cross section for the associated production in $\bar{p} p$ collisions of a photino and a light squark (up or down) as a function of the photino mass. See text for an explanation of the curves 
Due to the breakdown of chiral symmetry, technipions will be formed with masses much below the technicolor mass scale and some of them may even be light enough to be produced at the Tevatron. There are 63 such goldstone bosons in the Extended Technicolor scheme of which 56 are colored and 7 are color neutral.

Figure (77) shows the production cross section at $y=0$ for a color singlet technipion $P^{0^{\prime}}$ in $\bar{p} p$ collisions as a function of the technipion mass [43]. The solid curve is for $\sqrt{(s)}=2 \mathrm{TeV}$, the dasehd curve is for $\sqrt{(s)}=1.6 \mathrm{TeV}$ and the dotted curve is for $\sqrt{(s)}=630 \mathrm{GeV}$. The principle decays of $P^{0^{\prime}}$ are into $g g, b \bar{b}$ and $\tau^{+} \tau^{-}$.

Figure (78) gives the production cross section at $\mathrm{y}=0$ for the color octet technipion $P_{8}^{0}$. The solid curve is for $\sqrt{(s)}=2 \mathrm{TeV}$, the dasehd curve is for

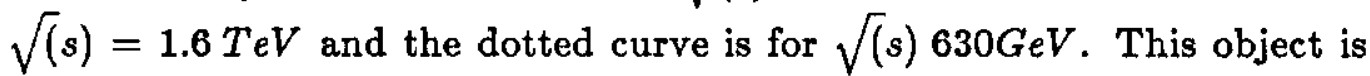
much more copiously produced than the color singlet state since $g g$ fusion can occur with any pair of gluons. Corresponding discovery limits are larger and the Tevatron has a good chance of establishing the existence of this object, given the validity of the extended technicolor scheme.

\subsection{Higgs Searches}

Figure (79) shows the three dominant mechanisms for Higgs production in $\bar{p} p$ collisions. Of these, the mechanism (c), where the Higgs is formed by $W W$ fusion only becomes important for Higgs masses $>2 M_{W}$ and hence is perhaps more relavant for the SSC, rather than the Tevatron. The other two mechanisms are dominated by the heaviest quark accessible since Higgs couplings to quarks increase with the mass of the quarks. If the top quark mass is not too large for the top to be produced at the Tevatron, then diagrams (a) and (b) with top quarks coupling to the Higgs will result in Higgs production at the Tevatron. However, the produced Higgs particles will, by the same token, decay predominantly into $t \bar{t}$. This final state will be completely swamped by the $t \bar{t}$ from $g g$ fusion. The Higgs signal then has to be looked for in rare decay modes which in turn demand higher production cross sections than are available at the Tevatron. The SSC is thus a far better accelerator for a successful Higgs search. Quoting numbers for Higgs 


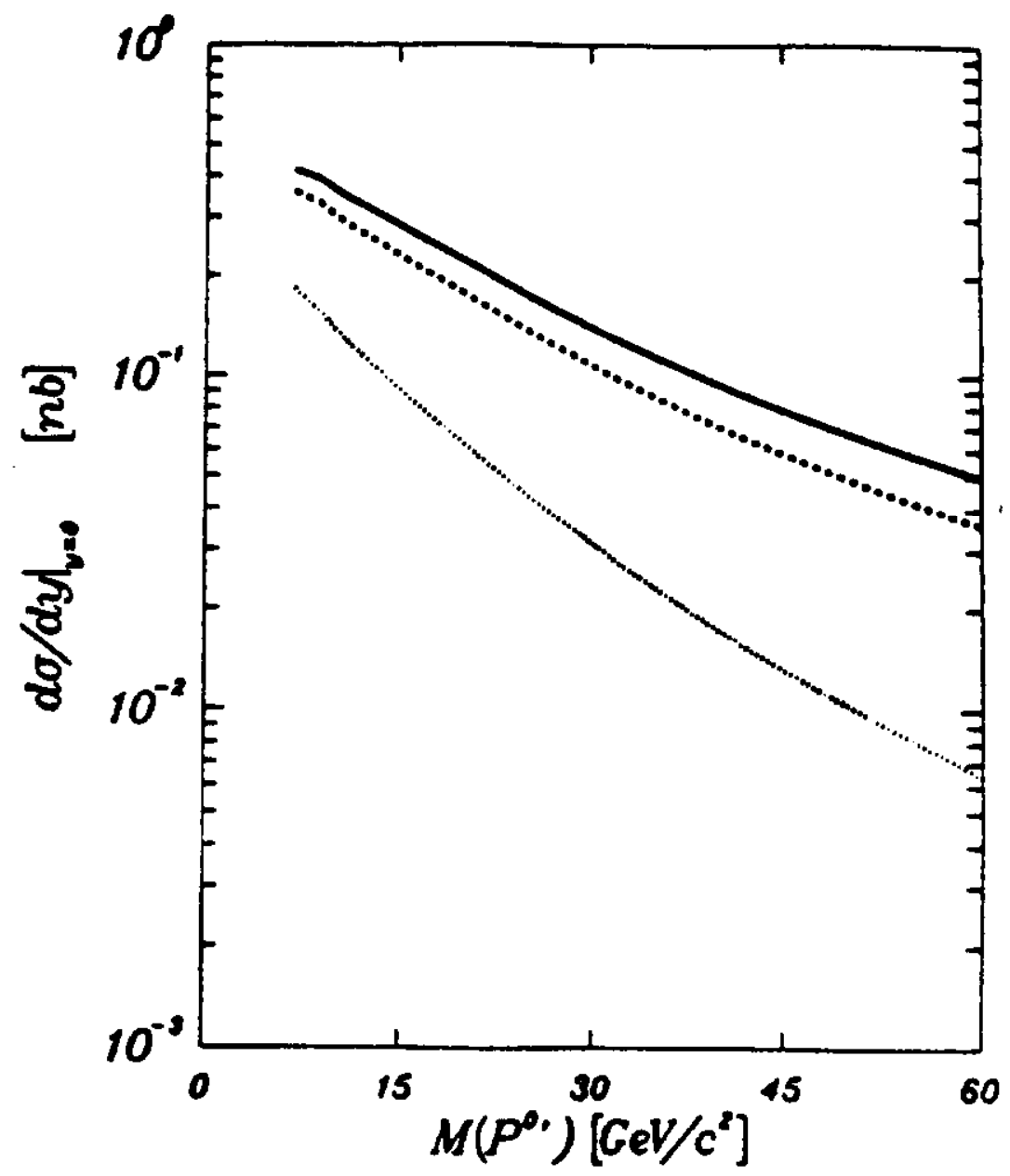

Figure 77: Differential cross section for the production of the color singlet technipion $P^{\prime 0}$ at $y=0$ in $\bar{p} p$ collisions. See text for an explanation of the curves 


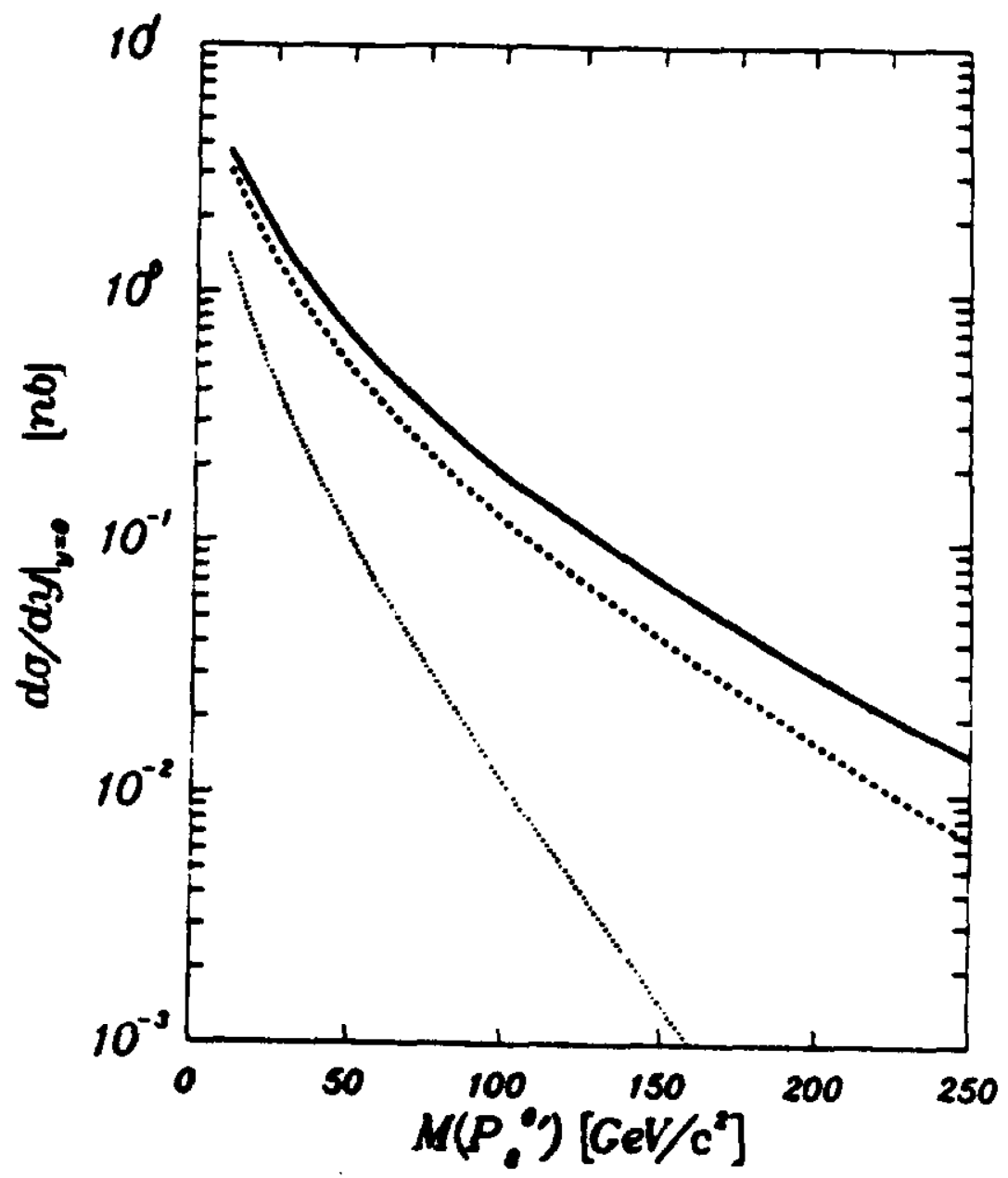

Figure 78: The differential cross section for the production of the color octet technipion $P_{8}^{0}$ at $y=0$ in $\bar{p} p$ collisions. See text for an explanation of the curves 
cross sections is a risky business, since they are strongly coupled to the mass of the Higgs and that of the top quark, both of which are unknown as of this writing. For Tevatron energies, diagram (a) is negligible since intrinsic top-quark structure function strengths are small. Diagram (b) results in a total cross section via gluon fusion of $3 p b$ for a Higgs mass of $100 \mathrm{GeV} / \mathrm{c}^{2}$ and $0.1 p b$ for a Higgs mass of $200 \mathrm{GeV} / \mathrm{c}^{2}$ at the Tevatron for a top quark mass of $30 \mathrm{GeV} / \mathrm{c}^{2}[41]$. Recent results from UA1 indicate that the top quark is heavier than this.

\subsection{The major collider experiments at Fermilab}

There are currently two large all purpose general detectors planned for Fermilab. They are the CDF detector (Figure 80) and the D0 detector (Figure (81). CDF has completed construction and has had a first data run of $\approx 30$ $n b^{-1}$ as of this writing. The CDF detector employs conventional calorimetry with scintillator readout in conjunction with a solenoidal magnetic field - The DO detector uses uranium liquid argon calorimetry and has no magnetic field in the electron sector. To help with the electron identification, it uses a transiton radiation detector. DO is currently in the construction phase and hopes to have its first data run in 1988 with the central part of its calorimeter in place. The first hermetic run is expected in 1989. 

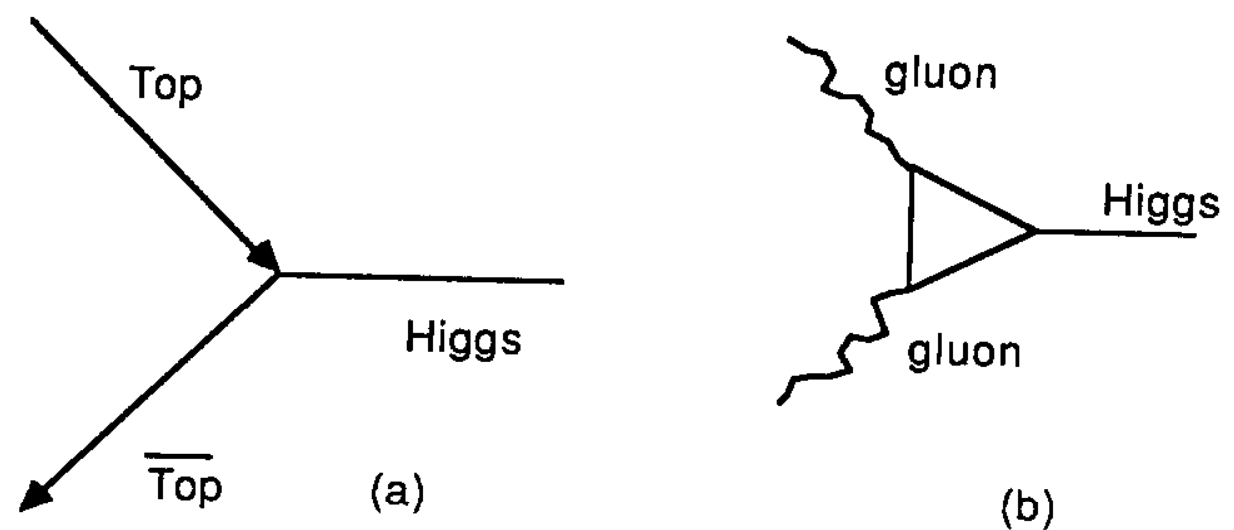

(b)

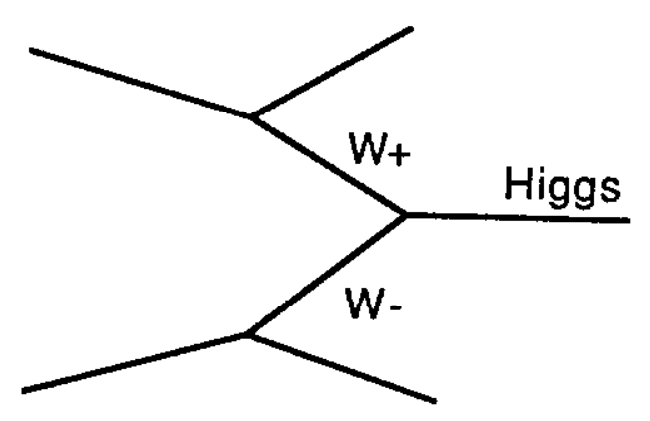

(c)

Figure 79: Feynman diagrams for Higgs Production in hadron collisions 


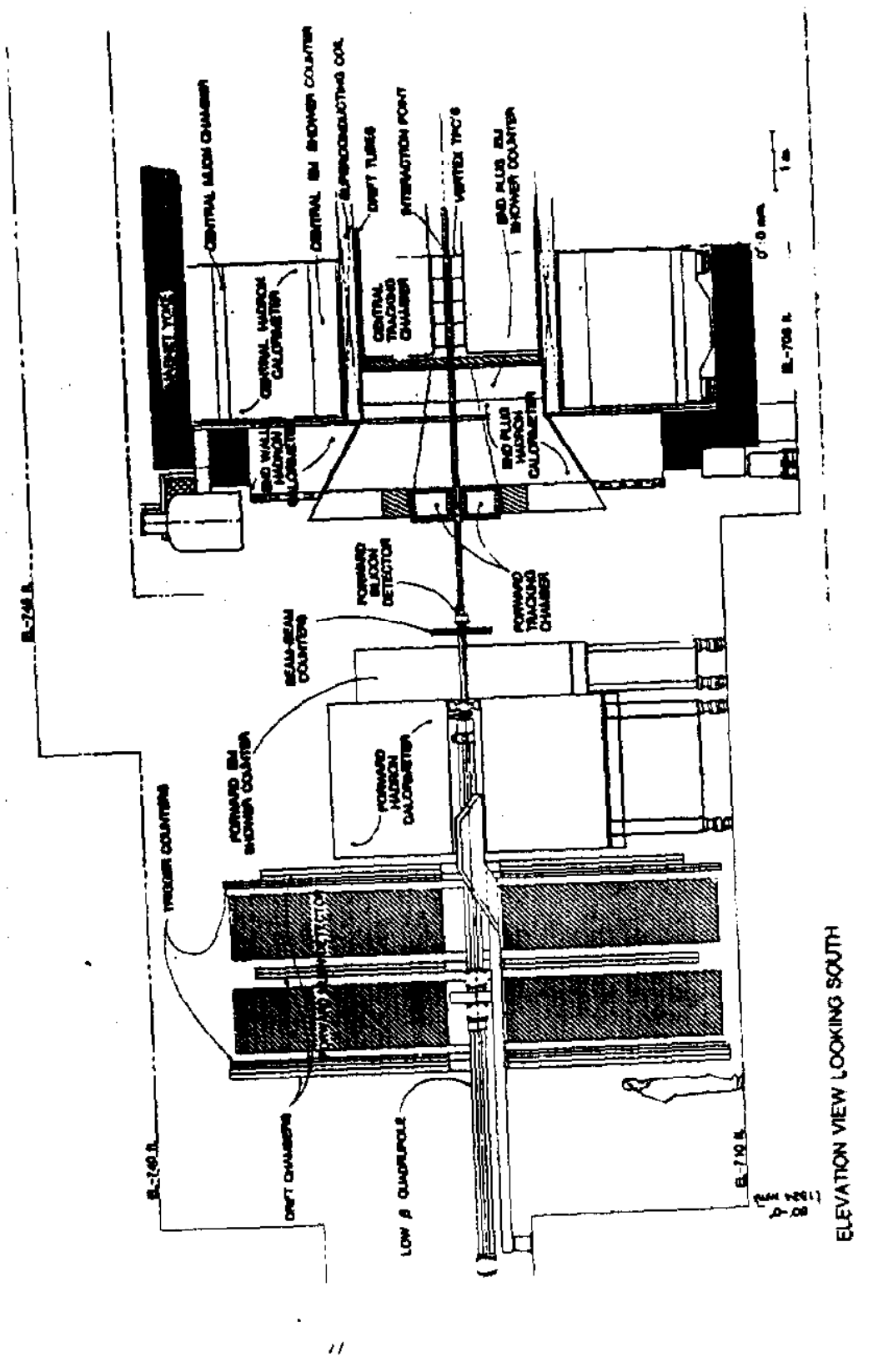

Figure 80: The CDF detector at Fermilab 


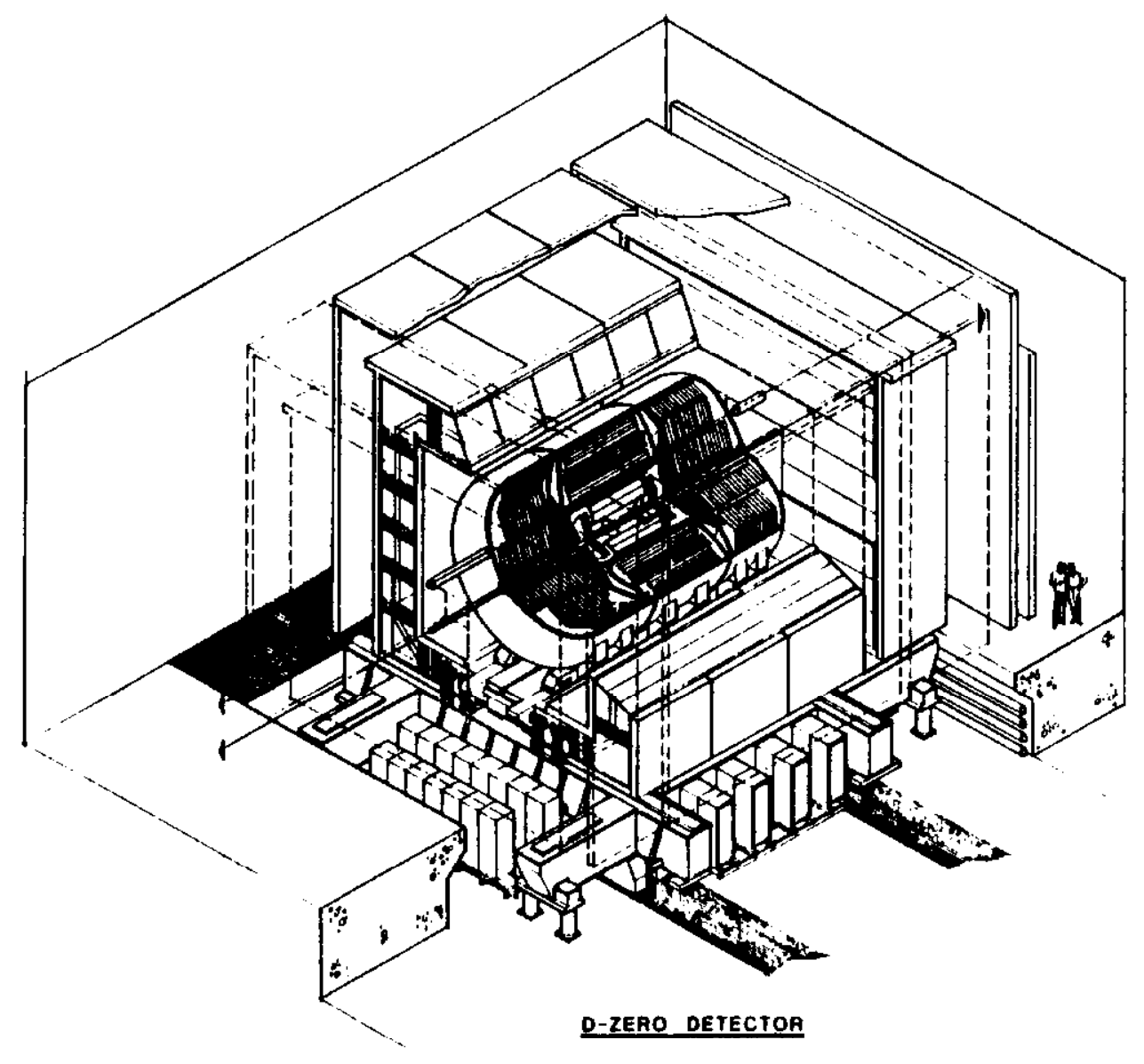

Figure 81: The D0 detector at Fermilab 


\subsection{Acknowledgements}

The author wishes to thank the organizers of the school, particularly A. Zepeda, J.Lucio and M.Moreno for their warm hospitality during the school and for their patience while this somewhat lengthy manuscript was being prepared. I also wish to thank Prof. Leon Lederman for his encouragement and his dedication to the cause of encouraging science in third world countries. 


\section{References}

[1] Hadronic interactions at highest energies.

By J.G. Rushbrooke,CERN-EP/85-178, Nov 1985. 59pp. Invited rapporteur's talk given at Int. Europhysics Conf. on High Energy Physics, Bari, Italy, 1985. Published in Bari Europhys.High Energy 1985:837

[2] Introduction to High Energy Physics, D.H.Perkins, Addison Wesley Publishing Company 1986. pp 124-130

[3] J.Velasco, UA4 collaboration, Contribution to the Bari Conference (1985)

[4] G.Chiefari, UA4 collaboration, Contribution to the Bari Conference (1985)

[5] M.Bozzo et al, Phys. Lett. 136B, 217 (1984)

[6] R. Castaldi and G.Sanguinetti, Elastic Scattering and Total Cross Section at Very High Energies Ann. Rev. Nucl. Part Sci. 1985, 351-395

[7] J. Dias de Deus, P.Kroll, J.Phys. G. 9, L81 (1983)

[8] U. Amaldi et al., Phys.Lett. 66B, 390 (1977).

[9] T.T. Wu and C.N.Yang, Phys. Rev. 137B, 708 (1965);

N.Byers and C.N.Yang, Phys. Rev. 142, 976 (1966);

T.T.Chou and C.N.Yang, Phys. Rev. 170, 1591 (1968);

L.Durand and R.Lipes, Phys.Rev.Lett. 20, 637 (1968)

[10] F.Hayot and U.Sukhatme, Phys.Rev. D10, 2183(1974);

T.T.Chou and C.N. Yang, Phys. Rev D19, 3268 (1979);

H.Cheng and T.T.Wu, Phys. Rev. Lett. 24,1456(1970)

[11] C.Bourrely, J.Soffer and T.T. Wu, Phys.Rev. D19,3249 (1979);Nucl. Phys. B247, 15 (1984) 
[12] A.B.Kaidalov, Phys.Lett. 116B, 459 (1982);

A.B.Kaidalov and K.A.Ter-Martirosyan, Phys.Lett 117B,247(1982); +references therein;

A.Capella, J.Tran Than Van, Z.Phys. C 18, 85 (1983) and references therein;

A.Capella, J. Kaplan, J.Tran Than Van, Nucl. Phys. 105B, 333 (1976);

C.Pajares, A.Varias, and P.Yepes, Z.Phys. C19, 89, (1983)

[13] L.V.Gribov, E.M.Levin and M.G.Ryskin, Phys.Rep 100,1, (1983) and references therin.

[14] A.Donnachie and P.V.Landshoff, Nucl.Phys. B244,322, (1984).

[15] R.G.Roberts and D.P.Roy, Nucl.Phys. B77,240 (1974).

[16] D.Amati et al., Nuovo Cimento. 26,896 (1962);

G.Fox, CALT68-43

[17] R.Hwa Phys.Rev.Lett. 26,1143 (1971);

M.Jacob and R.Slansky, Phys.Rev. D5, 1847 (1972)

[18] J.Gaudaen, Proceedings of the Bari Conference. (1985)

[19] A.Wroblewski, Multiplicity Distributions in $p p$ collisions, Acta Physica Polonica B4,857(1973)

[20] Z.Koba,H.B.Nielsen,P.Olesen, Nucl Phys. B40 (1972)

[21] P.Slattery, Phys Rev D7, 2073 (1973).

[22] F.T.Dao et al, Phys. Lett. 45B, 513(1973).

[23] A.Breakstone et al, Phys. Rev D30, 528 (1974)

[24] G.J.Alner et al, Phys. Lett. 138B, 304 (1984).

[25] G.J.Alner et al, Phys. Lett. 160B, 199 (1984). 
[26] M.Jacob, Proc. SLAC Topical Conference 27-29 July 1983.;

G.Pancheri, Y.Srivastava and M.Pallotta

Phys.Lett. 151B, 453 (1985);

G.Fowler, R.Weiner and G.Wilk, Univ. of Marburg Preprint (1985);

T.Gaisser,F.Halzen and A.Martin

University of Delaware preprint DTP/85/16;

S.Rudaz and P.Valin Phys. Rev D 34, 2025(1986);

C.Hayot and G.Sterman, Phys.Lett. 121B, 419 (1983);

D.Levy, Nucl.Phys. B59,583 (1973);

H.Navelet and R. Peschanski, Saclay preprint SphT/85/102

[27] A.Giovannini, Nuov. Cimento, 15A, 543 (1973);

W.J.Knox, Phys.Rev. D10, 65 (1974);

A.Giovannini et al Nuov. Cim 24A, 421 (1974);

P.Carruthers and C. Shih, Phys.Lett. 127B, 242 (1983);

P.Carruthers and C.Shih, Phys.Lett. 137B, 4215 (1984)

[28] L.Mclerran and Svetitsky, Phys. Lett 98B(1981) 195

[29] O.W. Greenberg and D.Zwanziger, Phys. Rev 150, 1177 (1966);

H.J.Lipkin, Phys. Lett 45B, 267 (1973);

Y.Nambu in Preludes in theoretical physics in honor of V.F.Weisskopf, edited by A. de Shalit, H.Feshbach and L. van Hove, North Holland, Amsterdam, Wiley, New York, 1966 p133.

[30] Y.Nambu in Preludes in theoretical physics in honor of V.F.Weisskopf, edited by A. de Shalit, H.Feshbach and L. van Hove, North Holland, Amsterdam, Wiley, New York, 1966 p133.;

M.Gell-Mann, Acta Phys Austriaca Suppl IV 733 (1972);

W.A.Bardeen, H.Fritsch and M.Gell-Mann in Scale and Conformal Symmetry in hadron physics, Ed. R.Gatto, Wiley, New York (1973) p139

[31] H.D.Politzer, Phys. Rev Lett. 30, 1346, (1973);

D.J.Gross and F.Wilczek, Phys. Rev. Lett. 30, 1343 (1973);

G. 'tHooft, Phys.Lett. 61B, 455 (1973);62B,444 (1973);

H.D.Politzer, Phys.Rep. 14C, 129 (1974) 
[32] J.D.Bjorken, Phys. Rev 163, 1767 (1967)

[33] see J.I. Friedman and H.W.Kendall

Ann. Rev. Nucl. Sci, 22, 203(1972) for a summary of the SLAC-MIT experiments.

[34] R.P.Feynman, Phys. Rev. Lett 23, 1415 (1969)

[35] S.D.Drell and T.M.Yan, Phys. Rev. Lett, 25316 (1970)

[36] R.D.Field and R.P.Feynman, Phys. Rev D15, 2590 (1977)

[37] Gauge theories of the strong, weak and electromagnetic interactions, Chris Quigg, Benjamin/Cummings publishing Company, Chapters 7,8

[38] K.F.Weizsacker Z.Phys. 88, 612 (1934);

E.J.Williams kgl. Danske Vid. Selsk. Mat. Fys. Medd. 13, 14 (1935)

[39] G.Altarelli, and G.Parisi, Nucl.Phys. B126, 298(1977)

[40] B.L.Combridge et al, Phys. Lett 70 B, 234 (1977)

[41] Estia Eichten, Fermilab-Conf-85/178-T (May 1986)

[42] H. Abramowicz et al (CDHS collaboration) Z.Phys. C13, 199(1982);

H. Abramowicz et al, Z.Phys C17 283 (1983)

[43] E. Eichten, I. Hinchliffe, K.Lane, and C. Quigg, Rev. Mod. Phys. 56, 579 (1984); and errata Fermilab-Pub-86/75-T (1986)

[44] M.Gluck, E.Hoffmann and E.Reya, Z.Phys. C13, 119 (1982).

[45] M. Banner et al, Phys. Lett. 118B , (1982) 203.

[46] G.Arnison et al, Phys. Lett. 123B (1983) 115.

[47] Hadronic jet production at the Cern $s \bar{p} p s$ collider. By UA1 Collaboration (G. Arnison, et al.), Phys.Lett.132B:214,1983.

[48] Jet fragmentation into charged particles at the Cern $s \bar{p} p s$ collider. By UA1 Collaboration (G. Arnison, et al.), Phys.Lett.132B:223,1983. 
[49] Angular distributions and structure functions from two jet events at the Cern $s \bar{p} p s$ collider. By UA1 Collaboration (G. Arnison, et al.), Phys.Lett.136B:294,1984.

[50] Comparison of three jet and two jet cross-sections in $\bar{p} p$ collisions at the Cern $s \bar{p} p s$ collider. By UA1 Collaboration (G. Arnison, et al.), Phys.Lett.158B:494,1985.

[51] Measurement of the inclusive jet cross-section at the Cern $\bar{p} p$ collider. By UA1 Collaboration (G. Arnison, et al.), Phys.Lett.B172:461,1986.

[52] Analysis of the fragmentation properties of quark and gluon jets at the Cern SPS $\bar{p} p$ collider. By UA1 Collaboration (G. Arnison, et al.), Nucl.Phys.B276:253,1986.

[53] Angular distributions for high mass jet pairs and a limit on the energy scale of compositeness for quarks from the Cern $\bar{p} p$ collider. By UA1 Collaboration (G. Arnison, et al.), Phys.Lett.177B:244,1986.

[54] Observation of very large transverse momentum jets at the Cern $s \vec{p} p s$ collider By UA2 Collaboration

(M. Banner, et al.), Phys.Lett.118B:203,1982.

[55] Measurement of production and properties of jets at the Cern $\bar{p} p$ collider. By UA2 Collaboration (P. Bagnaia, et al.), Z.Phys.C20:117,1983.

[56] Measurement of very large transverse momentum jet production at the Cern $\bar{p} p$ collider. By UA2 Collaboration (P. Bagnaia, et al.), Phys.Lett.138B:430,1984.

[57] Measurement of jet fragmentation properties at the Cern $\bar{p} p$ collider. By UA2 Collaboration (P. Bagnaia, et al.), Phys.Lett.144B:291,1984.

[58] Measurement of jet production properties at the Cern $\bar{p} p$ collider. By UA2 Collaboration (P. Bagnaia, et al.), Phys.Lett.144B:283,1984.

[59] Measurement of the $\sqrt{s}$ dependence of jet production at the Cern $\bar{p} p$ collider.

By UA2 Collaboration (J.A. Appel, et al.), Phys.Lett.160B:349,1985. 
[60] Experimental study of the emergence of two jet dominance in $\bar{p} p$ collisions at $\sqrt{(s)}=630 \mathrm{GeV}$. By UA2 Collaboration (J.A. Appel, et al.), Phys.Lett.165B:441,1985.

[61] A study of three jet events at the Cern $\bar{p} p$ collider. By UA2 Collaboration (J.A. Appel, et al.), Z.Phys.C30:341,1986.

[62] W.G.Scott, (UA1) . Presented at the International symposium on Physics of $\bar{p} p$ collisions, University of Tsukuba, KEK, March 13-15 (1985))

[63] F.Berends et al., Phys. Lett. 103B (1981) 124;

T.Gottschalk and D.Sivers, Phys. Rev D21 (1980) 102.

[64] E.Eichten, K.Lane and M. Peskin Phys. Rev. Lett. 50, 811 (1983).

[65] S.L.Glashow, Nucl. Phys. 22, 579 (1961)

[66] A.Salam, Elementary Particle theory, Relativistic Groups and analyticity (8th Nobel Symposium) edited by N.Svartholm, Almqvist and Wiksell, Stockholm, 1968, p.367

[67] S.Weinberg, Phys.Rev.Lett. 19, 1264(1967)

[68] G. 't Hooft, Nucl.Phys. B33, 173 (1971);B35, 167 (1971)

[69] G. 't Hooft and M. Veltman, Nucl. Phys. B44, 189 (1972); B50, 318 (1972).

[70] B.W.Lee and J. Zinn-Justin, Phys.Rev. D5, 3121,3137,3155 (1972); D7, 1049 (1973)

[71] F.J.Hasert et al, Phys. Lett. 46B, 121,138 (1973); Nucl. Phys. B73, 1 (1974)

[72] The following references give a partial list of experiments that tested the standard model outside the collider.

SLAC experiments in:

C.Y.Prescott et al, Phys. Lett. 77B, 347 (1978); 84B, 524 (1979); 
Atomic Physics experiments reviewed in:

E.D.Commins and P.H.Bucksbaum, Ann. Rev. Nucl.Part.Sci. 30, 1 (1980);

Neutrino interactions in:

H.Abramowicz et al. (CDHS collaboration) Phys. Rev. Lett 57, 298 ,(1986);

A.M.Cnops et al Phys. Rev. Lett 41, 357 (1978);

R.Blair (CCFRR)et al. Neutrino 81 proceedings Vol 1311 ;

[73] D.B.Cline, C.Rubbia, P.McIntyre, Neutrino 76: 683;

D.B.Cline, C.Rubbia, S. van der Meer, Sci. Am. 246, 38 (March, 1982)

[74] Stochastic Cooling theory and devices, S.van der Meer, (1978), proceedings, High Luminosity $\bar{p} p$ collisions, Berkeley $78 \mathrm{pp}$ 73-77

[75] UA1 W discovery was reported in :

G.Arnison et al, Phys. Lett. 122B, 103 (1983); ibid 126B, 398 (1983); ibid., 129B, 273 (1983);

UA2 $\mathrm{W}$ discovery was reported in:

M.Banner et al, Phys.Lett. 122B, 476 (1983); and P.Bagnaia et al., Phys. Lett. 129B, 130 (1983).

[76] Einhorn and Ellis, Phys. Rev. D12(1975) 2007.

[77] R.E.Shrock and L.L.Wang Phys.Rev. Lett. 411692 (1978).

[78] G.Altarelli, R.K.Ellis and G.Martinelli, Z.Phys.C27, 617 (1985)

[79] G.Altarelli, R.K.Ellis, M.Greco, and G.Martinelli Nucl. Phys. B., 246, $12(1984)$

[80] S.Geer and W.J. Stirling Phys. Lett. B 152373 (1985)

[81] Experimental observation of isolated large transverse energy electrons with associated missing energy at $\sqrt{s}=540-\mathrm{GeV}$. G. Arnison, et al.,Phys.Lett.122B:103,1983. 
[82] Experimental observation of lepton pairs of invariant mass around $95 \mathrm{GeV} / \mathrm{c}^{2}$ at the CERN $s \bar{p} p s$ collider.

G. Arnison, et al. Phys.Lett.126B:398,1983.

[83] Further evidence for charged intermediate vector bosons at the $s \bar{p} p s$ collider. G. Arnison, et al.Phys.Lett.129B:273,1983.

[84] Observation of the muonic decay of the charged intermediate vector boson. G. Arnison, et al.Phys.Lett.134B:469,1984.

[85] Observation of muonic Z0 decay at the $\bar{p} p$ collider. G. Arnison, et al.Published in Phys.Lett.147B:241,1984.

[86] W production properties at the CERN $s \bar{p} p s$ collider. G. Arnison, et al.Published in Nuovo Cim.Lett.44:1,1985.

[87] Intermediate vector boson properties at the CERN $s \bar{p} p s$ collider. G. Arnison, et al. Published in Phys.Lett.166B:484,1986.

[88] Events with large missing transverse energy at the CERN collider. Search for decays of $W^{ \pm}$into heavy leptons and of $Z^{0}$ into noninteracting particles. C. Albajar, et al. CERN-EP/86-82, Submitted to Phys. Lett. B

[89] Events with large missing transverse energy at the CERN collider: $W \rightarrow \nu_{\tau}$ decay and test of $\tau \mu e$ universality at $Q^{2}=M_{W}^{2}$. C. Albajar, et al., CERN-EP/86-81,Submitted to Phys. Lett. B

[90] Observation of single isolated electrons of high transverse momentum in events with missing transverse energy at the CERN $\bar{p} p$ collider. M. Banner, et al.,Phys.Lett.122B:476,1983.

[91] Evidence for $Z^{0} \rightarrow e^{+} e^{-}$at the CERN $\bar{p} p$ collider. P. Bagnaia, et al.,Phys.Lett.129B:130,1983.

[92] Measurement of $W^{ \pm}$and $Z^{0}$ properties at the CERN $\bar{p} p$ collider. J.A. Appel, et al.,Z.Phys.C30:1,1986.

[93] Search for decays of the $W^{ \pm}$and $Z$ bosons into $q \bar{q}$ pairs. R. Ansari, et al. CERN-EP/87-04, Submitted to Phys. Lett. B 
[94] Measurement of the standard model parameters from a study of W and $\mathrm{Z}$ bosons. R. Ansari, et al., CERN-EP/87-05,Submitted to Phys. Lett. B

[95] W.J.Marciano and A. Sirlin, Phys. ReV. D29 (1984)945.

[96] E. Locci, Invited talk give at the 8th European Symposium on Nucleonantinucleon interactions, Thessaloniki, 1986. CERN-EP/86-159.

[97] J.V.Allaby et al. (CHARM collab.) CERN-EP/86-94 (1986) Submitted to Phys.Lett.B;

Talks given at the 12th Int. Conf. on Neutrino Physics and Astrophysics, Sendai, Japan (1986);

by C.Guyot (CDHS collab.) , F.Merritt (CCFR Collab.) and R.Brock (FMMF Collab.)

[98] N.G.Deshpande et al,Phys. Rev. Lett. 54, 16, (1985) 1757.

[99] S.Geer, Proceedings of the XXIII International Conference on High Energy Physics, Berkeley, California, 1986.

[100] E.Duchovni, Proceedings of the XXIII International Conference on High Energy Physics, Berkeley, California, 1986.

[101] G.Arnison et al., Phys.Lett. 147B, 493 (1984).

[102] Z.Kunst et al.,Phys. Rev. D21, 733 (1980).

[103] Beauty Production at the CERN Proton-Antiproton Collider (Paper I), C. Albajar et al, CERN-EP/86-208 Submitted to Phys. Lett. B

[104] Search for $B^{0}-\overline{B^{0}}$ Oscillations at the CERN Proton-Antiproton Collider (Paper II), C.Albajar et al., CERN EP/86-209, Submitted to Phys. Lett. B

[105] F.Paige and S.D.Protopopescu, ISAJET Monte Carlo, BNL 38034 (1986).

[106] H. Schröder (ARGUS), DESY Seminar, 24 February 1987. 
[107] A Search for the Top in the UA1 Muon Plus Jet Data, "Two B's (and a gluon)

[108] The feasibility of observing $\eta_{t}$ to two photons at the CERN $\bar{p} p$ collider. S.Geer, R.Raja Phys. Lett. 150B(1985)223.

[109] R. Wigmans, CERN/EF 86-18, CERN/EP 86-141 (1986)

[110] R.Raja, D0 Note 461 (1986)

[111] J.Wess and B. Zumino, Phys. Lett. 49B (1974)52

[112] Experimental observation of events with large missing transverse energy accompanied by a jet or a photon(s) in $\bar{p} p$ collisions at $\sqrt{(s)}=$ $540 \mathrm{GeV}$

By UA1 Collaboration,Phys.Lett.139B:115,1984.

H.Behrend et al., CELLO collaboration, Phys.Lett. 123B, 127 (1983) for photino limits

B.Alper et al, Phys.Lett. 46B,265(1973); and D.Cutts et al Phys.Rev.Lett. 41, 363 (1978) for charged R Hadron limits.

R.Gustafson et al, Phys.Rev. Lett. 37,474(1976) for neutral R Hadron limits.

R.C.Ball et al., Phys. Rev. Lett. 53, 1314 (1984); F. Bergsma et al., CHARM collaboration, Phys. Lett. 121B, 429 (1983) for beam dump limits on gluino mass and lifetime.

E. Fernandez et al., MAC collaboration Phys. Rev. Lett 54, 1118 (1985) for longlived fractionally charged particle limits which can be interpretted as squark limits.

[113] A.Honma, Proceedings of the XXIII International Conference on High Energy Physics, Berkeley, California, 1986.

[114] K.Freese, and D.Schramm, Nucl.Phys. B233, 167 (1984)

[115] H. Goldberg, Phys. Rev. Lett. 50, 1419 (1983)

[116] N. Cabibbo, G.Farrar, and L.Maiani, Phys. Lett. 105B. 155 (1981). 
[117] S. Weinberg, Phys. Rev. D19, 1277 (1979)

[118] L.Susskind, Phys. Rev. D20, 2619 (1979)

[119] E. Farhi, L.Susskind, Phys. Rev. D20 , 3404 (1979)

S. Dimopoulos, Nucl. Phys. B166, 69 (1980).

M. Peskin Nucl. Phys. B 175, 197 (1980).

J. Preskill, Nucl. Phys. B 177, 21 (1981).

S. Dimopoulos, S. Raby and G.L. Kane, Nucl. Phys. B182, 77 (1981). 